\title{
The trace field of hyperbolic gluings
}

\author{
Inauguraldissertation \\ der Philosophisch-naturwissenschaftlichen Fakultät \\ der Universität Bern
}

vorgelegt von

Olivier Mila

von Huttwil

\author{
Leiter der Arbeit: \\ Prof. Dr. Vincent Emery \\ Mathematisches Institut der Universität Bern
}

Originaldokument gespeichert auf dem Webserver der Universitätsbibliothek Bern.

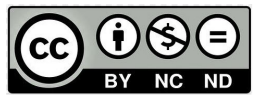

Dieses Werk ist unter einem Creative Commons Namensnennung-Keine kommerzielle Nutzung-Keine Bearbeitung 2.5 Schweiz Lizenzvertrag lizenziert.

Um die Lizenz anzusehen, gehen Sie bitte zu

http://creativecommons.org/licenses/by-nc-nd/2.5/ch/

oder schicken Sie einen Brief an Creative Commons, 171 Second Street,

Suite 300, San Francisco, California 94105, USA. 


\section{Urheberrechtlicher Hinweis}

Dieses Dokument steht unter einer Lizenz der Creative Commons Namensnennung-Keine kommerzielle Nutzung-Keine Bearbeitung 2.5 Schweiz. http://creativecommons.org/licenses/by-nc-nd/2.5/ch/

\section{Sie dürfen:}

dieses Werk vervielfältigen, verbreiten und öffentlich zugänglich machen

\section{Zu den folgenden Bedingungen:}

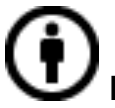

Namensnennung. Sie müssen den Namen des Autors/Rechteinhabers in der von ihm festgelegten Weise nennen (wodurch aber nicht der Eindruck entstehen darf, Sie oder die Nutzung des Werkes durch Sie würden entlohnt).

Keine kommerzielle Nutzung. Dieses Werk darf nicht für kommerzielle Zwecke verwendet werden.

\section{$\Theta$}

Keine Bearbeitung. Dieses Werk darf nicht bearbeitet oder in anderer Weise verändert werden.

Im Falle einer Verbreitung müssen Sie anderen die Lizenzbedingungen, unter welche dieses Werk fällt, mitteilen.

Jede der vorgenannten Bedingungen kann aufgehoben werden, sofern Sie die Einwilligung des Rechteinhabers dazu erhalten.

Diese Lizenz lässt die Urheberpersönlichkeitsrechte nach Schweizer Recht unberührt.

Eine ausführliche Fassung des Lizenzvertrags befindet sich unter http://creativecommons.org/licenses/by-nc-nd/2.5/ch/legalcode.de 


\title{
The trace field of hyperbolic gluings
}

\author{
Inauguraldissertation \\ der Philosophisch-naturwissenschaftlichen Fakultät \\ der Universität Bern
}

vorgelegt von

\section{Olivier Mila}

von Huttwil

\author{
Leiter der Arbeit: \\ Prof. Dr. Vincent Emery \\ Mathematisches Institut der Universität Bern
}

Von der Philosophisch-naturwissenschaftlichen Fakultät angenommen.

Bern, 23. August 2019

Der Dekan:

Prof. Dr. Zoltán Balogh 



\section{Contents}

$\begin{array}{lll}1 & \text { Introduction } & 7\end{array}$

2 Background $\quad 11$

2.1 Linear algebraic groups . . . . . . . . . . . . . . . 11

2.1.1 Affine algebraic varieties . . . . . . . . . . . . 11

2.1.2 Fields of definition . . . . . . . . . . . . . . . . 12

2.1.3 Linear algebraic groups . . . . . . . . . . . . . . 14

2.1.4 Dimension, tangent space, adjoint representation . . . 16

2.1.5 Classical groups . . . . . . . . . . . . . . . 18

2.1.6 Automorphisms of $\mathbf{P O}_{f} \ldots \ldots \ldots . \ldots 21$

2.2 Hyperbolic geometry . . . . . . . . . . . . . . . . . . 23

2.2.1 Hyperbolic manifolds . . . . . . . . . . . . . . . 23

2.2.2 Hyperplanes and boundary . . . . . . . . . . . . . 25

2.2.3 Commensurability and torsion . . . . . . . . . . 28

2.2.4 Subgroup separability . . . . . . . . . . . . . . 28

2.2.5 The hyperbolic models $\mathbf{H}_{f} \ldots \ldots \ldots$. . . . . . . . 29

2.3 Arithmetic hyperbolic manifolds . . . . . . . . . . . . . . . 30

2.3.1 Definition of arithmetic lattices . . . . . . . . . 30

2.3.2 Examples . . . . . . . . . . . . . . . 33

2.3.3 Hypersurfaces in arithmetic manifolds . . . . . . . . . . 34

2.3.4 Subgroup separability in arithmetic lattices . . . . . . . . 36

$\begin{array}{llr}3 & \text { Trace Fields } & 39\end{array}$

3.1 Trace fields and ambient groups . . . . . . . . . . . . . 39

3.1.1 Definition for Zariski-dense subgroups . . . . . . . . . . . 39

3.1.2 Definition for lattices . . . . . . . . . . . . . . . . 41

3.1.3 Definition for manifolds with boundary . . . . . . . . . 43

3.2 Manifolds with hypersurfaces . . . . . . . . . . . . . . . 43

3.2.1 Rational reflections . . . . . . . . . . . . . . 44

3.2 .2 Manifolds of type I . . . . . . . . . . . . . . . . 47

3.3 Computation in the arithmetic case . . . . . . . . . . . . . . 48 
4 Gluings $\quad 51$

4.1 General hyperbolic gluings . . . . . . . . . . . . . . 51

4.1.1 Interbreeding and closing up . . . . . . . . . . 51

4.1 .2 Arithmetic pieces . . . . . . . . . . . . . 53

4.2 Nonarithmetic gluings . . . . . . . . . . . . . . . . 55

4.2.1 Gromov and Piatetski-Shapiro's construction . . . . . . 55

4.2 .2 Generalizations . . . . . . . . . . . . 56

4.3 Trace fields and ambient groups of gluings . . . . . . . . . . . 57

4.3.1 Field of definition of gluing isometries . . . . . . . . . 57

4.3.2 The trace field of gluings . . . . . . . . . . . . . . . 59

4.4 Corollaries and computations . . . . . . . . . . . . . . 62

4.4.1 Gluing and quadratic extensions . . . . . . . . . . . . . 62

4.4 .2 Doubling and trace field . . . . . . . . . . . . 63

4.4 .3 Odd dimensional closing up . . . . . . . . . . . . 64

4.4.4 Gluings of arithmetic pieces . . . . . . . . . . . . 64

4.5 Gluing commensurable manifolds . . . . . . . . . . . . . 66

4.5.1 Gromov-Piatetski-Shapiro revisited . . . . . . . . . . 66

4.5.2 Closing up arithmetic pieces . . . . . . . . . . 68

$\begin{array}{lll}5 & \text { Pseudo-arithmeticity and Volumes } & \mathbf{7 3}\end{array}$

5.1 Pseudo-arithmeticity and gluings . . . . . . . . . . . . 73

5.1 .1 Pseudo-arithmeticity . . . . . . . . . . . . . . 73

5.1 .2 Gluing of arithmetic pieces . . . . . . . . . . . 76

5.2 Homology . . . . . . . . . . . . . . . . 76

5.2 .1 Classical homology . . . . . . . . . . . . . 76

5.2 .2 Simplicial homology . . . . . . . . . . . . . 78

5.2 .3 Relative homology . . . . . . . . . . . . . . . . 78

5.2.4 Relative homology of lattices . . . . . . . . . . . . 79

5.2.5 Relative homology and volumes . . . . . . . . . . . 80

5.3 Volumes of pseudo-arithmetic manifolds . . . . . . . . . . . 81

5.3.1 Homology of ambient groups . . . . . . . . . . . . . 81

5.3 .2 Lemmas . . . . . . . . . . . . . . . . . . . 82

5.3 .3 Proofs ....................... 85 


\section{Chapter $1 \mid$ Introduction}

Introduced in 1987 by M. Gromov and I. Piatetski-Shapiro in [GPS87], the gluing construction for hyperbolic manifolds was the first method to produce nonarithmetic manifolds in any dimension $n$, and remains (along with its generalizations) the only one as of today. Prior to that, nonarithmetic examples were obtained using reflection groups, first in dimension $n=3$ by $\mathrm{V}$. Makarov [Ma66], then in dimension $n=4,5$ by E. Vinberg [Vi67]. However, it is known that discrete reflection groups of finite covolume (resp. cocompact) do not exist in dimensions $n \geq 996$ (resp. $n \geq 30$ ), see [Pr86] (resp. [Vi81]).

Roughly, the idea of the gluing construction is to start with arithmetic manifolds that are different enough (i.e., non-commensurable) but which contain a common embedded hypersurface. By cutting these manifolds open at the hypersurface, one obtains "pieces" of manifolds that have isometric boundary components. These pieces can then be glued together along their boundary to form a "hybrid" manifold, which turns out to be nonarithmetic (see Figure 1.1). This construction was generalized to more complicated gluings, notably by J. Raimbault [Ra13], T. Gelander and A. Levit [GL14] and M. Belolipetsky and S. Thomson [BT11] (following an idea of I. Agol [Ag06]). These have important consequences such as the superexponential growth of the number of nonarithmetic manifolds of volume less than $v$, as $v \rightarrow \infty$, or the existence of hyperbolic manifolds with arbitrarily small systole.

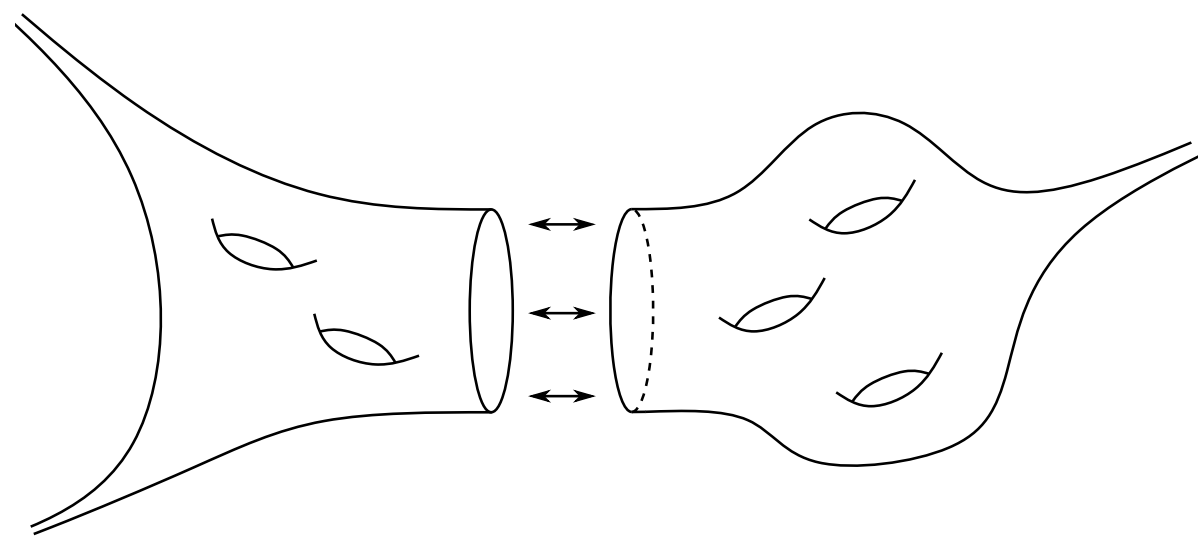

Figure 1.1: Schematic picture of hyperbolic gluing. 
Note that the existence of nonarithmetic lattices is in sharp contrast with the situation in (almost) all other locally symmetric spaces (in particular those of rank $\geq 2$ ), where Margulis' arithmeticity theorem implies that all the corresponding manifolds are arithmetic.

As their name suggests, arithmetic manifolds (or equivalently, arithmetic lattices) are closely related to number theoretic objects: they are constructed using the ring of algebraic integers of a number field (the field of definition) and an algebraic group with certain properties. In his 1971 paper [Vi71], Vinberg introduced a generalization of these invariants (called the trace field and the ambient group, respectively) to arbitrary Zariski-dense subgroups of semisimple algebraic groups. For a lattice $\Gamma$, the trace field can be seen as the smallest field $k$ such that $\Gamma$ can be realized in $\mathbf{G}(k)$ for some algebraic group $\mathbf{G}$ (which is precisely the ambient group). Furthermore, Vinberg showed that these are commensurability invariants, and they thus give a useful way to check (non)arithmeticity of the corresponding manifolds.

The main result of this thesis concerns the trace fields and ambient groups of the gluing constructions described above. In this context, a central role is played by the gluing isometry (i.e., the one used to identify the boundary components of the manifolds). In Section 4.3, we prove that such a gluing isometry naturally admits a field of definition, and in Theorem 4.7 we show that it coincides with the trace field of the corresponding gluing.

This result is valid for all gluings (that is, involving not necessarily arithmetic pieces) and can be used to compute their trace field explicitly. In the case of the manifolds of Gromov and Piatetski-Shapiro, we show that (if the gluing isometries are the canonical ones, see Section 4.4.4) the trace field is a quadratic extension of the field of definition of the original arithmetic manifolds (Corollary 4.14). In particular, this gives another proof that these gluings are nonarithmetic. We also obtain similar results for the various generalizations of this construction.

On a more theoretical side, we use Theorem 4.7 to deduce results about specific gluing constructions on general manifolds. In particular, we prove that:

- The trace field of a gluing always contains the trace field of the pieces, and each gluing step (that is, each identification of two hypersurfaces) increases the trace field by at most a quadratic extension (Theorem 4.10),

- The trace field of a piece coincides with the trace field of its double (Proposition 4.11),

- Closing up a piece (that is, gluing two of its isometric boundary components together, see Figure 1.2) does not change the trace field provided the dimension is odd (Proposition 4.12).

Moreover, we construct even-dimensional pieces of arithmetic manifolds whose trace field increases when closing up (see Section 4.5). These provide explicit 
examples in arbitrary (even) dimension of nonarithmetic manifolds having precisely the same volume as an arithmetic one.

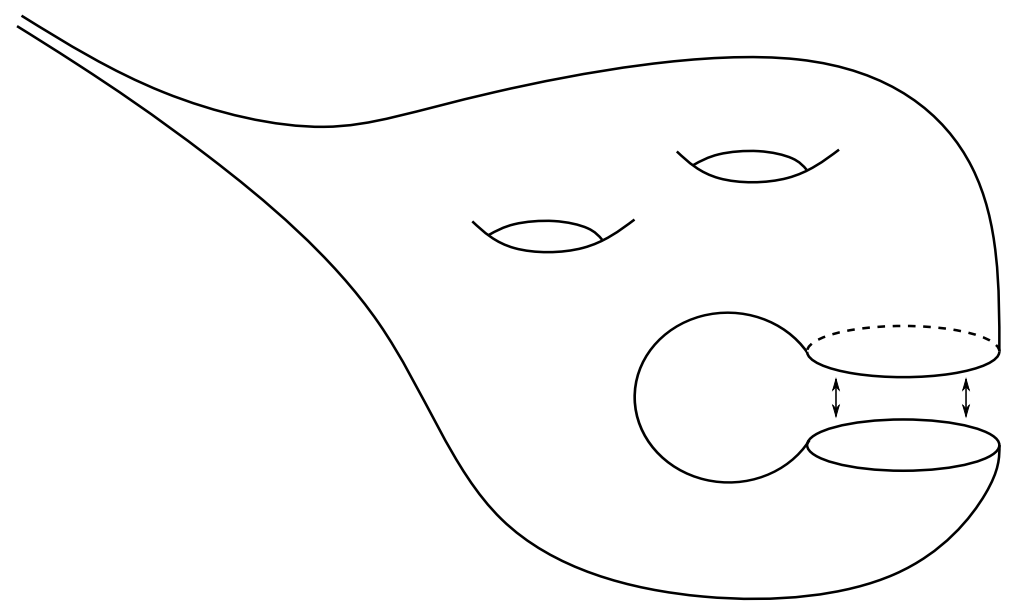

Figure 1.2: Closing up a hyperbolic manifold.

In the final part of this thesis (based on a joint work [EM18] with Vincent Emery), we introduce and motivate the notion of pseudo-arithmeticity using the theory developed in the previous chapters. Pseudo-arithmetic manifolds can be seen as a generalized version of gluings of pieces of arithmetic manifolds (see the remark after their definition in Section 5.1.1). Indeed, we first show (using Theorem 4.7 as the main tool) that all gluings of arithmetic pieces are pseudo-arithmetic (Theorem 5.1). Second, we prove (under an additional restriction on their ambient groups) that their volume is always a rational linear combination of volumes of arithmetic manifolds (Theorem 5.2).

This thesis is organized as follows. In Chapter 2 we introduce the necessary background needed for the rest of the text. Chapter 3 is devoted to the definition of the trace field and ambient groups following Vinberg, as well as some computations in the case of arithmetic manifolds and manifolds containing hypersurfaces. All the results in these two chapters are (more or less) standard; yet we have included some proofs when they are particularly nice, particularly simple or lacking a suitable reference.

Chapter 4 contains the main results of this thesis concerning gluings. After defining them in their most general form, we compute their trace field and derive the corollaries mentioned above. Finally Chapter 5 treats pseudoarithmeticity and its consequences, following [EM18].

\section{Acknowledgements.}

Uppermost on the thank you list is of course my advisor Vincent Emery. Accessible, supportive and generous are three of the numerous qualities he possesses, and thanks to his guidance and mathematical intuition he taught me a lot and made these four years very pleasant and interesting. Merci! 
I am also grateful to my academic family: my brother Ruben Bär and my uncles Scott Thomson and Plinio G. P. Murillo, for their friendship and the stimulating mathematical discussions we shared.

I warmly thank the two referees Jean Raimbault and Matthew Stover who had the courage of reading my thesis; their comments and suggestions helped me improve the manuscript substantially. I am also very grateful to the chairman Sebastian Baader, for his mathematical insight and for the nice backgammon sessions.

I do not forget all the members of the mathematical (and statistical) institute that brightened the countless days I spent in the office. In particular, I would like to thank Annina Iseli, Luca Studer, Lukas Lewark, Livio Liechti, Filip Misev and Alex Ramos for welcoming me into the arms of Alp22.

Of my friends, I wish to thank all. A special thought goes to those I met during my studies at EPFL, in informatics for letting me change to math, and in math for welcoming me even though I came from informatics. Thanks also to Charles Dalang, Deyan Poffet, Yannick Poffet and the Seahawk 4.

Penultimately, I wish to thank Martino Borello and Eva Bayer-Fluckiger for giving me the motivating opportunity to do research already during my Master thesis.

Finally, I thank my parents Eva and Frédéric and my brother Thibaud for their love and never ending encouragements. And of course, I thank Lucie Giesbrecht with all my heart for supporting me (both in the English and the French sense) during all these years and for her constant positivity, especially in the last months. I most probably could not have done it without her. 


\section{Chapter $2 \mid$ Background}

This chapter introduces the necessary background and notation for the rest of the thesis. The reader is assumed familiar with basic concepts of algebra and geometry. In particular, we will use without introduction elements of ring and field theory, Galois theory, quadratic forms and a little number theory on the algebra side, and concepts of topology, covering space theory and Riemannian geometry on the geometric side. This chapter contains only standard (well-known) material, where (as explained in the introduction) some proofs have been included either for aesthetic reasons or simply for a lack of suitable reference.

\section{$2.1 \mid$ Linear algebraic groups}

This section recalls the notions of algebraic geometry and algebraic groups we will need. Good references are Hartshorne [Ha77] as well as Borel [Bo91, Chapter AG] and Platonov and Rapinchuk [PR94, Chapter 2]. In this section $k$ is an arbitrary subfield of $\mathbb{C}$.

2.1.1 Affine algebraic varieties. We will start by defining the main concepts of algebraic geometry, namely affine algebraic varieties.

Definition 2.1.1. The $k$-Zariski topology on $\mathbb{C}^{n}$ is the topology where the closed sets are $\mathbb{C}^{n}$ and the empty set together with sets of the form

$$
V\left(\left\{f_{1}, \ldots, f_{r}\right\}\right)=\left\{x \in \mathbb{C}^{n} \mid f_{1}(x)=\cdots=f_{r}(x)=0\right\},
$$

for $r \in \mathbb{N}$ and $f_{i} \in k\left[x_{1}, \ldots, x_{n}\right]$. It is clear that we may replace the set $\left\{f_{1}, \ldots, f_{r}\right\}$ by the ideal $I \subset k\left[x_{1}, \ldots, x_{n}\right]$ generated by the $f_{i}$. An affine algebraic $k$-variety is a $k$-Zariski closed subset of $\mathbb{C}^{n}$ for some $n$. A morphism $\varphi: V \rightarrow W$ of $k$-varieties $V \subset \mathbb{C}^{m}, W \subset \mathbb{C}^{n}$ is a map $\varphi: V \rightarrow W$ which is induced by a polynomial map $k^{m} \rightarrow k^{n}$, i.e., such that there exist $f_{1}, \ldots, f_{n}$ in $k\left[x_{1}, \ldots, x_{m}\right]$ with $\varphi\left(x_{1}, \ldots, x_{m}\right)=\left(f_{1}\left(x_{1}, \ldots, x_{m}\right), \ldots, f_{n}\left(x_{1}, \ldots, x_{m}\right)\right)$. An isomorphism is a morphism with an inverse that is also a morphism. 
The coordinate ring $k[V]$ of an affine algebraic $k$-variety $V \subset \mathbb{C}^{n}$ is the ring of functions $V \rightarrow \mathbb{C}$ which are induced by polynomials in $k\left[x_{1}, \ldots, x_{n}\right]$. Thus a map $V \rightarrow W$ is a morphism if and only if it induces a map $k[W] \rightarrow k[V]$. An embedding is an injective morphism $V \rightarrow W$ such that the induced map $k[W] \rightarrow k[V]$ is surjective.

If $V$ is a $k$-variety and $K / k$ is a field extension (with $K \subset \mathbb{C}$ ), the base change of $V$ to $K$, denoted $V_{K}$, is the variety $V$ seen over the field $K$. Finally, the $K$-points of $V \subset \mathbb{C}^{n}$ are defined as $V(K)=V \cap K^{n}$.

Since all our varieties will be affine, we will often write $k$-variety or simply variety if the field is clear from the context.

Remark 2.1.2. Usually the term variety is reserved for irreducible $k$-closed sets, that is, those that cannot be written as a finite union of proper closed subsets. However, we will not (in general) assume this, and use the terms variety and $k$-closed set interchangeably.

If $I \subset k\left[x_{1}, \ldots, x_{n}\right]$ is an ideal, we let $\sqrt{I}=\left\{f \mid f^{r} \in I\right.$ for some $\left.r\right\}$ denote the radical of $I$. By Hilbert's Nullstellensatz, the polynomial functions in $k\left[x_{1}, \ldots, x_{n}\right]$ vanishing on a variety $V=V(I) \subset \mathbb{C}^{n}$ defined by the ideal $I$ are precisely the elements of $\sqrt{I}$. Thus if $I$ is radical (i.e., $I=\sqrt{I}$ ), we have a canonical isomorphism $k[V] \cong k\left[x_{1}, \ldots, x_{n}\right] / I$.

An affine algebraic $k$-variety $V$ is characterized by its algebra $k[V]$ in the following sense: two $k$-varieties $V$ and $W$ are isomorphic if and only if $k[V]$ and $k[W]$ are isomorphic as $k$-algebras. Furthermore, any finitely generated commutative $k$-algebra $A$ without nilpotents corresponds to a $k$-variety $V$ as follows: from finite generation we find a morphism $k\left[x_{1}, \ldots, x_{n}\right] \rightarrow A$, with kernel an ideal $I$. Then by definition $V=V(I) \subset \mathbb{C}^{n}$ has $k[V]=A$.

Definition 2.1.3. The product variety $V \times W$ of varieties $V$ and $W$ is the variety associated with the algebra $k[V] \otimes_{k} k[W]$. If $V=V(I) \subset \mathbb{C}^{m}$ with $I \subset k\left[x_{1}, \ldots, x_{m}\right]$ and $W=V(J) \subset \mathbb{C}^{n}$ with $J \subset k\left[y_{1}, \ldots, y_{n}\right]$, we have

$$
V \times W \cong V(I \cdot J) \subset \mathbb{C}^{m+n} .
$$

2.1.2 Fields of definition. We now define (minimal) fields of definition.

Definition 2.1.4. Let $V$ be a $\mathbb{C}$-variety. Then $V=V(I)$ for some ideal $I \subset \mathbb{C}\left[x_{1}, \ldots, x_{n}\right]$. If $I$ admits a generating set in $k\left[x_{1}, \ldots, x_{n}\right]$ for some field $k \subset \mathbb{C}$, we say that $V$ is defined over $k$. We then have that $V=W_{\mathbb{C}}$ for the $k$-variety $W$ defined by the ideal $J=I \cap k\left[x_{1}, \ldots, x_{n}\right]$. The smallest field such that this holds is called the field of definition of $V$ (see Remark 2.1.6 for comments about existence of such a field).

We can define a similar field for morphisms. Let $V$ and $W$ be two $k$-varieties, and let $\varphi: V_{\mathbb{C}} \rightarrow W_{\mathbb{C}}$ be a morphism between the base extensions to $\mathbb{C}$. Then $\varphi$ 
is defined over $K$ for some field extension $K / k$ contained in $\mathbb{C}$ if the subvariety $Z_{\varphi}=\{(x, f(x)) \mid x \in V\} \subset V \times W$ is defined over $K$. The field of definition of $\varphi$ is the field of definition of $Z_{\varphi}$, composed with $k$ (see next remark).

Remark 2.1.5. We will use the convention that a morphism $\varphi: V_{\mathbb{C}} \rightarrow W_{\mathbb{C}}$ is not defined over a smaller field than the base field of $V$ and $W$. More precisely, if $V$ and $W$ are $k$-varieties, the field of definition of $\varphi$ will always be assumed to contain $k$, even when the fields of definition of $V$ and $W$ are smaller.

Remark 2.1.6. The existence of a minimal field of definition (for affine varieties) was first proven by Weil. See [CGP15, Definition 1.1.6] and [EGA4, Section 4.8.11] (and perhaps [Ku85, Exercise 1.2.9]).

Let $k \subset \mathbb{C}$ be a subfield, and let $G$ be the group of $k$-automorphisms of $\mathbb{C}$. Observe that $G$ acts on $\mathbb{C}^{n}$, and thus on $\mathbb{C}\left[x_{1}, \ldots, x_{n}\right]$ by:

$$
\sigma\left(\sum a_{i} x^{i}\right)=\sum \sigma\left(a_{i}\right) x^{i}
$$

where the index $i$ ranges over $\mathbb{N}^{n}$ and $x^{i}=x_{1}^{i_{1}} \cdots x_{n}^{i_{n}}$. If we consider the polynomial $f \in \mathbb{C}\left[x_{1}, \ldots, x_{n}\right]$ as a function $f: \mathbb{C} \rightarrow \mathbb{C}$, this action rewrites as

$$
\sigma(f)=\sigma \circ f \circ \sigma^{-1} \text {. }
$$

It follows that if $V \subset \mathbb{C}^{n}$ is a variety with ideal $I \subset \mathbb{C}\left[x_{1}, \ldots, x_{n}\right]$ then $\sigma(V)$ is also a variety, corresponding to the ideal $\sigma(I) \subset \mathbb{C}\left[x_{1}, \ldots, x_{n}\right]$. The following lemma gives a criterion for deciding when a variety is defined over $k$.

Lemma 2.1. Let $V$ be a $\mathbb{C}$-variety. Then $V$ is defined over $k$ if and only if $V$ is fixed by $G$, that is, if $\sigma(V)=V$ for each $\sigma \in G$.

Proof. If $V$ is a $k$-variety, then its defining polynomials have coefficients in $k$. Thus they are fixed by $G$, and so is $V$. Conversely, suppose $V$ is fixed by $G$. Since $V$ is the zero locus of finitely many polynomials, $V$ is defined over a finitely generated extension $K / k$. By the classification of transcendental extensions, there are algebraically independent elements $t_{1}, \ldots, t_{n}$ such that $K$ is an algebraic extension of $\ell=k\left(t_{1}, \ldots, t_{n}\right)$, which we can assume to be Galois. Now let $\sigma \in \operatorname{Gal}(K / \ell)$ be an element of the Galois group. Then one can extend $\sigma$ to an automorphism $\sigma \in G$, which by hypothesis fixes $V$. Thus $V$ is fixed by $\operatorname{Gal}(K / \ell)$, and is therefore defined over $\ell$ by [Bo91, Theorem AG.14.4].

It remains to show that $V$ is defined over $k$. Let $I$ be the defining ideal of $V$; it is an $\ell$-vector subspace of $\ell\left[x_{1}, \ldots, x_{N}\right]$ for some $N$. Let $I_{\mathbb{C}}$ be the ideal in $\mathbb{C}\left[x_{1}, \ldots, x_{N}\right]$ generated by $I$; we have $I=I_{\mathbb{C}} \cap \ell\left[x_{1}, \ldots, x_{N}\right]$. Since $I_{\mathbb{C}}$ is the intersection of all maximal ideals containing it, and since $G$ fixes this collection, we see that $I$ is also fixed by $G$. Now it suffices to observe that the same proof of the proposition of $\S 14.2$ in [Bo91] works in our more general case (since for any $a \in \ell \backslash k$ there is a $k$-automorphism $\sigma$ of $\ell$ with $\sigma(a) \neq a$ ). Therefore $I$ is the $\ell$-span of some $k$-vector space $I_{k} \subset k\left[x_{1}, \ldots, x_{N}\right]$, and $V$ is defined over $k$. 
Corollary 2.2. Let $V, W$ be $k$-varieties and let $\Gamma \subset V(k)$ be a Zariski-dense subset of $V$. Let $\varphi: V_{\mathbb{C}} \rightarrow W_{\mathbb{C}}$ be a morphism such that $\varphi(\Gamma) \subset W(k)$. Then $\varphi$ is defined over $k$.

Proof. Let $\sigma \in G$ be a $k$-automorphism of $\mathbb{C}$. Then the map ${ }^{\sigma} \varphi=\sigma \circ \varphi \circ \sigma^{-1}$ is also a morphism $V_{\mathbb{C}} \rightarrow W_{\mathbb{C}}$ which agrees with $\varphi$ on $\Gamma$. If $W \subset \mathbb{C}^{N}$ for some $N$, the map $\varphi-{ }^{\sigma} \varphi: V \rightarrow \mathbb{C}^{N}$ is zero on $\Gamma$ and hence also on $V$ by Zariski-density. It follows that $\varphi={ }^{\sigma} \varphi$.

We now consider the graph $Z_{\varphi}=\{(x, \varphi(x)) \mid x \in V\} \subset V \times W$. Clearly

$$
\sigma\left(Z_{\varphi}\right)=Z_{(\sigma \varphi)}=Z_{\varphi}
$$

It follows that $Z_{\varphi}$ is fixed by all $k$-automorphisms of $\mathbb{C}$, whence defined over $k$ by the lemma. By definition, this means that $\varphi$ is defined over $k$.

\subsubsection{Linear algebraic groups.}

Definition 2.1.7. A linear (or affine) algebraic k-group $\mathbf{G}$ is an algebraic $k$-variety with a group structure, such that multiplication $\mathbf{G} \times \mathbf{G} \rightarrow \mathbf{G}$ and inverse $\mathbf{G} \rightarrow \mathbf{G}$ are morphisms of $k$-varieties. A morphism between linear algebraic groups is a morphism of varieties that is also a group homomorphism. The identity (or neutral) component $\mathbf{G}^{\circ}$ of $\mathbf{G}$ is the connected component of G containing 1 .

It can be shown that $\mathbf{G}^{\circ}$ is actually a closed subgroup of $\mathbf{G}$ of finite index (see [Br10]). Since all algebraic groups considered in this thesis will be linear, we will usually only write algebraic groups, or $k$-groups if we wish to specify the field.

Example 2.1.8. For a finite-dimensional $k$-vector space $V$, the group of invertible linear transformations $\mathrm{GL}(V)$ can be seen as the $k$-points of an algebraic $k$-group $\mathbf{G L}(V)$ defined as follows: For a fixed basis of $V, \mathbf{G L}(V)$ is the variety in $\mathbb{C}^{n^{2}+1}$ (with $n=\operatorname{dim} V$ ) defined by the vanishing of the polynomial $\operatorname{det}\left(x_{i j}\right) t-1 \in k\left[x_{11}, \ldots, x_{n n}, t\right]$, together with the following multiplication and inverse:

$$
(x, s)(y, t)=(x y, t s), \quad(x, t)^{-1}=(t C(x), \operatorname{det}(x))
$$

where $x y$ denotes matrix multiplication of $x$ and $y$, and $C(x)$ is the cofactor matrix of $x$. Then $\mathrm{GL}(V)=\mathbf{G L}(V)(k)$. Observe that the base extension $\mathbf{G L}(V)_{K}$ to the field $K$ is none else than $\mathbf{G L}(V \otimes K)$.

The group $\operatorname{SL}(V)$ of linear transformations of $V$ having determinant 1 also possesses a structure of linear algebraic group: it is the closed subgroup $\mathbf{S L}(V) \subset \mathbf{G L}(V)$ defined by:

$$
\mathbf{S L}(V)=\{(x, t) \in \mathbf{G L}(V) \mid t=1\} .
$$

We will usually consider elements of $\mathbf{G L}(V)$ as matrices and omit the last component $t$. In the special case where $V=k^{n}$, we will write $\mathbf{G L}_{n}$ instead of $\mathbf{G L}(V)$. 
Remark 2.1.9. A priori, the name affine algebraic group seems to be more justified in our definition of algebraic groups, the term linear being better suited for closed subgroups of $\mathbf{G L}_{n}$ for some $n$. It turns out that the two notions coincide, as the next theorem shows.

Theorem 2.3. Every linear (affine) algebraic group is isomorphic to a closed subgroup of some $\mathbf{G L}_{n}$.

Proof. Corollary 1.13 (ii) of [Br10].

We will be interested in specific algebraic groups called semisimple.

Definition 2.1.10. An algebraic group $\mathbf{G}$ is semisimple if $\mathbf{G}_{\mathbb{C}}$ has no nontrivial solvable connected normal subgroups. Furthermore, $\mathbf{G}$ is (absolutely) simple if $\mathbf{G}_{\mathbb{C}}$ has no nontrivial connected normal subgroups.

Remark 2.1.11. We follow [Co14] and [Vi71] and do not require semisimple groups to be connected. However, our definition reduces to the classical one in case $\mathbf{G}$ is connected. In particular, a group $\mathbf{G}$ is semisimple (in our definition) if and only if $\mathbf{G}^{\circ}$ is semisimple in the classical sense.

Next we define quotients of algebraic groups. To that end, we will consider a $k$-group $\mathbf{G}$ with a normal closed $k$-subgroup $\mathbf{N}$. We let $k[\mathbf{G}]^{\mathbf{N}}$ denote the following $k$-algebra:

$$
k[\mathbf{G}]^{\mathbf{N}}=\{f \in k[\mathbf{G}] \mid f(a x)=f(x) \text { for all } a \in \mathbf{N}, x \in \mathbf{G}\} .
$$

Theorem 2.4. Let $\mathbf{G}$ be an algebraic k-group, and $\mathbf{N} \subset \mathbf{G}$ a semisimple normal closed k-subgroup. Then:

1. The $k$-algebra $k[\mathbf{G}]^{\mathbf{N}}$ is finitely generated.

2. The group structure of $\mathbf{G}$ induces a linear algebraic group structure on the $k$-variety corresponding to $k[\mathbf{G}]^{\mathbf{N}}$; denote it by $\mathbf{G} / \mathbf{N}$.

3. The algebraic group $\mathbf{G} / \mathbf{N}$ is isomorphic (as groups) to the quotient of $\mathbf{G}$ by $\mathbf{N}$, and the quotient map $\mathbf{G} \rightarrow \mathbf{G} / \mathbf{N}$ defines a surjective closed morphism of algebraic groups.

Proof. This follows from [Br10, Theorem 1.24], which works in the more general case where $\mathbf{N}$ is reductive.

Definition 2.1.12. In the setting of Theorem 2.4, the quotient group $\mathbf{G} / \mathbf{N}$ with its structure of algebraic group is called the (categorical) quotient of $\mathbf{G}$ by $\mathbf{N}$.

We will actually only use quotients of algebraic groups in the special case where $\mathbf{N}$ is a finite central subgroup of $\mathbf{G}$, as in the next example. 
Example 2.1.13. Let $(V, f)$ be a quadratic space over $k$, that is, a $k$-vector space equipped with a quadratic form $f$. The orthogonal group $\mathrm{O}(V, f)$ of linear transformations of $V$ preserving $f$ can be naturally identified with the $k$-points of an algebraic group as follows. Define the orthogonal group $\mathbf{O}(V, f)$ (or simply $\mathbf{O}_{f}$ ) to be the following subgroup of $\mathbf{G L}(V)$ :

$$
\mathbf{O}_{f}=\mathbf{O}(V, f)=\{g=(g, t) \in \mathbf{G L}(V) \mid f \circ g=f\} .
$$

Here the defining condition $f \circ g=f$ is to be considered as a set of polynomial equations on the coefficients of $g$. Clearly we have a natural identification $\mathbf{O}_{f}(k)=\mathrm{O}(V, f)$. Similarly, one defines the special orthogonal group as

$$
\mathbf{S O}_{f}=\mathbf{S O}(V, f)=\{g \in \mathbf{O}(V, f) \mid \operatorname{det}(g)=1\},
$$

and finally, the projective orthogonal group as the quotient

$$
\mathbf{P O}_{f}=\mathbf{P O}(V, f)=\mathbf{O}_{f} /\{ \pm 1\} .
$$

Here \pm 1 denotes \pm the identity matrix in $\mathbf{O}_{f}$.

The groups $\mathbf{O}_{f}, \mathbf{S O}_{f}$ and $\mathbf{P O} \mathbf{O}_{f}$ are all semisimple $k$-groups if $\operatorname{dim}(V) \geq 3$, and (absolutely) simple if $\operatorname{dim}(V) \neq 4$.

Remark 2.1.14. If $k$ is algebraically closed, the group of $k$-points $\mathbf{P O}_{f}(k)$ of $\mathbf{P O}$ is simply $\mathbf{O}_{f}(k) / \pm 1$, that is, the image of $\mathbf{O}_{f}(k)$ in the quotient $\mathbf{P} \mathbf{O}_{f}$. However, this is no longer true (in general) if $k$ is not algebraically closed. This is because $\mathbf{P} \mathbf{O}_{f}(k)$ is (by definition) the image of the elements $g \in \mathbf{O}_{f}(\bar{k})$ such that $\sigma(g)= \pm g$ for all $\sigma \in \operatorname{Gal}(\bar{k} / k)$, which does not imply that $g$ is fixed by $\sigma$. The failure of the surjectivity of $\mathbf{O}_{f}(k) \rightarrow \mathbf{P O}_{f}(k)$ can be measured using Galois cohomology, see [Se68, p. 133].

Example 2.1.15. If $(V, f)$ is a symplectic space over $k$ (that is, a $k$-vector space equipped with an alternating form $f(x, y)$ ), the group $\operatorname{Sp}_{f}$ of linear transformations of $V$ preserving $f$ can also be seen as the $k$-points of an algebraic $k$-group $\mathbf{S p}_{f}$ by applying a similar construction as in the previous example. The group $\mathbf{S p}_{f}$ is then a simple $k$-group.

2.1.4 Dimension, tangent space, adjoint representation. In this section, we will briefly recall the notions of dimension, tangent spaces and smoothness of an algebraic variety. We will then use it to define the (algebraic) adjoint representation of an algebraic group. The reader is referred to [Ha77, Chapter 1, Section 5] for a precise treatment.

Definition 2.1.16. Let $V$ be a $k$-variety, with coordinate ring $k[V]$, and $x \in V$ be a point. The dimension $\operatorname{dim} V$ of $V$ is the maximal number $n$ such that there exists a strictly increasing tower of prime ideals

$$
0 \subsetneq \mathfrak{p}_{1} \subsetneq \cdots \subsetneq \mathfrak{p}_{n} \subsetneq k[V] .
$$


The maximal ideal $\mathfrak{m}_{x}$ of $x$ is the ideal in $k[V]$ of functions vanishing at $x$. The tangent space $T_{x} V$ of $V$ at $x$ is the $k$-vector space $\left(\mathfrak{m}_{x} / \mathfrak{m}_{x}^{2}\right)^{*}=\operatorname{Hom}\left(\mathfrak{m}_{x} / \mathfrak{m}_{x}^{2}, k\right)$. The variety $V$ is said to be smooth at the point $x$ if $\operatorname{dim}_{k} T_{x} V=\operatorname{dim} V$, and singular otherwise. Finally, $V$ is smooth if it is smooth at all points.

It turns out that the set of singular points can be characterized by polynomial equations, and that it does not cover the whole variety:

Theorem 2.5. The set of singular points of an algebraic variety is a proper closed subvariety.

Proof. See [Ha77, Th. 1.5.3].

Corollary 2.6. Any algebraic group is smooth.

Proof. Multiplication by an element $g$ of an algebraic group $\mathbf{G}$ is an isomorphism of algebraic varieties. Thus the tangent spaces at all points are isomorphic, and since $\mathbf{G}$ has a smooth point, all points are smooth.

Remark 2.1.17. If $V$ is a smooth $\mathbb{R}$-variety, then $V(\mathbb{R})($ resp. $V(\mathbb{C})$ ) possesses the structure of a (smooth) real (resp. complex) manifold (see [Mi13]). In that respect, the notions of dimension and tangent spaces are equivalent to those coming from the smooth manifold structure. In particular, if $\mathbf{G}$ is an algebraic $\mathbb{R}$-group, $\mathbf{G}(\mathbb{R})$ has the structure of a (smooth) real Lie group.

Definition 2.1.18. Let $\mathbf{G}$ be a $k$-group. The tangent space $T_{1} \mathbf{G}$ at the neutral element $1 \in \mathbf{G}$ is called the Lie algebra of $\mathbf{G}$, and usually denoted $\mathfrak{g}$.

Note that if $\mathbf{G}$ is a $k$-group, and $K / k$ is a field extension (with $K \subset \mathbb{C}$ ), then the Lie algebra of $\mathbf{G}_{K}$ is naturally isomorphic to $\mathfrak{g} \otimes_{k} K$.

Let $\mathbf{G}$ be a $k$-group, and $g \in \mathbf{G}(k)$. Then the map

$$
\begin{aligned}
\varphi_{g}: \mathbf{G} & \longrightarrow \mathbf{G} \\
x & \longmapsto g x g^{-1}
\end{aligned}
$$

is a $k$-isomorphism of algebraic groups. Therefore, it induces an isomorphism of rings $k[\mathbf{G}] \rightarrow k[\mathbf{G}]$ which sends the ideal $\mathfrak{m}_{1}$ to $\mathfrak{m}_{1}$ since $\varphi_{g}$ fixes $1 \in \mathbf{G}$. In turn, it induces an invertible linear transformation of $T_{1} \mathbf{G}=\mathfrak{g}$, which we denote by $\operatorname{Ad}(g)$. Since $g \in \mathbf{G}(k)$, we can see $\operatorname{Ad}(g)$ as an element of $\mathbf{G L}(\mathfrak{g})(k)$.

If $g \in \mathbf{G}(K)$ for a field extension $K / k$, the same process gives a linear transformation $\operatorname{Ad}(g)$ of $\mathfrak{g} \otimes_{k} K$, which defines, as before, an element of the group $\mathbf{G L}(\mathfrak{g} \otimes K)(K)=\mathbf{G L}(\mathfrak{g})(K)$. We can thus make the following definition.

Definition 2.1.19. Let $\mathbf{G}$ be a $k$-group. The adjoint representation of $\mathbf{G}$ is the linear representation

$$
\begin{aligned}
\mathrm{Ad}: \mathbf{G} & \longrightarrow \mathbf{G L}(\mathfrak{g}) \\
g & \longmapsto \operatorname{Ad}(g) .
\end{aligned}
$$


The map Ad is a morphism of algebraic groups which coincides with the adjoint representation on the real Lie group $\mathbf{G}(\mathbb{R})$ in case $\mathbf{G}$ is an algebraic $\mathbb{R}$-group.

Definition 2.1.20. An algebraic group $\mathbf{G}$ is adjoint if $\mathrm{Ad}$ is an isomorphism (of algebraic groups) onto its image.

Remark 2.1.21. This definition of adjoint groups is (as in the semisimple case) more general than the usual one, since our groups are not required to be connected. However, as before, one recovers the usual definition if the group is assumed to be connected, and if a group is adjoint in our sense then its identity component is adjoint in the classical sense.

Finally, we give a criterion for an algebraic group to be adjoint.

Proposition 2.7. An algebraic k-group $\mathbf{G}$ is adjoint if and only if the centralizer $\mathrm{Z}_{\mathbf{G}}\left(\mathbf{G}^{\circ}\right)$ of $\mathbf{G}^{\circ}$ in $\mathbf{G}$ is trivial.

Proof. Let $g \in \mathbf{G}(k)$. Then $g \in \mathrm{Z}_{\mathbf{G}}\left(\mathbf{G}^{\circ}\right)$ if and only if for every function $f \in k\left[\mathbf{G}^{\circ}\right]$, we have $\lambda_{g} f=f$, where $\lambda_{g} f$ is the function $x \mapsto f\left(g x g^{-1}\right)$. It is not hard to see that this holds exactly when $\lambda_{g} f=f$ for every linear (homogeneous degree 1) function $f \in \mathfrak{m}_{1}$, which is equivalent to $\overline{\lambda_{g} f}=\bar{f}$ for all $\bar{f} \in \mathfrak{m}_{1} / \mathfrak{m}_{1}^{2}$. In turn, this holds if and only if $\operatorname{Ad}(g)=1$.

2.1.5 Classical groups. The adjoint connected semisimple algebraic groups over $\mathbb{C}$ have been classified (see Tits [Ti66]). This classification extends with the help of Galois cohomology to the case of $k$-groups (for $k$ not necessarily algebraically closed). We will state (without proof) the result of this classification in the classical case, following Platonov and Rapinchuk [PR94].

Definition 2.1.22. Let $\mathbf{G}$ be an algebraic group over $\mathbb{C}$, and let $k \subset \mathbb{C}$ be a subfield. Then a $k$-form of $\mathbf{G}$ is an algebraic $k$-group $\mathbf{F}$ such that $\mathbf{F}_{\mathbb{C}}=\mathbf{G}$. If $\mathbf{G}$ is connected, adjoint and simple, then the type of $\mathbf{G}$ is the isomorphism type of its root system, as defined in [Ti66]. If $\mathbf{G}$ is an absolutely simple $k$-group, we extend this definition by saying that the type of $\mathbf{G}$ is the type of $\operatorname{Ad}\left(\mathbf{G}_{\mathbb{C}}\right)^{\circ}$.

The irreducible root systems are classified by their Dynkin diagrams, and fall into one of the infinite families $A_{n}, B_{n}, C_{n}, D_{n}$ or into one of the exceptional systems $E_{6}, E_{7}, E_{8}, F_{4}$ and $G_{2}$ :

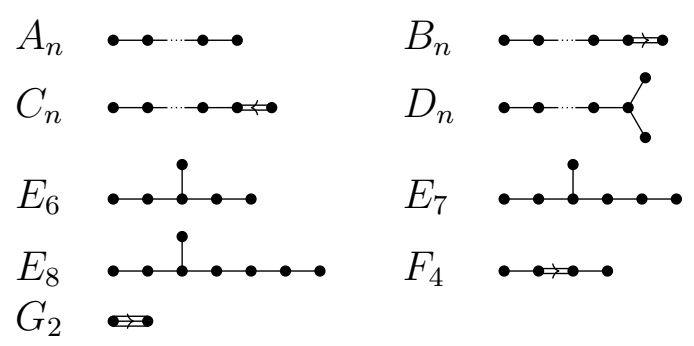


Definition 2.1.23. A group $\mathbf{G}$ is classical if its root system is $A_{n}, B_{n}, C_{n}$ or $D_{n}$.

We will give the list of (almost) all classical connected absolutely simple adjoint $k$-groups in case $k$ is an number field. First, we will need two more examples of algebraic groups.

Example 2.1.24. Let $D$ be a central division algebra over the number field $k$, of degree $d$ (i.e., the center of $D$ is $k$, and the index $[D: k]$ is $d^{2}$ ). The set $\mathrm{SL}_{n}(D)$ of $n \times n$ matrices with coefficients in $D$ and reduced norm Nrd 1 forms a group (under standard matrix multiplication). We will realize it as the $k$-points of an algebraic $k$-group.

Consider $D$ seen as a $k$-vector space and fix a $k$-basis. Then to each element $a \in D$ corresponds a matrix $\widehat{a} \in \operatorname{Mat}_{d^{2}}(k)$, given by the matrix of the linear map $D \rightarrow D ; x \mapsto a x$. The image $\widehat{D}$ of $D$ in $\operatorname{Mat}_{d^{2}}(k)$ is a linear subspace of $\operatorname{Mat}_{d^{2}}(k) \cong k^{d^{4}}$, and is thus determined by linear equations with coefficients in $k$. Identifying $\operatorname{Mat}_{d^{2}}(\mathbb{C})$ with $\mathbb{C}^{d^{4}}$ and seeing $\operatorname{Mat}_{d^{2}}(k) \subset \operatorname{Mat}_{d^{2}}(\mathbb{C})$, these equations endow $\widehat{D}$ with a structure of affine $k$-variety, with a canonical identification $\widehat{D}(k)=D$.

In turn, we have a natural identification of $A=\operatorname{Mat}_{n}(D)$ with

$$
\widehat{A}(k)=\operatorname{Mat}_{n}(\widehat{D}(k)) \subset \operatorname{Mat}_{n d^{2}}(k) .
$$

Again, this shows that $\widehat{A}$ has a structure of $k$-variety, with $\widehat{A}(k)=A$. Finally, one can show that the reduced norm Nrd corresponds to a polynomial map $\widehat{\operatorname{Nrd}}: \widehat{A}(k) \rightarrow k$. Thus we can identify $\operatorname{SL}_{n}(D)=\{x \in A \mid \operatorname{Nrd}(x)=1\}$ with the $k$-points of

$$
\mathbf{S L}_{n}(D)=\{x \in \widehat{A} \mid \widehat{\operatorname{Nrd}}(x)=1\} \text {. }
$$

With this definition, $\mathbf{S L}_{n}(D)$ is a semisimple algebraic $k$-group.

Example 2.1.25. Let $\ell$ be a number field, and let $D$ be a central division algebra over $\ell$ Assume that it has an involution $\tau$ (that is, an anti-automorphism of $D$ such that $\left.\tau^{2}=\mathrm{id}\right)$. Let $k=\ell^{\tau}$ be the fixed field of $\tau$, let $d$ denote the degree of $D$ (so that $[D: \ell]=d^{2}$ ) and let $l=[\ell: k]$. Finally, let $h=h(x, y)$ be a non-degenerate Hermitian or skew-Hermitian sesquilinear form on $V=D^{n}$. The unitary group $\mathrm{U}(D, h)$ is the group of invertible linear transformations of $V$ which preserve $h$, and the special unitary group $\mathrm{SU}(D, h)$ is the subgroup of $\mathrm{U}(D, h)$ of elements having reduced norm 1 . If a basis $e_{1}, \ldots, e_{n}$ of $V$ has been chosen, the group $\mathrm{U}(D, h)$ can be written as

$$
\mathrm{U}(D, h)=\left\{\left.g \in \mathrm{GL}_{n}(D)\right|^{*} g H g=H\right\} .
$$

where $H=\left(h\left(e_{i}, e_{j}\right)_{i j}\right)$ and ${ }^{*} g=\left(\tau\left(g_{j i}\right)\right)$ if $g=\left(g_{i j}\right)$. 
In a similar fashion as in Example 2.1.24, one can realize $\mathrm{U}(D, h)$ and $\mathrm{SU}(D, h)$ as the $k$-points of algebraic $k$-groups $\mathbf{U}(D, h)$ and $\mathbf{S U}(D, h)$ respectively. As before, we can take the quotient of the groups by $\{ \pm 1\}$ to obtain

$$
\mathbf{P U}(D, h)=\mathbf{U}(D, h) /\{ \pm 1\} .
$$

These groups are all semisimple.

We end this section by giving the list of all classical adjoint connected semisimple $k$-groups of type different from $D_{4}$ (see the end of Section 2.3.4 of [PR94]).

Theorem 2.8. Let $\mathbf{G}$ be an absolutely simple connected algebraic $k$-group, for $k$ a number field. Then unless $\mathbf{G}$ is of type $D_{4}$, the adjoint form $\mathrm{Ad} \mathbf{G}$ of $\mathbf{G}$ is isomorphic to the adjoint form of a group in the following list:

1. Type $A_{n}(n \geq 1)$ :

(a) $\mathbf{S L}_{m}(D)$ for a division algebra $D$ over $k$ of index $d$ and $n=m d-1$.

(b) $\mathbf{S U}(D, h)$ where $D$ is a division algebra of index $d$ over a field $\ell$ with an involution $\tau$ such that $k=\ell^{\tau} \subsetneq \ell, h$ a hermitian form of dimension $m$ and $n=m d-1$.

2. Type $B_{n}(n \geq 1)$ :

(a) $\mathbf{S O}_{f}$ where $f$ is a quadratic form of dimension $2 n+1$.

3. Type $C_{n}(n \geq 1)$ :

(a) $\mathbf{S p}_{f}$ where $f$ is an alternating form of dimension $2 n$.

(b) $\mathbf{S U}(D, h)$ where $D$ is a quaternion algebra over $k$ with canonical involution $\tau$ fixing $k$ and $h$ a Hermitian form of dimension $n$.

4. Type $D_{n}(n \geq 3)$ :

(a) $\mathbf{S O}_{f}$ where $f$ is a quadratic form of dimension $2 n$.

(b) $\mathbf{S U}(D, h)$ where $D$ is a quaternion algebra over $k$ with canonical involution $\tau$ fixing $k$ and $h$ a skew-Hermitian form of dimension $n$.

Remark 2.1.26. The Dynkin diagram $D_{4}$ has an additional automorphism of order 3 , which is not present in $D_{n}$ for $n \neq 3$. This implies the existence of a special kind of algebraic $k$-groups coming from so-called trialitarian algebras which are not listed above. As these will be of no interest to us, we will not define them; we refer to [INV98, §44] for a detailed account on those groups. 
2.1.6 Automorphisms of $\mathbf{P O} \mathbf{O}_{f}$. The group $\mathbf{P} \mathbf{O}_{f}$ defined in Example 2.1.13 will play a central role in this thesis. We investigate some of its properties in this section. Let $f$ be a quadratic form in $n$ variables over a field $k$. Observe first that the group $\mathbf{P O}_{f}$ is:

1. connected if $n$ is odd. This follows from the fact that $\mathbf{S O}_{f}$ is connected and $-1 \notin \mathbf{S O}_{f}$, so we have

$$
\mathbf{P O}_{f} \cong \mathrm{SO}_{f}
$$

2. disconnected if $n$ is even. In that case, the determinant det: $\mathbf{O}_{f} \rightarrow \mathbb{C}$ is a polynomial map defined over $k$, and it induces a polynomial map on $\mathbf{P O}_{f}$ which takes values 1 or -1 . The connected component $\mathbf{P} \mathbf{O}_{f}^{\circ}$ of $\mathbf{P O}_{f}$ is thus the quotient of $\mathbf{S O}_{f}$ by $\{ \pm 1\}$, a group sometimes denoted by $\mathbf{P S O}_{f}$.

We start our investigation of these groups and their automorphisms with a basic lemma.

Lemma 2.9. Let $f$ be a quadratic form in $n \geq 3$ variables and let $g \in \mathbf{G L}_{n}$ be such that

$$
g u= \pm u g \quad \text { for all } u \in \mathbf{S O}_{f} .
$$

Then $g=\lambda I$, for some $\lambda \in \mathbb{C}^{\times}$.

Proof. Since we are working over $\mathbb{C}$, we can assume that $f$ is the standard positive definite quadratic form, so that $\mathbf{S O}_{f}=\mathbf{S O}_{n}$. Using equation (2.1) with conjugates of $u=\operatorname{diag}(-1,-1,1, \ldots, 1) \in \mathbf{S O}_{n}$ gives that $g$ is diagonal (except if $n=4$, where matrices of the form

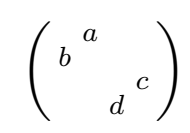

can also arise). Using (2.1) again with (even) permutation matrices gives that all coefficients on the diagonal must be equal (and excludes the above special matrices in the case $n=4$ ).

Since the connected component $\mathbf{P} \mathbf{O}_{f}^{\circ}$ of $\mathbf{P} \mathbf{O}_{f}$ is the image of $\mathbf{S O}_{f}$ in the quotient $\mathbf{P O}_{f}$, an application of Proposition 2.7 directly gives:

Corollary 2.10. If $f$ has $n \geq 3$ variables, then $\mathbf{P O}_{f}$ is adjoint.

We continue by determining the (algebraic) automorphisms of $\mathbf{P O}_{f}$.

Theorem 2.11. Let $f$ be a quadratic form in $n \geq 4$ variables over a number field $k$. Then any (algebraic) $k$-automorphism of $\mathbf{P} \mathbf{O}_{f}$ is inner, that is, given by

$$
x \mapsto g x g^{-1} \quad \text { for some } g \in \mathbf{P O}_{f}(k) .
$$


Proof. Let $\varphi: \mathbf{P O}_{f} \rightarrow \mathbf{P O}_{f}$ be a $k$-automorphism, and let $\ell / k$ be a quadratic extension such that $f$ becomes isotropic over $\ell$ (i.e. there exists $x \in \ell^{n}$ with $f(x)=0$ ). By the classification of automorphisms of $\mathbf{P O}_{f}(\ell)$ (see Dieudonné [Di80, XII]), $\varphi$ is induced by an automorphism of $\mathbf{O}_{f}(\ell)$ of the form

$$
x \mapsto \chi(x) h x h^{-1},
$$

where $\chi: \mathbf{O}_{f}(\ell) \rightarrow\{ \pm 1\}$ is a group homomorphism and $h$ is a semi-linear invertible transformation (with respect to an automorphism $\sigma$ of $\ell$ ) such that $f(h x)=\lambda \sigma(f(x))$. Since we are working with $\mathbf{P O}_{f}$ we can omit $\chi$, and since $\varphi$ is algebraic, we see that $h$ must be linear. It follows that $f \circ h=\lambda f$, and thus

$$
\widetilde{g}=\frac{1}{\sqrt{\lambda}} h \in \mathbf{O}_{f}(\ell(\sqrt{\lambda}))
$$

is such that $\varphi(x)=\widetilde{g} x \widetilde{g}^{-1}$. Since $\sigma(\widetilde{g})= \pm \widetilde{g}$ for any $\sigma \in \operatorname{Gal}(\ell(\sqrt{\lambda}) / \ell)$, its image $g$ is in $\mathbf{P O}_{f}(\ell)$. Finally, if $\tau \in \operatorname{Gal}(\ell / k)$ is the non-trivial automorphism, we have ${ }^{\tau} \varphi=\varphi$ since $\varphi$ is defined over $k$. As ${ }^{\tau} \varphi(x)$ is conjugation by ${ }^{\tau} g$, it follows that ${ }^{\tau} g g^{-1}$ commutes with every element of $\mathbf{P O}$. Since $\mathbf{P} \mathbf{O}_{f}$ is adjoint, we have ${ }^{\tau} g=g$, and thus $g \in \mathbf{P O}_{f}(k)$.

Remark 2.1.27. If $f$ has $n=2 m+1$ variables, the group $\mathbf{P O} \mathbf{O}_{f}$ is connected and of type $B_{m}$, and therefore all automorphisms are inner (since the Dynkin diagram has no non-trivial automorphisms). If however $f$ has $n=2 m$ variables, the group $\mathbf{P} \mathbf{O}_{f}$ has two connected components, and $\mathbf{P} \mathbf{O}_{f}^{\circ}$ is of type $D_{m}$. Hence there exists (at least) one outer automorphism of $\mathbf{P} \mathbf{O}_{f}^{\circ}$ induced by a non-trivial automorphism of the Dynkin diagram. However, this is consistent with Theorem 2.11, since in that case the outer automorphism of $\mathbf{P O}_{f}^{\circ}$ is induced via conjugation by an element of $\mathbf{P O}_{f} \backslash \mathbf{P O}_{f}^{\circ}$.

We will now investigate various corollaries of Theorem 2.11 .

Corollary 2.12. Any automorphism $\varphi: \mathbf{P O}_{f, \mathbb{C}} \rightarrow \mathbf{P O}_{f, \mathbb{C}}$ with $\left.\varphi\right|_{\mathbf{P O}} \mathbf{O}_{f, \mathbb{C}}=\mathrm{id}$ is trivial, that is, $\varphi=\mathrm{id}$.

Proof. Any such automorphism is of the form $x \mapsto g x g^{-1}$ for $g \in \mathbf{P O}_{f}$, by Theorem 2.11. By hypothesis, $g$ is in the centralizer $\mathbf{Z}_{\mathbf{P O}_{f}}\left(\mathbf{P O}_{f}^{\circ}\right)$ of $\mathbf{P} \mathbf{O}_{f}^{\circ}$ in $\mathbf{P} \mathbf{O}_{f}$, and thus $g=1$ since $\mathbf{P} \mathbf{O}_{f}$ is adjoint.

Using this corollary, one can extend the result of Corollary 2.2 to the case of almost Zariski-dense subsets $\mathbf{P O}_{f}(k)$, i.e., subsets $\Gamma$ such that $\Gamma \cap \mathbf{P O}_{f}^{\circ}$ is Zariski-dense in $\mathbf{P O}_{f}^{\circ}$.

Proposition 2.13. Let $f$ be a quadratic form over $k$ in $n \geq 4$ variables, and let $\Gamma \subset \mathbf{P O}_{f}^{\circ}(k)$ be Zariski-dense (in $\mathbf{P O}_{f}^{\circ}$ ). Let $\mathbf{G}$ be an algebraic k-group and $\varphi: \mathbf{P O}_{f, \mathbb{C}} \rightarrow \mathbf{G}_{\mathbb{C}}$ be a $\mathbb{C}$-isomorphism such that $\varphi(\Gamma) \subset \mathbf{G}(k)$. Then $\varphi$ is defined over $k$. 
Proof. By Corollary 2.2, $\left.\varphi\right|_{\mathbf{P O}_{f, \mathbb{C}}^{\circ}}: \mathbf{P O}_{f, \mathbb{C}}^{\circ} \rightarrow \mathbf{G}_{\mathbb{C}}^{\circ}$ is an isomorphism defined over $k$. Thus for any automorphism $\sigma$ of $\mathbb{C}$ fixing $k$, we have $\left.{ }^{\sigma} \varphi^{-1} \circ \varphi\right|_{\mathbf{P O}_{f, \mathbb{C}}^{\circ}}=$ id. By Corollary 2.12 this holds as well over $\mathbf{P O}_{f, \mathbb{C}}$ and we have ${ }^{\sigma} \varphi=\varphi$. Since $\sigma$ was arbitrary, $\varphi$ is defined over $k$ by Lemma 2.1 .

Finally, a last result about the shape of isomorphisms $\mathbf{P O} \mathbf{f}_{1} \cong \mathbf{P O}_{f_{2}}$.

Theorem 2.14. Let $f_{1}, f_{2}$ be two quadratic forms with $n \geq 4$ variables defined over a number field $k$. Then any $k$-isomorphism $\mathbf{P O}_{f_{1}} \cong \mathbf{P} \mathbf{O}_{f_{2}}$ is induced by a $k$-similitude $f_{1} \cong \lambda f_{2}$ for some $\lambda \in k$. In particular, the groups are isomorphic if and only if the forms are similar.

Proof. If $f_{1} \circ A=\lambda f_{2}$, for some $A \in \mathbf{G L}_{n}(k)$, then conjugation by $A$ gives the required isomorphism of the groups. For the other part, let $\varphi: \mathbf{P O}_{f_{1}} \rightarrow \mathbf{P O}_{f_{2}}$ be an isomorphism, and let $A \in \mathbf{G L}_{n}(K)$ be a matrix such that $f_{1} \circ A=f_{2}$, for some Galois extension $K / k$. By Theorem 2.11, there exists (up to increasing $K$ ) some $g \in \mathbf{O}_{f}(K)$ such that the composition of $\varphi$ with conjugation by $A$ is conjugation by $g$. Therefore, $\varphi$ is itself induced via conjugation by some matrix $B \in \mathbf{G L}_{n}(K)$.

Since $\varphi$ is defined over $k$, the image of $B$ is in $\mathbf{P G} \mathbf{L}_{n}(k)$. From the exact sequence

$$
1 \rightarrow \mathbf{G}_{m} \rightarrow \mathbf{G L}_{n} \rightarrow \mathbf{P G L}_{n} \rightarrow 1
$$

and the fact that $H^{1}\left(k, \mathbf{G}_{m}\right)=1$ (Hilbert's theorem 90, see [Se68, Proposition 2, p. 158]), we obtain that the map $\mathbf{G L}_{n}(k) \rightarrow \mathbf{P} \mathbf{G L}_{n}(k)$ is surjective (see [Se68, Proposition 1, p. 133]). Thus some multiple $C$ of $B$ is in $\mathbf{G L}_{n}(k)$, and the matrix $C$ induces the required similitude.

\section{$2.2 \mid$ Hyperbolic geometry}

We will now give the basic elements of hyperbolic geometry needed in the thesis. We will assume basic knowledge of Riemannian geometry. In particular, the notions of Riemannian metric, geodesic, curvature will not be introduced. We refer to Benedetti-Petronio [BP89], Ratcliffe [Ra06] as well as Martelli [Ma16] for a detailed introduction.

2.2.1 Hyperbolic manifolds. We start by defining hyperbolic manifolds.

Definition 2.2.1. A hyperbolic manifold is a complete Riemannian manifold (without boundary) of constant sectional curvature -1 .

Up to isometry, the hyperbolic space $\mathbf{H}^{n}$ is the unique simply connected hyperbolic manifold. It can be constructed using the hyperboloid model as follows. Consider the standard quadratic form $f_{n, 1}=-x_{0}^{2}+x_{1}^{2}+\cdots+x_{n}^{2}$ of 
signature $(n, 1)$ and let $\langle\cdot, \cdot\rangle_{n, 1}$ denote the induced bilinear form on $\mathbb{R}^{n+1}$. The level set $H=\left\{x \in \mathbb{R}^{n+1} \mid f_{n, 1}(x)=-1\right\}$ consists of a two sheeted hyperboloid. By identifying the tangent space at $x \in H$ with the orthogonal complement

$$
x^{\perp_{n, 1}}=\left\{y \in \mathbb{R}^{n+1} \mid\langle x, y\rangle_{n, 1}=0\right\},
$$

we see that the form $\langle\cdot, \cdot\rangle_{n, 1}$ induces a positive definite bilinear form on this tangent space which varies smoothly with $x$. Thus we obtain a Riemannian metric on it, and one can verify by computing the curvature tensor that all sectional curvatures are equal to -1 .

In order to make it a connected space, we have two options. Either we simply take one of the two sheets of $H$ (by taking only elements with positive $x_{0}$ coordinate, for example), or we identify opposite points on the two sheets together, that is, we quotient by the action of the group $\{ \pm 1\}$. The latter method permits a natural identification of the isometry group as an algebraic group, as we will see shortly; it is therefore the convention that we will adopt in this thesis. To sum up, we have:

$$
\mathbf{H}^{n} \cong H /\{ \pm 1\} \text {, with the Riemannian metric induced from }\langle\cdot, \cdot\rangle .
$$

Using this model, the group of isometries of $\mathbf{H}^{n}$ is:

$$
\operatorname{Isom}\left(\mathbf{H}^{n}\right) \cong \mathrm{PO}(n, 1)=\mathrm{O}(n, 1) /\{ \pm 1\},
$$

where

$$
\mathrm{O}(n, 1)=\left\{g \in \mathrm{GL}_{n+1}(\mathbb{R}) \mid\langle g x, g y\rangle_{n, 1}=\langle x, y\rangle_{n, 1} \text { for all } x, y\right\} .
$$

Observe that the Lie group $\mathrm{PO}(n, 1)$ canonically admits a structure of real algebraic group defined as

$$
\mathbf{P O}_{(n, 1)}=\mathbf{P O}_{f_{n, 1}, \mathbb{R}}, \quad \text { where } f_{n, 1}=-x_{0}^{2}+x_{1}^{2}+\cdots+x_{n}^{2} .
$$

For $n \geq 2$, we have a natural identification $\operatorname{PO}(n, 1)=\mathbf{P O}_{(n, 1)}(\mathbb{R})$. Indeed, the group $\mathbf{P O}_{(n, 1)}(\mathbb{R})$ is (by definition) the image of the elements $g \in \mathbf{O}_{f_{n, 1}}(\mathbb{C})$ such that $\sigma(g)= \pm g$, where $\sigma$ denotes complex conjugation. If $\sigma(g)=-g$, we see that $g=i h$, where $h$ is a real matrix which must send $f_{(n, 1)}$ to $-f_{(n, 1)}$. Since the latter has signature $(1, n)$, this is impossible, and thus $\sigma(g)=g$. It follows that $\mathbf{P O}_{(n, 1)}(\mathbb{R})=\mathbf{O}_{f_{n, 1}}(\mathbb{R}) / \pm 1=\mathrm{PO}(n, 1)$. This will be especially useful in Section 3.1.

Since $\mathbf{H}^{n}$ is the unique simply connected hyperbolic manifold, it follows from the theory of covering spaces that every (complete) hyperbolic manifold has $\mathbf{H}^{n}$ as a universal cover. More precisely, for each hyperbolic manifold $M$, there exists a covering map $\mathbf{H}^{n} \rightarrow M$ such that for every other covering $M^{\prime} \rightarrow M$ by a connected hyperbolic manifold $M^{\prime}$, the covering of $M$ factors as $\mathbf{H}^{n} \rightarrow M^{\prime} \rightarrow M$, where $\mathbf{H}^{n} \rightarrow M^{\prime}$ is the covering map corresponding to $M^{\prime}$. 
It follows that the fundamental group $\pi_{1}(M)$ of $M$ is isomorphic to the group $\Gamma$ of deck transformations of the covering $\mathbf{H}^{n} \rightarrow M$. This group $\Gamma$ is a discrete torsion-free subgroup of $\mathrm{PO}(n, 1)$ and the quotient $\Gamma \backslash \mathbf{H}^{n}$ has a natural structure of hyperbolic manifold such that $M \cong \Gamma \backslash \mathbf{H}^{n}$. This works for general discrete torsion-free subgroups of $\mathrm{PO}(n, 1)$, whence the following:

Theorem 2.15. There is a correspondence between complete hyperbolic manifolds $M$ and discrete torsion-free subgroups $\Gamma \subset \mathrm{PO}(n, 1)$. It associates a manifold $M$ with the group of deck transformations $\Gamma$ of a covering $\mathbf{H}^{n} \rightarrow M$, and a discrete torsion-free subgroup $\Gamma \subset \mathrm{PO}(n, 1)$ with the manifold $M=\Gamma \backslash \mathbf{H}^{n}$. Furthermore, it takes isometric manifolds to conjugate subgroups, and a manifold $M$ has finite volume if and only if $\Gamma$ has finite covolume, i.e., is a lattice in $\mathrm{PO}(n, 1)$.

If one is interested in the study of hyperbolic manifolds, this theorem reduces the problem to the study of torsion-free lattices in $\operatorname{PO}(n, 1)$. In fact, in dimension $n \geq 3$ it is enough to know the isomorphism type of a subgroup $\Gamma \subset \mathrm{PO}(n, 1)$, as the next theorem shows.

Theorem 2.16 (Mostow Rigidity Theorem). Let $M, M^{\prime}$ be two hyperbolic $n$-manifolds, for $n \geq 3$. Assume there is an isomorphism $\alpha: \pi_{1}(M) \rightarrow \pi_{1}\left(M^{\prime}\right)$. Then $\alpha$ is actually induced by an isometry $\phi: M \rightarrow M^{\prime}$.

We end this section by a proposition on isometries of hyperbolic manifolds.

Proposition 2.17. Let $M=\Gamma \backslash \mathbf{H}^{n}$ be a hyperbolic manifold. Then there is an isomorphism

$$
N(\Gamma) / \Gamma \stackrel{\sim}{\longrightarrow} \operatorname{Isom}(M),
$$

where $N(\Gamma)$ denotes the normalizer of $\Gamma$ in $\mathrm{PO}(n, 1)$.

Proof. By properties of the universal coverings, an isometry $\varphi: M \rightarrow M$ lifts to an isometry $g: \mathbf{H}^{n} \rightarrow \mathbf{H}^{n}$, i.e., an element $g \in \mathrm{PO}(n, 1)$, which is unique up to multiplying with an element of $\Gamma$. Since $M=\Gamma \backslash \mathbf{H}^{n}$, we have $g(\Gamma x)=\Gamma g x$ for each $x \in \mathbf{H}^{n}$. Thus $g \Gamma=\Gamma g$, i.e., $g$ normalizes $\Gamma$. On the other hand, any isometry $g \in \mathrm{PO}(n, 1)$ normalizing $\Gamma$ induces an isometry of $M$. Finally, the trivial isometry clearly corresponds to elements of $\Gamma$.

2.2.2 Hyperplanes and boundary. In order to study gluing constructions we will need to speak of hyperbolic manifolds with totally geodesic boundary, which we now introduce.

Definition 2.2.2. A submanifold $M \subset M^{\prime}$ of a complete Riemannian manifold $M^{\prime}$ is called totally geodesic if every maximal geodesic in $M$ (with the Riemannian metric induced from $M^{\prime}$ ) is also a maximal geodesic in $M^{\prime}$. A hyperbolic subspace of $\mathbf{H}^{n}$ is a connected totally geodesic submanifold of $\mathbf{H}^{n}$. A hyperplane is a proper hyperbolic subspace of maximal dimension. 
It is not hard to show that any hyperplane in $\mathbf{H}^{n}$ is the image $\bmod \{ \pm 1\}$ of the level set $H$ intersected with a codimension one linear subspace of $\mathbb{R}^{n+1}$. More precisely, each hyperplane $R \subset \mathbf{H}^{n}$ is obtained as

$$
R=\left(x^{\perp_{n, 1}} \cap H\right) /\{ \pm 1\} \quad \text { for some } x \in \mathbb{R}^{n+1} \text { with } f_{n, 1}(x)>0 .
$$

Thus a hyperplane separates $\mathbf{H}^{n}$ into two components, and we can make the following definition:

Definition 2.2.3. A half-space in $\mathbf{H}^{n}$ is the closure of one of the two connected components of $\mathbf{H}^{n} \backslash R$ for a hyperplane $R$.

Equivalently, a half-space can be obtained as

$$
L^{ \pm}=\{x \in H \mid \pm\langle x, v\rangle \geq 0\} /\{ \pm 1\} \quad \text { for some } x \in \mathbb{R}^{n+1} \text { with } f_{n, 1}(x)>0 .
$$

Observe that all half-spaces are isometric to each other, and all hyperplanes are isometric to $\mathbf{H}^{n-1}$ (one can see this by completing $x$ to an orthogonal basis of the quadratic space $\left(\mathbb{R}^{n+1}, f\right)$ ). We are ready to define hyperbolic manifolds with boundary.

Definition 2.2.4. A hyperbolic manifold with (totally geodesic) boundary is a Riemannian manifold with boundary that is locally isometric to a half-space $L \subset \mathbf{H}^{n}$, which is complete and with totally geodesic boundary components.

The main example of hyperbolic manifold with boundary we will use in this thesis is the following:

Definition 2.2.5. Let $M$ be a hyperbolic manifold. A hypersurface of $M$ is a (connected) submanifold $N \subset M$ of codimension 1 which is complete without boundary and totally geodesic. A (hyperbolic) piece of $M$ is the completion of a connected component of $M \backslash \bigsqcup_{i=1}^{r} N_{i}$, where $N_{1}, \ldots, N_{r}$ are disjoint hypersurfaces of $M$.

Remark 2.2.6. By definition, hypersurface means embedded hypersurface. It will however be useful to consider also immersed hypersurfaces, by which we mean the image under an immersion of a codimension 1 complete manifold without boundary.

It is not hard to see that a hyperbolic piece is a hyperbolic manifold with boundary. In fact, one can even describe its universal cover as follows.

Proposition 2.18. Let $M$ be a hyperbolic manifold and let $\pi: \mathbf{H}^{n} \rightarrow M$ be a universal covering with group of deck transformations $\Gamma \subset \mathrm{PO}(n, 1)$, so that $M \cong \Gamma \backslash \mathbf{H}^{n}$. 
1. Let $N \subset M$ be a hypersurface. Then the pre-image $\pi^{-1}(N) \subset \mathbf{H}^{n}$ is a disjoint union of hyperplanes of $\mathbf{H}^{n}$. If $R$ is such a hyperplane, then $\pi^{-1}(N)=\bigcup_{\gamma \in \Gamma} \gamma R$. Moreover, the restriction $\left.\pi\right|_{R}: R \rightarrow N$ is a universal covering which induces an isometry

$$
N \cong \operatorname{Stab}_{\Gamma}(R) \backslash R
$$

2. Let $N_{1}, \ldots, N_{r}$ be disjoint hypersurfaces in $M$, let $C$ be a connected component of $M \backslash \bigsqcup N_{i}$ and let $M_{0}$ be the completion of $C$. The closure $\overline{\pi^{-1}(C)} \subset \mathbf{H}^{n}$ of the pre-image of $C$ is an interior-disjoint union of intersection of half-spaces whose boundary hyperplanes are universal coverings of the $N_{i}$. If $\widetilde{M}_{0}$ is such an intersection of half-spaces, then $\overline{\pi^{-1}(C)}=\bigcup_{\gamma \in \Gamma} \gamma \widetilde{M}_{0}$. Moreover, the restriction of $\pi$ to the interior of $\widetilde{M}_{0}$ induces a universal covering $\left.\pi\right|_{\widetilde{M}_{0}}: \widetilde{M}_{0} \rightarrow M_{0}$ on the completions, and we have

$$
M_{0} \cong \operatorname{Stab}_{\Gamma}\left(\widetilde{M}_{0}\right) \backslash \widetilde{M}_{0}
$$

Proof. We sketch a proof for the sake of completeness (see [Ma16, §3.5.1] for a more precise one). Since $N$ is totally geodesic, so is any connected covering, thus connected components of $\pi^{-1}(N)$ must be hyperbolic subspaces, hence hyperplanes for dimension reasons. If $R$ is such a hyperplane, all its $\Gamma$ translates must either be disjoint or coincide with $R$, as $N$ is embedded in $M$. Furthermore, since $\Gamma$ is the group of deck transformations of the covering $\pi$, it follows that such $\Gamma$ translates must exhaust $\pi^{-1}(N)$. Because any hyperplane is simply connected, the restriction of $\pi$ to such a hyperplane $R$ is a universal covering. Its group of deck transformations is easily seen to be $\operatorname{Stab}_{\Gamma}(R)$, and thus part 1 follows.

For part 2, the proof is almost the same. A connected component of $\pi^{-1}(C)$ corresponds to a connected component of $\pi^{-1}\left(M \backslash \bigsqcup N_{i}\right)=\mathbf{H}^{n} \backslash \bigcup_{i, \gamma} \gamma R_{i}$, where $R_{i}$ is a hyperplane in $\pi^{-1}\left(N_{i}\right)$, which is an intersection of half-spaces of the required form. The rest of the arguments are the same as in part 1.

The following construction implies that a hyperbolic manifold with boundary is always a piece of a manifold.

Definition 2.2.7. Let $M_{0}$ be a manifold with totally geodesic boundary. The double of $M_{0}$ is the manifold $M_{0} \times\{0,1\} / \sim$ where $(x, 0) \sim(x, 1)$ for $x \in \partial M_{0}$.

The double of a hyperbolic manifold with totally geodesic boundary is indeed a hyperbolic manifold, by [Ma16, Prop. 3.5.4] (alternatively, this will follow from Theorem 4.1 below).

Remark 2.2.8. By construction, a hypersurface locally separates a manifold into two parts. Thus if $M_{0}$ is a piece of $M$, say the completion of $M \backslash \bigsqcup N_{i}$, the natural map $M_{0} \rightarrow M$ induces a double-cover of each $N_{i}$. If the normal 
bundle of $N_{i} \subset M$ is orientable, this translates as there being two copies of $N_{i}$ in $\partial M_{0}$, i.e., the double-cover is trivial (it consists of two copies of $N_{i}$ ). However, if the normal bundle of $N_{i} \subset M$ is non-orientable, then the doublecover is not trivial, and $N_{i} \subset M$ lifts to one connected component $\widetilde{N}_{i} \subset \partial M_{0}$. This happens exactly when $M$ is orientable and $N_{i}$ is not or, vice-versa, when $M$ is non-orientable and $N_{i}$ is.

2.2.3 Commensurability and torsion. If the lattice $\Gamma \subset \mathrm{PO}(n, 1)$ has torsion, it will have fixed points in $\mathbf{H}^{n}$, and thus the quotient $\Gamma \backslash \mathbf{H}^{n}$ will not be a manifold (but a so-called hyperbolic orbifold). However, one can still obtain a finite-volume manifold from this lattice using the following theorem:

Theorem 2.19 (Selberg's Lemma). Any subgroup $\Gamma \subset \mathrm{PO}(n, 1)$ that is finitely generated has a torsion-free subgroup of finite index.

This theorem is actually valid for any finitely generated subgroup of $\mathrm{GL}_{n}(\mathbb{C})$. Since all lattices in $\mathrm{PO}(n, 1)$ are finitely generated (a highly non-trivial fact, see [Ra72, Remark 6.18 and Theorem 13.15]), we see that a lattice in $\operatorname{PO}(n, 1)$ determines a commensurability class of hyperbolic manifolds.

Definition 2.2.9. Two hyperbolic manifolds $M_{1}, M_{2}$ are commensurable if they admit a common finite cover $M$.

The dual notion for lattices is the following.

Definition 2.2.10. Two subgroups $\Gamma_{1}, \Gamma_{2} \subset \mathrm{PO}(n, 1)$ are strictly commensurable if $\Gamma_{1} \cap \Gamma_{2}$ is of finite index both in $\Gamma_{1}$ and $\Gamma_{2}$. They are commensurable (or commensurable in the wide sense) if $\Gamma_{1}$ is strictly commensurable to a conjugate $g \Gamma_{2} g^{-1}$ of $\Gamma_{2}$ by an element $g \in \mathrm{PO}(n, 1)$.

In the correspondence of Theorem 2.15, commensurability of manifolds corresponds to commensurability of lattices. If one is interested in the study of hyperbolic manifolds up to commensurability, it is thus equivalent to study general lattices in $\mathrm{PO}(n, 1)$.

2.2.4 Subgroup separability. Replacing a lattice $\Gamma \subset \mathrm{PO}(n, 1)$ by a finite-index subgroup allows more than just removing torsion, as we will explain now.

Definition 2.2.11. Let $\Gamma$ be a group. The profinite topology of $\Gamma$ is the coarsest topology for which finite index subgroups $\Gamma^{\prime} \subset \Gamma$ and their cosets are open. A subgroup of $\Gamma$ is said to be separable in $\Gamma$ if it is closed in the profinite topology.

It follows from the definition that a finite index subgroup $\Gamma^{\prime}$ (and any of its cosets) is also closed. Thus, if $\Lambda \subset \Gamma$ is a subgroup, its closure in the profinite topology is given by

$$
\bar{\Lambda}=\bigcap_{\Lambda \subset \Gamma^{\prime} \subset_{\mathrm{fi}} \Gamma} \Gamma^{\prime}
$$


where the intersection is taken over all finite index subgroup of $\Gamma$ that contain $\Lambda$. The following lemma relates this to our situation:

Lemma 2.20. Let $\mathbf{G}$ be a real algebraic group and let $\mathbf{G}_{0} \subset \mathbf{G}$ be a real algebraic subgroup. Let $\Gamma \subset \mathbf{G}(\mathbb{R})$ be finitely generated. Then $\Gamma \cap \mathbf{G}_{0}(\mathbb{R})$ is separable in $\Gamma$.

Proof. This is the Lemme Principal in [Be00], slightly reformulated in our terminology.

The main application for us is the following:

Theorem 2.21. Let $\Gamma \subset \mathrm{PO}(n, 1)$ be a torsion-free lattice, and let $R \subset \mathbf{H}^{n}$ be a hyperplane. Suppose that $\Lambda=\operatorname{Stab}_{\Gamma}(R)$ is a lattice in $\operatorname{Stab}_{\mathrm{PO}(n, 1)}(R)$; let $N$ denote the corresponding manifold $\Lambda \backslash R$. Then there exists a finite cover $M^{\prime}$ of $M=\Gamma \backslash \mathbf{H}^{n}$ such that the inclusion $R \hookrightarrow \mathbf{H}^{n}$ induces an embedding $N \hookrightarrow M^{\prime}$.

Proof. This is Théorème 1' from [Be00]; we include a proof in the case where $N$ is compact. In that case, there exists a compact fundamental domain $D \subset R$ for the action of $\Lambda$. Since the action of $\Gamma$ on $\mathbf{H}^{n}$ is proper and $D$ is compact, the set $E=\{\gamma \in \Gamma \mid \gamma D \cap D \neq \emptyset\}$ is finite. Assume $E \backslash \Lambda=\left\{\gamma_{1}, \ldots, \gamma_{n}\right\}$. By Lemma 2.20, $\Lambda$ is separable in $\Gamma$. Hence for each $i$ there must exist a subgroup $\Gamma_{i} \subset \Gamma$ of finite index, containing $\Lambda$ but not $\gamma_{i}$. The intersection $\Gamma^{\prime}=\bigcap_{i} \Gamma_{i}$ is then still of finite index in $\Gamma$, and the manifold $\Gamma^{\prime} \backslash \mathbf{H}^{n}$ has the desired properties.

In the case where the lattice $\Gamma$ is arithmetic, we will be able to prove stronger separability results (see Section 2.3.4).

2.2.5 The hyperbolic models $\mathbf{H}_{f}$. In this section we will introduce a slightly modified version of the hyperboloid model of hyperbolic space, as it will play an important role in the rest of the thesis.

Let $k \subset \mathbb{R}$ be a number field, and let $f$ be a quadratic form defined over $k$. Assume that $f$ has signature $(n, 1)$ when seen over $\mathbb{R}$. Then the same procedure as above for the definition of $\mathbf{H}^{n}$ can be applied to the quadratic form $f$. More precisely, we define $H_{f}$ to be the level set $\left\{x \in \mathbb{R}^{n+1} \mid f(x)=-1\right\}$, and set $\mathbf{H}_{f}:=H_{f} /\{ \pm 1\}$. Similarly, the quadratic form $f$ induces a Riemannian metric $\langle\cdot, \cdot\rangle_{f}$ on tangent spaces which makes $\mathbf{H}_{f}$ into a Riemannian manifold.

Since $f$ has signature $(n, 1)$ there exists a matrix $A \in \mathrm{GL}_{n+1}(\mathbb{R})$ such that $f \circ A=f_{n, 1}=-x_{0}^{2}+x_{1}^{2}+\cdots+x_{n}^{2}$, the standard signature $(n, 1)$ quadratic form. This matrix induces an isometry $\mathbf{H}^{n} \rightarrow \mathbf{H}_{f}$ via $x \mapsto A x$, and thus $\mathbf{H}_{f}$ is also a model for the hyperbolic space.

The corresponding isometry group is easily seen to be:

$$
\operatorname{Isom}\left(\mathbf{H}_{f}\right) \cong \mathbf{P O}_{f}(\mathbb{R})
$$


the real points of the algebraic group $\mathbf{P O}_{f}$, with its natural action on $\mathbf{H}_{f}$. We will see in Section 3.2 that these models are particularly well suited to study manifolds which contain an immersed hypersurface.

The hyperplanes defined in Section 2.2.2 correspond here to $f$-orthogonal complements to vectors of $\mathbb{R}^{n+1}$ with positive $f$-norm. Explicitly, each hyperplane $R \subset H_{f}$ is obtained as

$$
R=\left(x^{\perp_{f}} \cap H_{f}\right) /\{ \pm 1\} \quad \text { for some } x \in \mathbb{R}^{n+1} \text { with } f(x)>0 .
$$

In this case however, the quadratic form $f$ endows $\mathbb{R}^{n+1}$ with a natural structure of quadratic space over $k$. Thus it makes sense to look at hyperplanes which come from vectors in $k^{n+1}$.

Definition 2.2.12. A hyperplane $R \subset \mathbf{H}_{f}$ is defined over $k$ if it corresponds to the $f$-orthogonal complement of some vector $x \in k^{n}$.

Equivalently, one may take $x \in \mathcal{O}_{k}^{n}$ by clearing the denominators, where $\mathcal{O}_{k}$ denotes the ring of integers of $k$.

\subsection{Arithmetic hyperbolic manifolds}

Arithmetic manifolds are the most basic examples of hyperbolic manifolds in arbitrary dimension. This class of manifolds is particularly well understood and plays a central role in the gluing constructions used to create nonarithmetic manifolds (see Chapter 4). We devote this section to them, and refer to [Bo69] as well as [Mo15] for a detailed introduction.

2.3.1 Definition of arithmetic lattices. In this section we will define arithmetic hyperbolic lattices and show that they are a natural generalization of the usual lattice $\mathbb{Z} \subset \mathbb{R}$. We will define them as lattices in $\mathrm{PO}(n, 1)$ the isometry group of the hyperbolic space $\mathbf{H}^{n}$, up to commensurability. We start with the easiest example and generalize it step by step until we reach the general definition.

Let $f_{n, 1}=-x_{0}^{2}+x_{1}^{2}+\cdots+x_{n}^{2}$ be the standard signature $(n, 1)$ quadratic form of Section 2.2. Then from the fact that $\mathbb{Z} \subset \mathbb{R}$ is discrete, it follows easily that the subgroup

$$
\mathbf{O}_{f}(\mathbb{Z}) \subset \mathbf{O}_{f}(\mathbb{R})
$$

is also discrete (here we see $\mathbf{O}_{f}$ as embedded in $\mathbf{G} \mathbf{L}_{n+1}$, see Example 2.1.13). The following theorem implies that it is also of finite covolume, hence a lattice in $\mathbf{O}_{f}(\mathbb{R})$. Since $\mathrm{PO}(n, 1) \cong \mathbf{O}_{f}(\mathbb{R}) /\{ \pm 1\}$, we naturally obtain a lattice in $\mathrm{PO}(n, 1)$.

Theorem 2.22 (Borel-Harish-Chandra). Let $\mathbf{G}$ be a semisimple linear algebraic $\mathbb{Q}$-group. Let $\Phi: \mathbf{G} \rightarrow \mathbf{G L}_{N}$ be a faithful algebraic representation, and set $\mathbf{G}(\mathbb{Z})=\Phi^{-1}\left(\mathbf{G L}_{N}(\mathbb{Z})\right)$. Then $\mathbf{G}(\mathbb{Z})$ is a lattice in $\mathbf{G}(\mathbb{R})$. 
See [BHC62, Section 7] for a proof. Observe that in the theorem, the subgroup $\mathbf{G}(\mathbb{Z})$ depends on the embedding of $\mathbf{G}$ into some $\mathbf{G L}_{N}$. It turns out that if one considers only the group $\mathbf{G}(\mathbb{Z})$ up to commensurability, then it becomes independent of the embedding. A proof of this fact can be found in [Bo69, Corollaire 7.13].

We will generalize this construction in three consecutive steps. First, observe that the same argument works for any quadratic form $f$ defined over $\mathbb{Q}$, provided it has signature $(n, 1)$. Indeed, these conditions are sufficient for $\mathbf{O}_{f}$ to be a semisimple algebraic $\mathbb{Q}$-group such that $\mathbf{O}_{f}(\mathbb{R}) /\{ \pm 1\} \cong \mathrm{PO}(n, 1)$, and thus $\mathbf{O}_{f}(\mathbb{Z})$ determines a lattice in $\operatorname{PO}(n, 1)$.

The second step is to replace $\mathbb{Q}$ with a number field $k$ and $\mathbb{Z}$ with its ring of algebraic integers $\mathcal{O}_{k}$. Here we need an apparently stronger version of the theorem of Borel and Harish-Chandra, stated for number fields:

Theorem 2.23 (Borel-Harish-Chandra for number fields). Let $\mathbf{G}$ be a semisimple linear algebraic k-group, for $k$ a number field. Let $\Phi: \mathbf{G} \rightarrow \mathbf{G L}_{N}$ be a faithful algebraic representation, and set $\mathbf{G}\left(\mathcal{O}_{k}\right)=\Phi^{-1}\left(\mathbf{G L}_{N}\left(\mathcal{O}_{k}\right)\right)$. Then $\mathbf{G}\left(\mathcal{O}_{k}\right)$ is a lattice in $\mathbf{G}\left(k \otimes_{\mathbb{Q}} \mathbb{R}\right)$.

Here we view $\mathbf{G}\left(\mathcal{O}_{k}\right)$ inside $\mathbf{G}\left(k \otimes_{\mathbb{Q}} \mathbb{R}\right)$ via the map induced by $k \hookrightarrow k \otimes_{\mathbb{Q}} \mathbb{R}$. Recall that $k \otimes_{\mathbb{Q}} \mathbb{R}$ is naturally isomorphic to the product

$$
k \otimes_{\mathbb{Q}} \mathbb{R} \cong \prod_{\sigma: k \hookrightarrow \mathbb{R}} \mathbb{R} \times \prod_{\{\sigma, \bar{\sigma}\}: k \hookrightarrow \mathbb{C}} \mathbb{C},
$$

so that

$$
\mathbf{G}\left(k \otimes_{\mathbb{Q}} \mathbb{R}\right) \cong \prod_{\sigma: k \hookrightarrow \mathbb{R}}{ }^{\sigma} \mathbf{G}(\mathbb{R}) \times \prod_{\{\sigma, \bar{\sigma}\}: k \hookrightarrow \mathbb{C}}{ }^{\sigma} \mathbf{G}(\mathbb{C}) .
$$

The inclusion $\mathbf{G}(k) \hookrightarrow \mathbf{G}\left(k \otimes_{\mathbb{Q}} \mathbb{R}\right)$ is then given by $x \mapsto(\sigma(x))_{\sigma}$.

While this theorem appears to be stronger, it reduces quite easily to the previous one using Weil's restriction of scalars, which we will not discuss here. We refer to [BHC62, Section 12] for a precise proof. Since restriction of scalars preserves commensurability of subgroups, we have (as before) that the group $\mathbf{G}\left(\mathcal{O}_{k}\right)$ is well defined (and does not depend on a representation into $\mathbf{G L}_{N}$ ) when considered up to commensurability.

This allows one to do a similar trick to obtain new lattices in $\mathrm{PO}(n, 1)$. Let $k \subset \mathbb{R}$ be a totally real-number field, and let $f$ be a quadratic form defined over $k$, having signature $(n, 1)$ and such that ${ }^{\sigma} f$ is positive definite for all other embeddings $\sigma: k \hookrightarrow \mathbb{R}$. Consider the group $\mathbf{P O}(\mathbb{R})$; since $f$ has signature $(n, 1)$, it is isomorphic to $\mathrm{PO}(n, 1)$. On the other hand, all conjugate $\operatorname{groups}^{\sigma}\left(\mathbf{P O}_{f}\right)(\mathbb{R})=\mathbf{P} \mathbf{O}_{f}(\mathbb{R})$ are compact, since ${ }^{\sigma} f$ is positive definite. Thus, applying the theorem to the group $\mathbf{P O}_{f}\left(\mathcal{O}_{k}\right)$, it follows that

$$
\mathbf{P O}_{f}\left(\mathcal{O}_{k}\right) \subset \mathbf{P O}_{f}\left(k \otimes_{\mathbb{Q}} \mathbb{R}\right)=\prod_{\sigma: k \hookrightarrow \mathbb{R}} \mathbf{P O}_{\sigma_{f}}(\mathbb{R}) \cong \mathrm{PO}(n, 1) \times \text { compact }
$$


is a lattice. Since modding out by a compact factor preserves both discreteness and finiteness of covolume, we have constructed a lattice in $\mathrm{PO}(n, 1)$.

Definition 2.3.1. A quadratic form $f$ over a totally real number field $k \subset \mathbb{R}$ is admissible (for $\mathrm{PO}(n, 1)$ ) if it has signature $(n, 1)$, and if ${ }^{\sigma} f$ is positive definite for all non-trivial embeddings $\sigma: k \hookrightarrow \mathbb{R}$.

The last and final generalization is to consider more general algebraic groups instead of restricting to ones of the form $\mathbf{P O} f$ for some quadratic form $f$. This leads us towards the following definition.

Definition 2.3.2. Let $\mathbf{G}$ be an algebraic $k$-group, with $k \subset \mathbb{R}$ be a number field. Then $\mathbf{G}$ is said to be admissible (for $\mathrm{PO}(n, 1)$ ) if $\mathbf{G}(\mathbb{R}) \cong \mathrm{PO}(n, 1)$ and if all the other factors in $\mathbf{G}\left(k \otimes_{\mathbb{Q}} \mathbb{R}\right)$ are compact. An arithmetic lattice in $\mathrm{PO}(n, 1)$ is a lattice which is commensurable to $\mathbf{G}\left(\mathcal{O}_{k}\right)$ for an admissible $\mathbf{G}$. An arithmetic manifold is a manifold $M=\Gamma \backslash \mathbf{H}^{n}$ where $\Gamma$ is an arithmetic lattice.

As explained before, the group $\mathbf{G}\left(\mathcal{O}_{k}\right)$ is well defined up to commensurability; thus the definition makes sense. Moreover it is clear that $\mathbf{P} \mathbf{O}_{f}$ is admissible if and only if $f$ is.

Remark 2.3.3. In order to produce arithmetic lattices in $\operatorname{PO}(n, 1)$, it would be enough to require an isomorphism $\mathbf{G}(\mathbb{R}) \cong \mathrm{PO}(n, 1)^{\circ}$ to the identity components of the Lie group $\mathrm{PO}(n, 1)$. It turns out that any such isomorphism is induced by an algebraic isomorphism $\mathbf{G}_{\mathbb{R}} \cong \mathbf{P O}_{(n, 1)}^{\circ}=\mathbf{P O}_{f_{n, 1}, \mathbb{R}}^{\circ}$ (see [Mi13]). Therefore, $\mathbf{G}$ is a $k$-form of $\mathbf{P O}_{(n, 1)}^{\circ}$, and thus a classical group of type $B_{\frac{n}{2}}$ or $D_{\frac{n+1}{2}}$ depending on the parity of $n$. By looking at the classification of classical groups (see Theorem 2.8 and the remark after that), one can thus list all possible algebraic groups $\mathbf{G}$ with this property. In particular, it follows from this classification (more precisely, from Theorem 25.12, 26.15 and 44.8 in [INV98]) that each such $\mathbf{G}$ is the identity component of an algebraic $k$-group $\mathbf{F}$ such that $\mathbf{F}_{\mathbb{R}} \cong \mathbf{P} \mathbf{O}_{(n, 1)}$. Hence the above definition is not restrictive, and we obtain all arithmetic lattices in $\mathrm{PO}(n, 1)$ that way.

The easiest of those $k$ forms are the groups $\mathbf{P O}$ for quadratic forms $f$ over number fields, whence the next definition.

Definition 2.3.4. An arithmetic lattice of type I (or of the first/simplest type) in $\mathrm{PO}(n, 1)$ is a lattice which is commensurable to $\mathbf{P O}_{f}\left(\mathcal{O}_{k}\right)$ for an admissible $f\left(\right.$ under an isomorphism $\left.\mathbf{P O}_{f}(\mathbb{R}) \cong \mathrm{PO}(n, 1)\right)$.

We will need special type of subgroups of $\mathbf{G}\left(\mathcal{O}_{k}\right)$ called congruence subgroups. Let $\mathbf{G}$ be a semisimple linear algebraic group, and $\Phi: \mathbf{G} \rightarrow \mathbf{G L}_{N}$ a faithful algebraic representation. Let $\Gamma \subset \mathbf{G}$ be an arithmetic group that is commensurable to $\Phi^{-1}\left(\mathbf{G L}_{N}\left(\mathcal{O}_{k}\right)\right)$. Then we can construct finite index subgroups of $\Gamma$ as follows. For an ideal $\mathfrak{a} \subset \mathcal{O}_{k}$ the group $\operatorname{GL}_{N}\left(\mathcal{O}_{k} / \mathfrak{a}\right)$ is finite. 
Thus the kernel of the map $\operatorname{GL}_{N}\left(\mathcal{O}_{k}\right) \rightarrow \mathbf{G L}_{N}\left(\mathcal{O}_{k} / \mathfrak{a}\right)$ has finite index in $\mathbf{G L}_{N}\left(\mathcal{O}_{k}\right)$.

Definition 2.3.5. In the above setting, the group

$$
\Gamma(\mathfrak{a})=\Gamma \cap \Phi^{-1}\left(\operatorname{ker}\left(\mathbf{G L}_{N}\left(\mathcal{O}_{k}\right) \rightarrow \mathbf{G L}_{N}\left(\mathcal{O}_{k} / \mathfrak{a}\right)\right)\right)
$$

is called a (principal) congruence subgroup of $\Gamma$.

2.3.2 Examples. Here we regroup some important examples of arithmetic lattices which we will use later on.

Example 2.3.6. Let $f=-x_{0}^{2}+x_{1}^{2}+\cdots+x_{n}^{2}$. Then the image of the group $\mathbf{O}_{f}(\mathbb{Z})$ in $\mathbf{P O}_{f}(\mathbb{R}) \cong \mathrm{PO}(n, 1)$ is an arithmetic lattice there. By varying the coefficients of $f$ one can construct different (that is, non-commensurable) arithmetic groups in $\mathrm{PO}(n, 1)$. This is made precise in the next Theorem.

Theorem 2.24. For $i=1,2$, let $\Gamma_{i} \subset \mathrm{PO}(n, 1)$ be an arithmetic manifold of type $I$, coming from a quadratic form $f_{i}$. Then $\Gamma_{1}$ and $\Gamma_{2}$ are commensurable if and only if $f_{1}$ and $f_{2}$ are similar.

Proof. This is proven in [GPS87, §2.6], and in [Me17, Proposition 5.4]. We include a short proof (in dimension $n \geq 4$ ) which makes use of our machinery. By Theorem 2.14, the quadratic forms $f_{1}$ and $f_{2}$ are similar if and only if the corresponding algebraic groups $\mathbf{P} \mathbf{O}_{f_{1}}$ and $\mathbf{P} \mathbf{O}_{f_{2}}$ are isomorphic. If the groups are isomorphic, since the commensurability class defined by $\mathbf{P O}_{f_{i}}\left(\mathcal{O}_{k}\right)$ is independent of the embedding in some $\mathbf{G L}_{N}$ (and hence depends only on the isomorphism type of $\mathbf{P} \mathbf{O}_{f_{i}}$ ), we see that the groups $\Gamma_{1}$ and $\Gamma_{2}$ are commensurable. Conversely, if they are commensurable, we can assume (up to changing the isomorphism $\left.\mathbf{P O}_{f_{1}}(\mathbb{R}) \cong \mathrm{PO}(n, 1)\right)$ that $\Gamma=\Gamma_{1} \cap \Gamma_{2}$ is of finite index both in

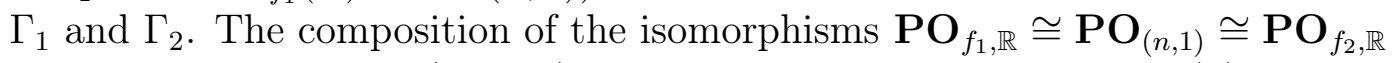
then sends $\Gamma$ which is (almost) Zariski-dense in $\mathbf{P} \mathbf{O}_{f_{1}}$ into $\mathbf{P} \mathbf{O}_{f_{2}}(k)$. Hence the isomorphism is induced by a $k$-isomorphism $\mathbf{P O}_{f_{1}} \cong \mathbf{P O}_{f_{2}}$ by Proposition 2.13 .

Example 2.3.7. Using this theorem, one can easily construct examples of non-commensurable arithmetic manifolds. Indeed, let $n$ be odd, and consider the quadratic forms

$$
f_{a}=-x_{0}^{2}+x_{1}^{2}+\cdots+x_{n-1}^{2}+a x_{n}^{2} .
$$

Since the discriminant of $\lambda f_{a}$ equals $a \bmod \left(\mathbb{Q}^{\times}\right)^{2}$ for any $\lambda \in \mathbb{Q}^{\times}$, we see that two forms $f_{a}$ and $f_{b}$ are similar if and only if $a \equiv b \bmod \left(\mathbb{Q}^{\times}\right)^{2}$. In that case the forms are even isometric.

It follows that, for instance, the arithmetic groups $\mathbf{O}_{f_{p}}(\mathbb{Z})$ for primes $p$ all lead to pairwise non-commensurable arithmetic lattices in $\mathrm{PO}(n, 1)$. For even dimensions, more care is needed to obtain non-similar quadratic forms (compare Lemma 4.16). However, it is still possible to construct interesting nonarithmetic manifolds using similar quadratic forms, see Section 4.5.2. 
Example 2.3.8. As explained before, one can construct an arithmetic group starting from any $k$-form of the algebraic group $\mathbf{P} \mathbf{O}_{(n, 1)}$. If $n$ is odd, then $\mathbf{P O}_{(n, 1)}$ is of type $D_{m}$ where $m=\frac{n-1}{2}$ and thus there is a $k$-form given by groups of the form $\mathbf{P U}(D, h)$ where $D$ is a quaternion algebra over $k$ and $h$ is a skew-Hermitian form of dimension $m$ (see Theorem 2.8). As an example, one can take $k=\mathbb{Q}, D$ to be the quaternion algebra over $\mathbb{Q}$ generated by $1, i, j, i j$ such that $i^{2}=2, j^{2}=3$ and $i j=-j i$ and $h$ to be the skew-hermitian form on $D^{n}$ given by

$$
(x, y) \longmapsto \bar{x}_{1} \cdot i \cdot y_{1}+\bar{x}_{2} \cdot j \cdot y_{2}+\cdots+\bar{x}_{n} \cdot j \cdot y_{n}
$$

It can be shown that over $\mathbb{Q}(\sqrt{2}), \mathbf{P U}(D, \bar{x} \cdot j \cdot y)$ is isomorphic to $\mathbf{P O}$ where $f=x^{2}+3 y^{2}$, while $\mathbf{P U}(D, \bar{x} \cdot i \cdot y)$ is isomorphic to $\mathbf{P O}$ with $f=x^{2}-y^{2}$. All in all, we have

$$
\mathbf{P U}(D, h)_{\mathbb{Q}(\sqrt{2})} \cong \mathbf{P O}_{f, \mathbb{Q}(\sqrt{2})},
$$

where $f=-x_{0}^{2}+x_{1}^{2}+x_{2}^{2}+3 x_{3}^{2}+\cdots+x_{n-1}^{2}+3 x_{n}^{2}$. Thus $\mathbf{P} \mathbf{U}(D, h)$ is a $k$-form of $\mathbf{P O} \mathbf{O}_{(n, 1)}$, and the group $\mathbf{P U}(D, h)\left(\mathcal{O}_{k}\right)$ defines a lattice in $\mathrm{PO}(n, 1)$ (up to commensurability).

2.3.3 Hypersurfaces in arithmetic manifolds. In this section we will study hypersurfaces in arithmetic hyperbolic manifolds of type I. As we will see in Section 3.2, this is no restriction, as other arithmetic groups do not have hypersurfaces. More precisely, we will explain how to construct as many disjoint hypersurfaces in arithmetic manifolds of type I as we want. This will allow us to obtain pieces of manifolds with interesting properties.

Let $M=\Gamma \backslash \mathbf{H}^{n}$ be an arithmetic manifold of type I. By definition, there exists a totally real number field $k \subset \mathbb{R}$ and an admissible quadratic form $f$ over $k$ such that $\Gamma$ is commensurable to $\mathbf{P O}_{f}\left(\mathcal{O}_{k}\right)$. It will be convenient to use the hyperbolic model $\mathbf{H}_{f}$ defined by $f$ (see Section 2.2.5) with isometry group $\mathbf{P O}_{f}(\mathbb{R})$. More precisely, we will assume that $\Gamma \subset \mathbf{P O}_{f}(\mathbb{R})$ is commensurable to $\mathbf{P O}_{f}\left(\mathcal{O}_{k}\right)$ and that $M=\Gamma \backslash \mathbf{H}_{f}$.

Remark 2.3.9. It will follow from Theorem 3.13 that $\Gamma$ actually lies in $\mathbf{P O}_{f}(k)$, though we will not use this here.

Let $R \subset \mathbf{H}_{f}$ be a hyperplane which is defined over $k$ (see Definition 2.2.12), that is, $R=\left(x^{\perp_{f}} \cap H_{f}\right) /\{ \pm 1\}$ for some $x \in k^{n}$ (or equivalently, $x \in \mathcal{O}_{k}^{n}$ ). Let $V$ denote the quadratic space $\left(k^{n+1}, f\right)$, and let $W$ denote the subspace corresponding to $R$ (i.e., $W=x^{\perp_{f}} \subset V$ ). Choose an orthogonal basis for $W$ and extend it to $V$. The change of base matrix $A \in \mathrm{GL}_{n+1}(k)$ induces an isometry between $f$ and

$$
f^{\prime}=f \circ A^{-1}=a_{0} x_{0}^{2}+\cdots+a_{n} x^{2} .
$$


This matrix $A$ thus induces an isometry

$$
\begin{aligned}
\phi: \mathbf{H}_{f} & \rightarrow \mathbf{H}_{f^{\prime}} \\
x & \mapsto A x
\end{aligned}
$$

sending $R$ to the hyperplane $\left\{x_{n}=0\right\} \cap H_{f^{\prime}} /\{ \pm 1\} \subset \mathbf{H}_{f^{\prime}}$. Finally, it induces via conjugation an isomorphism of the isometry groups

$$
\begin{aligned}
\Phi: \mathbf{P O}_{f} & \rightarrow \mathbf{P O}_{f^{\prime}} \\
g & \mapsto \phi^{-1} g \phi .
\end{aligned}
$$

Since $\Gamma$ is commensurable to $\mathbf{P O}_{f}\left(\mathcal{O}_{k}\right)$, and since the latter is independent (up to commensurability) of the embedding of $\mathbf{P O} \mathbf{O}_{f}$ into some $\mathbf{G L}_{N}$ (see Section 2.3.1) we have that $\Phi(\Gamma)$ is commensurable to $\mathbf{P O}_{f^{\prime}}\left(\mathcal{O}_{k}\right)$. It follows that

$$
\Phi\left(\operatorname{Stab}_{\Gamma}(R)\right)=\operatorname{Stab}_{\Phi(\Gamma)}(\phi(R))
$$

is commensurable to the subgroup of matrices of $\mathbf{P O}_{f^{\prime}}\left(\mathcal{O}_{k}\right)$ preserving the subspace $\left\{x_{n}=0\right\}$. The latter is commensurable to the subgroup $\mathbf{O}_{f_{0}^{\prime}}\left(\mathcal{O}_{k}\right)$ diagonally embedded in $\mathbf{P O}_{f^{\prime}}$, where $f_{0}^{\prime}$ is the quadratic form $f^{\prime}$ with last coordinate set to 0 . This is a lattice in $\mathbf{O}_{f_{0}^{\prime}}(\mathbb{R})$, by the Theorem of Borel and Harish-Chandra (Theorem 2.23).

Coming back to our original manifold $M=\Gamma \backslash \mathbf{H}_{f}$, we have shown that

$$
\operatorname{Stab}_{\Gamma}(R) \subset \operatorname{Stab}_{\mathbf{P O}_{f}(\mathbb{R})}(R)
$$

is a lattice, and it follows that $R$ projects down to an immersed hypersurface in $M=\Gamma \subset \mathbf{H}_{f}$.

Therefore, by subgroup separability (Theorem 2.21) we can find a subgroup $\Gamma_{1} \subset \Gamma$ of finite index such that the hyperplane $R$ maps onto an embedded hypersurface $N \subset M_{1}=\Gamma_{1} \backslash \mathbf{H}_{f}$. The hypersurface $N$ is then itself an arithmetic manifold, whose lattice is commensurable to $\mathbf{P O}_{f_{0}^{\prime}}\left(\mathcal{O}_{k}\right)$ (via the isomorphism $\Phi)$.

It will follow from Proposition 3.11 in Section 3.2.2 that the opposite direction is still true, i.e., that all hypersurfaces in arithmetic manifolds arise in that way:

Theorem 2.25. Let $M=\Gamma \backslash \mathbf{H}_{f}$ be an arithmetic manifold of type $I$, with $\Gamma$ commensurable to $\mathbf{P O}_{f}\left(\mathcal{O}_{k}\right)$.

1. Any hyperplane $R \subset \mathbf{H}_{f}$ that is defined over $k$ (i.e., $R=\left(x_{f}^{\perp} \cap H_{f}\right) /\{ \pm 1\}$ for some $x \in k^{n}$ ) projects onto an immersed hypersurface $N \subset M$.

2. Any hypersurface $N \subset M$ is the image of a hyperplane $R \subset \mathbf{H}_{f}$ that is defined over $k$. 
3. Any hypersurface $N \subset M$ is arithmetic, and commensurable to the arithmetic manifold defined by $\mathbf{P O}_{f_{0}}\left(\mathcal{O}_{k}\right) \backslash \mathbf{H}_{f_{0}}$, where $f_{0}$ is the quadratic form $f$ restricted to the subspace defined by $R$.

This construction gives a way to produce arithmetic manifolds containing prescribed hypersurfaces (up to commensurability).

Example 2.3.10. The quadratic forms $f_{a}$ from Example 2.3 .7 are all of the form

$$
f_{a}=f_{0}+a x_{n}^{2} \quad \text { where } f_{0}=-x_{0}^{2}+x_{1}^{2}+\cdots+x_{n-1}^{2} .
$$

Let $\Gamma_{a} \subset \mathbf{P O}_{f_{a}}\left(\mathcal{O}_{k}\right)$ be a torsion-free subgroup. It follows that the hyperplane in $\mathbf{H}_{f_{a}}$ corresponding to $\left\{x_{n}=0\right\}$ projects to an immersed hypersurface in the manifold $\Gamma_{a} \backslash \mathbf{H}_{f_{a}}$. This manifold is arithmetic and commensurable to $\mathbf{P O}_{f_{0}}\left(\mathcal{O}_{k}\right) \backslash \mathbf{H}_{f_{0}}$. This method is useful to construct non-commensurable arithmetic manifolds which contain commensurable hypersurfaces, as we will see in Section 4.2 .

2.3.4 Subgroup separability in arithmetic lattices. Subgroup separability (Theorem 2.21) is a useful tool for general lattices to pass from immersed hypersurfaces to embedded ones in a finite covering. In the case where the lattice is arithmetic, there are two important refinements of this principle which we explore now. The first result (due to Belolipetsky and Thomson [BT11]) gives a way to construct arithmetic manifolds with many disjoint hypersurfaces.

Theorem 2.26. Let $R_{1}, \ldots, R_{s} \subset \mathbf{H}_{f}$ be disjoint hyperplanes defined over $k$. Let $M=\Gamma \backslash \mathbf{H}_{f}$ be an arithmetic manifold of type $I$, with $\Gamma$ commensurable to $\mathbf{P O}_{f}\left(\mathcal{O}_{k}\right)$. Then there exists a finite index subgroup $\Gamma_{1} \subset \Gamma$ such that the hyperplanes $R_{i}$ project down to disjoint embedded hypersurfaces in the manifold $M_{1}=\Gamma_{1} \backslash \mathbf{H}_{f}$.

Proof. Lemma 3.1 in [BT11].

From the proof of this lemma, it follows that one can take $\Gamma_{1}$ to be a congruence subgroup $\Gamma(\mathfrak{a})$ of $\Gamma$. Moreover, as soon as the ideal $\mathfrak{a}$ does not contain 2 , the corresponding manifold $M_{1}$ and all the hypersurfaces will be orientable.

Another improvement in the case of arithmetic lattices concerns its geometrically finite subgroups (see [Ra06, §12.4]).

Definition 2.3.11. A discrete subgroup $\Gamma \subset \mathrm{PO}(n, 1)$ is geometrically finite $\left(\right.$ in $\mathbf{H}^{n}$ ) if it admits a fundamental domain $F \subset \mathbf{H}^{n}$ such that:

1. $F$ is a convex polyhedron,

2. Every side of $F$ is the intersection of $F$ with a translate $\gamma F$ of $F$, for some $\gamma \in \Gamma$, 
3. Every point $x \in \bar{F} \cap \partial \mathbf{H}^{n}$ admits a neighborhood $U$ such that all sides of $F$ meeting $U$ pass through $x$.

The main examples of geometrically finite subgroups that will interest us are the following:

\section{Theorem 2.27.}

1. Any torsion-free lattice $\Gamma \subset \mathrm{PO}(n, 1)$ is geometrically finite.

2. Any torsion-free lattice $\Gamma \subset \mathrm{O}(n-1,1) \cong \operatorname{Stab}_{\mathrm{PO}(n, 1)}(R)$ for a hyperplane $R \subset \mathbf{H}^{n}$ is geometrically finite in $\mathbf{H}^{n}$.

Proof. 1. is Theorem 12.7.3 of [Ra06]. 2. follows from 1: A fundamental poly-

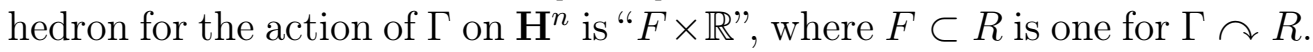

As seen in Section 2.2.4, for a lattice $\Gamma \subset \operatorname{PO}(n, 1)$, groups of the form $\operatorname{Stab}_{\Gamma}(R)$ are separable in $\Gamma$ (for any hyperplane $R \subset \mathbf{H}^{n}$ ). When $\Gamma$ is arithmetic of type I, this result extends to the more general case of geometrically finite subgroups:

Theorem 2.28. Let $\Gamma \subset \mathbf{P O}_{f}$ be an arithmetic lattice of type $I$. Then any geometrically finite subgroup of $\Gamma$ is separable (in $\Gamma$ ).

Proof. This is [BHW11, Corollary 1.12].

This can be used to construct arithmetic manifolds that contain a hypersurface isometric to a fixed arithmetic manifold, see [KRS18]. For us, it will be useful in Section 4.5.2 when we consider specific examples of manifolds obtained by "closing up" arithmetic pieces. 


\section{Chapter $3 \mid$ Trace Fields}

In this chapter we introduce the (adjoint) trace field of lattices in $\mathrm{PO}(n, 1)$, and the associated algebraic group called the ambient group. After defining these invariants in their various flavors (first for Zariski-dense subgroups, then for lattices and finally for monodromy representations of manifolds with boundary) we proceed towards the classification of ambient groups of manifolds which contain an immersed hypersurface. These will be especially useful in the gluing constructions of Chapter 4. Finally, we end this chapter by computing the trace field and ambient group of an arithmetic lattice and show that it coincides with its field and group of definition, respectively.

As the previous one, this chapter contains only standard material, and no substantially new results are presented.

\subsection{Trace fields and ambient groups}

This section is devoted to the various definitions of the trace fields we will need. We will first define them for Zariski-dense subgroups of general semisimple groups, then for lattices in $\mathrm{PO}(n, 1)$ and finally for monodromy representations of manifolds with boundary.

3.1.1 Definition for Zariski-dense subgroups. The trace field was introduced by Vinberg [Vi71] for general Zariski-dense subgroups of (not necessarily connected) semisimple algebraic groups, as follows. Recall that for an algebraic group $\mathbf{G}$ we have the adjoint representation $A d: \mathbf{G} \rightarrow \mathbf{G L}(\mathfrak{g})$, where $\mathfrak{g}$ is the Lie algebra of $\mathbf{G}$.

Definition 3.1.1. Let $\mathbf{G}$ be a semisimple algebraic group, and $\Gamma \subset \mathbf{G}$ a Zariski-dense subgroup. The (adjoint) trace field of $\Gamma$ is the field

$$
\mathbb{Q}(\operatorname{tr} \operatorname{Ad} \gamma \mid \gamma \in \Gamma)
$$

The goal in the remaining of this section is to convince the reader that this adjoint trace field is a sensible invariant which applies very well to the study of hyperbolic manifolds. First we will show that if the group is adjoint, this 
field can be seen as the smallest field over which the group $\Gamma$ can be realized. The following theorem was first (implicitly) proven by Vinberg [Vi71]. Two other proofs can be found in [DM86] and in [Ma91]. We will give a proof (in the case of absolutely simple groups) which is a mixture of both.

Theorem 3.1. Let $\mathbf{G}$ be an adjoint semisimple algebraic group and $\Gamma \subset \mathbf{G}$ a Zariski-dense subgroup. Let $k$ be the trace field of $\Gamma$. Then there exists an algebraic k-group $\mathbf{F}$ and an isomorphism $\Phi: \mathbf{G} \rightarrow \mathbf{F}_{\mathbb{C}}$ such that $\Phi(\Gamma) \subset \mathbf{F}(k)$.

Proof. It is enough to find a faithful algebraic representation $\Phi: \mathbf{G} \rightarrow \mathbf{G L}(W)_{\mathbb{C}}$ with $\Phi(\Gamma) \subset \mathbf{G L}(W)(k)$, for some finite-dimensional $k$-vector space $W$. Indeed, the Zariski-closure $\mathbf{F}$ of $\Phi(\Gamma)$ in $\mathbf{G L}(W)$ then forms a $k$-group, and $\Phi$ induces an isomorphism $\mathbf{G} \cong \mathbf{F}_{\mathbb{C}}$.

We will assume that $\mathbf{G}$ is absolutely simple (see [Vi71] for the general case). Write $\widehat{\cdot}$ for Ad. Let $\mathfrak{g}$ denote the Lie algebra of $\mathbf{G}$, seen as a vector space over $\mathbb{C}$. Let $V$ (resp. $W$ ) denote the $\mathbb{C}$-span (resp. the $k$-span) of the set $\widehat{\Gamma}=\operatorname{Ad} \Gamma \subset \operatorname{End}_{\mathbb{C}}(\mathfrak{g})$. Since $\mathbf{G}$ is simple, so is $\mathfrak{g}$ and thus the adjoint representation is irreducible. By Burnside's theorem on irreducible representations [Wa91, §14.8] we have that $V=\operatorname{End}_{\mathbb{C}}(\mathfrak{g})$.

Let $\widehat{\gamma}_{1}, \ldots, \widehat{\gamma}_{m} \in \widehat{\Gamma}$ be a $\mathbb{C}$-basis of $V$. Let $e_{1}, \ldots, e_{m}$ be a dual basis with respect to the bilinear form $(x, y)=\operatorname{tr}(x y)$. It is easy to see that for all $x \in V$, we have $x=\sum_{i} \operatorname{tr}\left(\widehat{\gamma}_{i} x\right) e_{i}$. It follows that for any $\gamma \in \Gamma$, we have $\widehat{\gamma}=\sum_{i} \operatorname{tr}\left(\widehat{\gamma}_{i} \widehat{\gamma}\right) e_{i}$, so that $\widehat{\gamma}$ is in the $k$-span of $\left\{e_{1}, \ldots, e_{n}\right\}$.

Thus $W$ is a finite-dimensional $k$-vector space such that $W_{\mathbb{C}}=V$. The action of $\mathbf{G}$ on $\mathfrak{g}$ induces an algebraic action of $\mathbf{G}$ on $V=\operatorname{End}_{\mathbb{C}}(\mathfrak{g})$ and thus an algebraic representation $\Phi: \mathbf{G} \rightarrow \mathbf{G L}(V)=\mathbf{G L}(W)_{\mathbb{C}}$ which is faithful since $\mathbf{G}$ is adjoint. Since $\widehat{\gamma} \cdot W \subset W$, we have $\Phi(\Gamma) \subset \mathbf{G L}(W)(k)$, as desired.

From the Zariski-density of $\Gamma$ we obtain the first corollary:

Corollary 3.2 (Uniqueness of $\mathbf{F}$ ). The algebraic group $\mathbf{F}$ is unique up to k-isomorphism.

Proof. Let $\mathbf{F}^{\prime}$ be another algebraic $k$-group with $\Phi^{\prime}: \mathbf{G} \rightarrow \mathbf{F}_{\mathbb{C}}^{\prime}$ an isomorphism such that $\Phi^{\prime}(\Gamma) \subset \mathbf{F}^{\prime}(k)$. Then the isomorphism $\Phi^{\prime} \circ \Phi^{-1}: \mathbf{F}_{\mathbb{C}} \rightarrow \mathbf{F}_{\mathbb{C}}^{\prime}$ sends the Zariski-dense subgroup $\Phi(\Gamma) \subset \mathbf{F}(k)$ into $\mathbf{F}^{\prime}(k)$. Thus Corollary 2.2 implies that it is defined over $k$.

In view of this, we make the following definition:

Definition 3.1.2. Let $\mathbf{G}$ be an adjoint semisimple algebraic group and $\Gamma \subset \mathbf{G}$ a Zariski-dense subgroup. Let $k$ be the trace field of $\Gamma$. Then the ambient group of $\Gamma$ is the unique algebraic $k$-group $\mathbf{F}$ (up to $k$-isomorphism) such that $\mathbf{F}_{\mathbb{C}} \cong \mathbf{G}$ and $\Gamma \subset \mathbf{F}(k)$ via this isomorphism.

The trace field can also be seen as the minimal field over which $\Gamma$ can be realized: 
Corollary 3.3 (Minimality of $k$ ). The trace field of $\Gamma$ is the minimal field $k$ such that the conclusion of Theorem 3.1 holds.

Proof. Let $k$ be a field and $\mathbf{F}$ an algebraic $k$-group with an isomorphism $\Phi: \mathbf{G} \rightarrow \mathbf{F}_{\mathbb{C}}$ such that $\Phi(\Gamma) \subset \mathbf{F}(k)$. Let $\mathfrak{g}$ be the Lie algebra of $\mathbf{G}$ and $\mathfrak{f}$ that of $\mathbf{F}$. Since $\Phi$ is an isomorphism, it induces an isomorphism of $\mathbb{C}$-vector spaces $\mathfrak{g} \cong \mathfrak{f} \otimes \mathbb{C}$. Since $\Phi(\Gamma) \subset \mathbf{F}(k)$, we have $\operatorname{Ad} \Phi(\Gamma) \subset \operatorname{Ad} \mathbf{F}(k)$. Thus there exists a basis of $\mathfrak{g}$ (the one coming from $\mathfrak{f}$ ) such that the matrices of $\operatorname{Ad} \Gamma$ have all coefficients in $k$. Therefore, the trace field of $\Gamma$ is contained in $k$.

Finally, the third corollary tells us that the trace field is invariant under commensurability.

Corollary 3.4. Let $\Gamma, \Gamma^{\prime} \subset \mathbf{G}$ be Zariski-dense subgroups of an adjoint semisimple algebraic group which are commensurable (i.e., there is a $g \in \mathbf{G}$ such that $\Gamma \cap g \Gamma^{\prime} g^{-1}$ has finite index both in $\Gamma$ and in $\left.g \Gamma^{\prime} g^{-1}\right)$. Then the trace field of $\Gamma$ equals that of $\Gamma^{\prime}$.

Proof. Since the trace is invariant under conjugation, it is enough to show that if $\Gamma_{0} \subset \Gamma$ is a finite index subgroup, then the trace fields of $\Gamma$ and $\Gamma_{0}$ coincide. Clearly, the trace field of $\Gamma_{0}$ is included in that of $\Gamma$. For the reverse inclusion, we can (up to replacing $\Gamma_{0}$ with a further finite index subgroup) assume that $\Gamma_{0}$ is normal in $\Gamma$, and that $\Gamma_{0} \subset \mathbf{G}^{\circ}$. From the proof of Theorem 3.1, we find a $k_{0}$-vector space $W$ and a faithful representation $\Phi: \mathbf{G} \rightarrow \mathbf{G L}\left(W_{\mathbb{C}}\right)$ such that $\Phi\left(\Gamma_{0}\right) \subset \mathbf{G L}(W)\left(k_{0}\right)$, where $k_{0}$ is the trace field of $\Gamma_{0}$. We identify $\mathbf{G}$ with $\Phi(\mathbf{G})$, so that $\Gamma_{0} \subset \mathbf{G}\left(k_{0}\right)$. Our goal is now to show that actually $\Gamma \subset \mathbf{G}\left(k_{0}\right)$.

Let $\sigma$ be an automorphism of $\mathbb{C}$ fixing $k_{0}$. It is enough to show that $\sigma$ fixes all elements of $\Gamma$. For such $\gamma \in \Gamma$ we have $\gamma \Gamma_{0} \gamma^{-1}=\Gamma_{0}$, and thus for any $\gamma_{0} \in \Gamma_{0}$,

$$
\sigma(\gamma) \gamma_{0} \sigma\left(\gamma^{-1}\right)=\gamma \gamma_{0} \gamma^{-1}
$$

It follows that $\gamma^{-1} \sigma(\gamma)$ commutes with every element of $\Gamma_{0}$. By Zariski-density, $\gamma^{-1} \sigma(\gamma)$ is in $\mathbf{Z}_{\mathbf{G}}\left(\mathbf{G}^{\circ}\right)$, the centralizer of $\mathbf{G}^{\circ}$ in $\mathbf{G}$. Since $\mathbf{G}$ is adjoint, this centralizer is trivial (by Proposition 2.7), and we have $\sigma(\gamma)=\gamma$.

3.1.2 Definition for lattices. Let $\Gamma$ be a lattice in the real Lie group $\mathrm{PO}(n, 1)$. We wish to define a notion of adjoint trace field and ambient group for $\Gamma$. For the trace field, we can use the adjoint representation Ad for Lie groups to make the following analog definition:

Definition 3.1.3. Let $\Gamma \subset \mathrm{PO}(n, 1)$ be a lattice. Then the (adjoint) trace field of $\Gamma$ is the field

$$
\mathbb{Q}(\operatorname{tr} \operatorname{Ad}(\gamma) \mid \gamma \in \Gamma) .
$$

The trace field of a manifold $M=\Gamma \backslash \mathbf{H}^{n}$ is the trace field of its corresponding lattice $\Gamma$. 
Remark 3.1.4. It follows that the trace field of a manifold is always naturally embedded in $\mathbb{R}$. This is in contrast with the invariant trace field, usually studied in the context of 3-manifolds, see [MR03, Chapter 3]. It can be defined as in Definition 3.1.3, but using the complex Lie group $\mathbf{P S L}_{2}(\mathbb{C})$ as a model for the (orientation preserving) isometries of $\mathbf{H}^{3}$ and its corresponding complex adjoint representation [MR03, Exercise 3.3.4]. In that case, the invariant trace field of a lattice is never real [MR03, Theorem 3.3.7].

As seen in Section 2.2.1, the Lie group $\operatorname{PO}(n, 1)$ has a natural structure of real algebraic group: for $f_{n, 1}=-x_{0}^{2}+x_{1}^{2}+\cdots+x_{n}^{2}$ the algebraic $\mathbb{R}$-group $\mathbf{P O} \mathbf{O}_{(n, 1)}=\mathbf{P} \mathbf{O}_{f_{n, 1}}$ is such that $\mathbf{P O} \mathbf{O}_{(n, 1)}(\mathbb{R}) \cong \mathrm{PO}(n, 1)$. Recall that $\mathbf{P} \mathbf{O}_{(n, 1)}$ is connected for $n$ even, and has two connected components for $n$ odd. We let $\mathbf{P O}_{(n, 1)}^{\circ}$ denote its identity component. The following theorem allows us to relate the two notions of trace fields (see [Mo15, §4.5]).

Theorem 3.5 (Borel's Density Theorem). Any lattice $\Gamma \subset \mathbf{P O}_{(n, 1)}^{\circ}(\mathbb{R})$ is Zariski-dense in $\mathbf{P O}_{(n, 1)}^{\circ}$.

It follows that for a lattice $\Gamma \subset \mathrm{PO}(n, 1)$ the following situations can occur:

1. $n$ is even or $\Gamma \not \subset \mathbf{P O}_{(n, 1)}^{\circ}(\mathbb{R})$ : In that case, we have that the Zariskiclosure of $\Gamma$ is the whole algebraic group $\mathbf{P O}_{(n, 1)}$.

2. $n$ is odd and $\Gamma \subset \mathbf{P O}_{(n, 1)}^{\circ}(\mathbb{R})$ : In that case, the Zariski-closure of $\Gamma$ is not $\mathbf{P O}_{(n, 1)}$ but just its identity component $\mathbf{P} \mathbf{O}_{(n, 1)}^{\circ}$. We will see soon how to deal with this possibility.

Thus we can make the following definition, compatible with Definition 3.1.2:

Definition 3.1.5. Let $\Gamma \subset \mathrm{PO}(n, 1)=\mathbf{P O}_{(n, 1)}(\mathbb{R})$ be a lattice with trace field $k$. Then the ambient group of $\Gamma$ is the unique algebraic $k$-group $\mathbf{G}$ (up to $k$ isomorphism) such that $\mathbf{G}_{\mathbb{R}}$ is isomorphic to the Zariski-closure of $\Gamma$ in $\mathbf{P O}_{(n, 1)}$ (that is, $\mathbf{P O}_{(n, 1)}$ or $\left.\mathbf{P} \mathbf{O}_{(n, 1)}^{\circ}\right)$ and $\Gamma \subset \mathbf{G}(k)$ via this isomorphism. The ambient group of a manifold $M=\Gamma \backslash \mathbf{H}^{n}$ is the ambient group of its corresponding lattice.

Remark 3.1.6. It follows that $\mathbf{G}^{\circ}$ is a $k$-form of the algebraic $\mathbb{R}$-group $\mathbf{P O}_{(n, 1)}^{\circ}$. Now in the case where $n=2 m+1$ is odd, $\mathbf{P O}_{(n, 1)}^{\circ}$ is an adjoint group of type $D_{m}$. As explained in Remark 2.3.3, it follows from the classification of those groups that $\mathbf{G}^{\circ}$ is the identity component of some algebraic $k$-group $\mathbf{G}$ such that $\mathbf{G}_{\mathbb{R}} \cong \mathbf{P O}(n, 1)$. Therefore, even in the case where $\bar{\Gamma}=\mathbf{P} \mathbf{O}_{(n, 1)}^{\circ} \neq \mathbf{P} \mathbf{O}_{(n, 1)}$, we can still find an algebraic $k$-group $\mathbf{G}$ such that $\mathbf{G}_{\mathbb{R}} \cong \mathbf{P} \mathbf{O}_{(n, 1)}$, and $\Gamma \subset \mathbf{G}(k)$ via this isomorphism. By abuse of notation, we will again call $\mathbf{G}$ the ambient group of $\Gamma$. Note finally that these considerations will play little role in the later parts of this thesis, since all our algebraic groups will be of the form $\mathbf{P O}_{f}$ for some quadratic form $f$ (see Theorem 3.8 below). 
We will end this section by stating the most important theorem about the trace field of lattices.

Theorem 3.6. Let $\Gamma \subset \mathrm{PO}(n, 1)$ be a lattice. If $n \geq 3$, then the trace field of $\Gamma$ is a number field.

Proof. For $n \geq 4$ this follows from local rigidity, see [BG04, Theorem 3.9], or [OV00, Proposition 1.6.5]. For $n=3$, see [MR03, Theorem 3.1.2].

3.1.3 Definition for manifolds with boundary. We want to extend this definition to manifolds with boundary. In order to do this, we will assume that our manifolds have finite volume and totally geodesic boundary components also of finite volume.

Lemma 3.7. Let $M$ be a manifold with boundary. Assume that the components of $\partial M$ are totally geodesic and that both $M$ and $\partial M$ have finite volume. Let $\widetilde{M} \subset \mathbf{H}^{n}$ be a universal cover for $M$ (which is isometrically embedded, as in Proposition 2.18) and let $\Gamma \subset \mathrm{PO}(n, 1)$ be the corresponding group of deck transformations. Then $\Gamma \cap \mathbf{P} \mathbf{O}_{(n, 1)}^{\circ}$ is Zariski-dense in $\mathbf{P O}_{(n, 1)}^{\circ}$.

Proof. Lemma 1.7B in [GPS87].

Therefore the trace field of $\Gamma$ can be defined as in Section 3.1.1, and similarly for the ambient group. Observe that since any finite-volume hyperbolic manifold with totally geodesic finite-volume boundary is the piece of a finitevolume hyperbolic manifold (namely its double, c.f. Section 2.2.2), its trace field is necessarily a number field. As in the previous section, we will call ambient group of $\Gamma$ any algebraic group $\mathbf{G}$ defined over its trace field $k$, such that $\mathbf{G}_{\mathbb{R}} \cong \mathbf{P O}_{(n, 1)}$ and $\Gamma \subset \mathbf{G}(k)$ via this isomorphism (see Remark 3.1.6).

\section{$3.2 \mid$ Manifolds with hypersurfaces}

This section is devoted to the study of the ambient group of a general hyperbolic manifold admitting a hypersurface. Our goal is to prove:

Theorem 3.8. Let $n \geq 3$ and $\Gamma \subset \mathrm{PO}(n, 1)$ be a torsion-free lattice such that the corresponding manifold $M=\Gamma \backslash \mathbf{H}^{n}$ contains an immersed hypersurface. Then the ambient group of $\Gamma$ is $\mathbf{P} \mathbf{O}_{f}$, where $f$ is some quadratic form defined over the trace field of $\Gamma$.

This is stated in [LM93], and follows also from [Me17, Proof of Theorem E], except for the case of trialitarian groups of type $D_{4}$ in dimension 7 (note however that the proof contains a small gap for $n \leq 7$ since the final rank 
inequality is not satisfied there). We will give a proof which also covers the case of groups of type $D_{4}$.

The main tool in proving Theorem 3.8 will be a proposition about reflections through hypersurfaces, which is interesting in its own right. This proposition and its proof are the subject of next section.

3.2.1 Rational reflections. The goal of this section is to prove the following proposition and use it to deduce Theorem 3.8.

Proposition 3.9 (Reflections are rational). Let $M$ be a hyperbolic manifold with trace field $k$ and ambient group $\mathbf{G}$, and identify $\mathbf{G}(\mathbb{R})$ with $\mathrm{PO}(n, 1)$. Let $R \subset \mathbf{H}^{n}$ be a hyperplane lift of an immersed hypersurface $N \subset M$, and let $\rho \in \mathbf{G}(\mathbb{R})$ denote the reflection through $R$. Then $\rho \in \mathbf{G}(k)$.

We will first need a lemma:

Lemma 3.10. Let $\mathbf{G}=\mathbf{O}_{n}(\mathbb{C})$, seen as an algebraic group over $\mathbb{C}$. Let $R \subset \mathbb{C}^{n}$ be a hyperplane with corresponding reflection $\rho \in \mathbf{G}$ and let $\mathbf{G}_{0} \subset \mathbf{G}$ be a Zariski-closed subgroup such that $\left\langle\mathbf{G}_{0}, \rho\right\rangle=\operatorname{Stab}_{\mathbf{G}}(R)$. Then the centralizer $\mathrm{Z}_{\mathbf{G}}\left(\mathbf{G}_{0}\right)$ of $\mathbf{G}_{0}$ in $\mathbf{G}$ equals $\{ \pm I, \pm \rho\}$, where $I$ is the identity matrix.

Proof. Up to conjugation, we may assume that $R=\left\{x_{n}=0\right\}$. Then

$$
\operatorname{Stab}_{\mathbf{G}}(R)=\mathbf{O}_{n-1}(\mathbb{C}) \times\{ \pm 1\}
$$

diagonally embedded in $\mathbf{G}$, and a straightforward computation shows that $\mathrm{Z}_{\mathbf{G}}\left(\operatorname{Stab}_{\mathbf{G}}(R)\right)=\{ \pm I, \pm \rho\}$. Thus it is enough to prove that the group $\mathrm{Z}_{\mathbf{G}}\left(\mathbf{G}_{0}\right)$ equals $\mathrm{Z}_{\mathbf{G}}\left(\operatorname{Stab}_{\mathbf{G}}(R)\right)$.

Let $g \in \mathbf{Z}_{\mathbf{G}}\left(\mathbf{G}_{0}\right)$. Since $\left\langle\mathbf{G}_{0}, \rho\right\rangle=\operatorname{Stab}_{\mathbf{G}}(R)$, it suffices to show that $g$ commutes with $\rho$. For a contradiction, assume not. This implies $g \rho g^{-1} \neq \rho$ and thus $g R \neq R$. But every $g_{0} \in \mathbf{G}_{0}$ commutes with $g \rho g^{-1}$, whence fixes $g R$. Therefore

$$
\mathbf{G}_{0} \subset \operatorname{Stab}_{\mathbf{G}}(R) \cap \operatorname{Stab}_{\mathbf{G}}(g R) \cong \mathbf{O}_{n-2}(\mathbb{C}) \times\{ \pm 1\} \times\{ \pm 1\} .
$$

This contradicts $\operatorname{dim} \mathbf{G}_{0}=\operatorname{dim} \mathbf{O}_{n-1}(\mathbb{C})$.

Proof of Proposition 3.9. We will identify $\mathbf{G}(\mathbb{R})$ and $\mathrm{PO}(n, 1)$ for convenience. Let $\Gamma_{0}=\operatorname{Stab}_{\Gamma}(R)$, and let $\mathbf{G}_{0}$ be the Zariski-closure of $\Gamma_{0}$ in $\mathbf{G}$.

We claim that the centralizer $\mathbf{Z}=\mathrm{Z}_{\mathbf{G}}\left(\mathbf{G}_{0}\right)$ of $\mathbf{G}_{0}$ in $\mathbf{G}$ consists of the two elements $\{1, \rho\}$. Since the hypersurface $N \cong \Gamma_{0} \backslash R$ has finite volume, Borel's density theorem implies $\left\langle\mathbf{G}_{0}, \rho\right\rangle=\operatorname{Stab}_{\mathbf{G}}(R)$. Now we can choose an isomorphism $\mathbf{G}(\mathbb{C}) \cong \mathbf{O}_{n+1}(\mathbb{C}) /\{ \pm 1\}$, lift our groups to $\mathbf{O}_{n+1}(\mathbb{C})$ and apply Lemma 3.10 to deduce the claim.

By Zariski-density,

$$
\mathbf{Z}=\bigcap_{\gamma \in \Gamma_{0}}\{g \in \mathbf{G} \mid g \gamma=\gamma g\}
$$


and $\mathbf{Z}$ is thus $k$-closed. Since it has two elements and $1 \in \mathbf{Z}(k)$ is fixed by all $k$-automorphisms of $\mathbb{C}$, the same must hold for $\rho$. Thus $\rho \in \mathbf{Z}(k) \subset \mathbf{G}(k)$.

We are ready for the:

Proof of Theorem 3.8. By definition, the ambient group of $\Gamma$ is a $k$-form of $\mathbf{P O}_{(n, 1)}$ (see Remark 3.1.6). If $n$ is even, there is thus nothing to do (the only possible $k$-forms of $\mathrm{PO}(n, 1)$ are $\mathbf{P O}_{f}$, see Theorem 2.8). Assume $n$ odd. Let $\mathbf{G}$ be the ambient group of $\Gamma, k$ its trace field. Identify $\mathbf{G}(\mathbb{R})$ with $\mathrm{PO}(n, 1)$. The embedded hypersurface lifts to a hyperplane $R \subset \mathbf{H}^{n}$, whose reflection $\rho$ is in $\mathbf{G}(k)$, by Proposition 3.9. The group $\operatorname{Stab}_{\mathbf{G}(\mathbb{R})}(R)$ is the real points of an algebraic subgroup $\mathbf{G}_{0} \subset \mathbf{G}_{\mathbb{R}}$ defined by

$$
\mathbf{G}_{0}=\mathbf{Z}_{\mathbf{G}}(\rho)=\{g \in \mathbf{G} \mid g \rho=\rho g\} .
$$

It is thus a $k$-subgroup of $\mathbf{G}$, and it follows (again by the classification of $k$-forms of $\left.\mathbf{P} \mathbf{O}_{(n-1,1)}\right)$ that $\mathbf{G}_{0}$ is isomorphic to $\mathbf{O}_{f_{0}}$, for some quadratic form $f_{0}$ defined over $k$.

Let $f=f_{0}+x_{n}^{2}$, where $x_{n}$ is a variable not occurring in $f_{0}$. We consider the model $\mathbf{H}_{f}$ for $\mathbf{H}^{n}$, with isometry group $\mathbf{P} \mathbf{O}_{f}(\mathbb{R})$. It is clear that there exists an isomorphism $\varphi: \mathbf{G}_{K} \rightarrow \mathbf{P O}_{f, K}$ defined over some finite field extension $K / k$, which we assume to be Galois. Furthermore, we can assume that the restriction $\left.\varphi\right|_{\mathbf{G}_{0, K}}$ is the $k$-isomorphism $\psi: \mathbf{G}_{0} \rightarrow \mathbf{O}_{f_{0}}$ from the previous paragraph, i.e., that $\varphi$ is such that

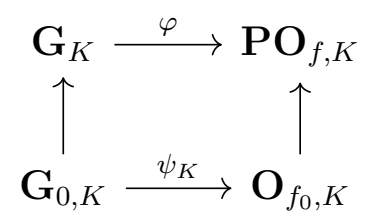

commutes.

Let $\sigma \in \operatorname{Gal}(K / k)$, and consider the automorphism of $\mathbf{P O}_{f, K}$ given by

$$
\alpha={ }^{\sigma} \varphi \circ \varphi^{-1}: \mathbf{P O}_{f, K} \stackrel{\varphi^{-1}}{\longrightarrow} \mathbf{G}_{K} \stackrel{{ }^{\sigma} \varphi}{\longrightarrow} \mathbf{P O}_{f, K}
$$

By the classification of automorphisms of $\mathbf{P O} \mathbf{O}_{f, K}$ (see Theorem 2.11), there is a $g \in \mathbf{P O}_{f}(K)$ such that

$$
\alpha(x)=g x g^{-1} \text {. }
$$

Moreover, since the isomorphism $\mathbf{G}_{0} \cong \mathbf{O}_{f_{0}}$ is over $k$, we have that

$$
\left.\alpha\right|_{\mathbf{O}_{f_{0}, K}}=\mathrm{id},
$$

and thus $g$ must commute with all elements of $\mathbf{O}_{f_{0}}$. Therefore (by Lemma 3.10) $g \in\{$ id, $\rho\}$, where $\rho \in \mathbf{P O}_{f}(k)$ is the reflection through the quadratic subspace defined by $\left\{x_{n}=0\right\}$. 
Let $\beta: \mathbf{P O} \mathbf{O}_{f} \rightarrow \mathbf{P O}$ denote the automorphism of $\mathbf{P O}$ induced via conjugation by $\rho$. It follows that for every $\sigma \in \operatorname{Gal}(K / k)$, we have

$$
{ }^{\sigma} \varphi \circ \varphi^{-1} \in\{\mathrm{id}, \beta\}
$$

Since id and $\beta$ are $k$-automorphisms, we have

$$
{ }^{\sigma \tau} \varphi \circ \varphi^{-1}={ }^{\sigma}\left({ }^{\tau} \varphi \circ \varphi^{-1}\right) \circ{ }^{\sigma} \varphi \circ \varphi^{-1}={ }^{\tau} \varphi \circ \varphi^{-1} \circ{ }^{\sigma} \varphi \circ \varphi^{-1}
$$

and it follows that ${ }^{\sigma^{2}} \varphi \circ \varphi^{-1}=\left({ }^{\sigma} \varphi \circ \varphi^{-1}\right)^{2}=\mathrm{id}$.

Let $N=\left\langle\sigma^{2} \mid \sigma \in \operatorname{Gal}(K / k)\right\rangle$; it is a normal subgroup of $\operatorname{Gal}(K / k)$, and thus the extension

$$
\ell=K^{N} / k
$$

is Galois. Since the square of all elements of the group $\operatorname{Gal}(\ell / k) \cong \operatorname{Gal}(K / k) / N$ is trivial, it is a group isomorphic to a product of $\mathbb{Z} / 2$. Thus $\ell$ is a multiquadratic extension of $k$, and since (by the previous paragraph)

$$
{ }^{\sigma} \varphi \circ \varphi^{-1}=\text { id } \quad \forall \sigma \in N
$$

we have that $\varphi$ is actually defined over $\ell$.

Write $\ell=k\left(\sqrt{a_{1}}, \ldots, \sqrt{a_{r}}\right)$, and let $\sigma_{i} \in \operatorname{Gal}(\ell / k)$ be the automorphism sending $\sqrt{a_{i}}$ to $-\sqrt{a_{i}}$ and fixing $\sqrt{a_{j}}$ for $j \neq i$. Let $\left\{i_{1}, \ldots, i_{s}\right\}$ be the set of indices such that ${ }^{\sigma_{i}} \varphi \circ \varphi^{-1}=\beta$, and define the quadratic form

$$
q=f_{0}+a x_{n}^{2}, \quad \text { where } a=a_{i_{1}} \cdots a_{i_{s}} .
$$

Then $q$ is a quadratic form over $k$, which becomes isomorphic to $f$ over $\ell$. More precisely, we have

$$
q=f \circ A, \quad \text { where } A=\left(\begin{array}{cc}
I & 0 \\
0 & \sqrt{a}
\end{array}\right) .
$$

Let $\psi: \mathbf{P O}_{q, \ell} \rightarrow \mathbf{P} \mathbf{O}_{f, \ell}$ be the corresponding isomorphism of algebraic groups.

By construction, ${ }^{\sigma_{i}} \psi \circ \psi^{-1}$ equals $\beta$ exactly when $i \in\left\{i_{1}, \ldots, i_{s}\right\}$, i.e., when ${ }^{\sigma_{i}} \varphi \circ \varphi^{-1}=\beta$. Hence

$$
{ }^{\sigma} \varphi \circ \varphi^{-1}={ }^{\sigma} \psi \circ \psi^{-1} \quad \forall \sigma \in \operatorname{Gal}(\ell / k)
$$

Consider the isomorphism $\eta: \mathbf{P O}_{q, \ell} \rightarrow \mathbf{G}_{\ell}$ given by $\eta=\varphi^{-1} \circ \psi$. Then the above equation rewrites as

$$
{ }^{\sigma} \varphi \varphi^{-1}={ }^{\sigma} \psi \psi^{-1} \Leftrightarrow{ }^{\sigma} \varphi \varphi^{-1} \psi={ }^{\sigma} \psi \Leftrightarrow \varphi^{-1} \psi={ }^{\sigma}\left(\varphi^{-1}\right)^{\sigma} \psi \Leftrightarrow \eta={ }^{\sigma} \eta
$$

for all $\sigma \in \operatorname{Gal}(\ell / k)$. Thus $\eta$ is defined over $k$ and $\mathbf{G} \cong \mathbf{P O}_{q}$, as desired. 
3.2.2 Manifolds of type I. It follows from the results of the previous section that any finite-volume hyperbolic manifold $M$ with non-empty finite-volume totally geodesic boundary satisfies the following:

1. The trace field $k$ of $M$ is a number field, naturally embedded in $\mathbb{R}$.

2. The ambient group of $M$ is $\mathbf{P O}_{f}$, where $f$ is a quadratic form over $k$ such that $\mathbf{P O}_{f}(\mathbb{R}) \cong \mathrm{PO}(n, 1)$.

This last property suggests that the natural setting to study these manifolds is by using the hyperbolic models $\mathbf{H}_{f}$ defined in Section 2.2.5.

Definition 3.2.1. A manifold of type $I$ is a hyperbolic manifold of finitevolume with (possibly empty) boundary, all of whose boundary components are totally geodesic and of finite volume, such that its ambient group is $\mathbf{P O} \mathbf{O}_{f}$ for some quadratic form $f$. If $M$ is a manifold of type I with trace field $k$, a model for $M$ is a universal cover $\widetilde{M} \subset \mathbf{H}_{f}$ (that is isometrically embedded, as in Proposition 2.18) with a covering map $\widetilde{M} \rightarrow M$ such that the corresponding group of deck transformations $\Gamma$ lies in $\mathbf{P O}_{f}(k)$.

To end this section, we give two results which we will need in the sequel. The first is about hypersurfaces in manifolds of type I, and is a corollary of Proposition 3.9.

Proposition 3.11. Let $M$ be a manifold of type I, and let $\widetilde{M} \subset \mathbf{H}_{f}$ be a model for $M$. Let $N \subset M$ be a hypersurface in $M$. Then any hyperplane lift $R \subset \mathbf{H}_{f}$ of $N$ is defined over $k$ (i.e., corresponds to the f-orthogonal complement of a vector $\left.x \in k^{n+1}\right)$.

Proof. Let $\mathbf{G O}_{f}$ denote the group of similitudes of $f$ (that is, the extension of the orthogonal group $\mathbf{O}_{f}$ by the multiplicative group $\mathbf{G}_{m}$, see [INV98, $§ 12$ and §23]). Then $\mathbf{P O}_{f}$ fits in the exact sequence of algebraic groups

$$
1 \rightarrow \mathbf{G}_{m} \rightarrow \mathbf{G O}_{f} \rightarrow \mathbf{P O}_{f} \rightarrow 1,
$$

and since $H^{1}\left(k, \mathbf{G}_{m}\right)=0$ (Hilbert's Theorem 90) we have that the induced map $\mathbf{G O}_{f}(k) \rightarrow \mathbf{P O}_{f}(k)$ is surjective (see [Se68, Proposition 1, p. 133]).

By Proposition 3.9, the reflection $\rho$ at $R$ lifts to an element $\widetilde{\rho} \in \mathbf{G O}_{f}(k)$. Let $V$ denote the $k$-quadratic space defined by $f$, and let $W \subset V \otimes_{k} \mathbb{R}$ be the subspace corresponding to $R$. Since $W$ is described by the equation

$$
W=\left\{x \in V \otimes_{k} \mathbb{R} \mid \widetilde{\rho} x=x\right\},
$$

it is defined over $k$. Thus so is its orthogonal complement in $V$, i.e., there is $v \in V$ such that $W=v^{\perp_{f}} \otimes_{k} \mathbb{R}$, as desired.

The final lemma is a technical result which explains how isometries of manifolds of type I induce isometries of the models. 
Lemma 3.12. Let $M_{1}, M_{2}$ be manifolds of type $I$, and for $i=1,2$ let $\widetilde{M}_{i} \subset \mathbf{H}_{f_{i}}$ be a model for $M_{i}$. Assume there exists an isometry $\phi: M_{1} \rightarrow M_{2}$, and let $k$ denote the trace field of $M_{1}$ (and thus of $M_{2}$ ). Then $\phi$ lifts to an isometry $\mathbf{H}_{f_{1}} \rightarrow \mathbf{H}_{f_{2}}$ induced by a matrix $A \in \mathrm{GL}_{n+1}(k)$ such that $f_{2} \circ A=\lambda f_{1}$ for some $\lambda \in k$.

Proof. The isometry $\phi$ induces an isomorphism $\Phi: \mathbf{P O}_{f_{1}} \rightarrow \mathbf{P O}_{f_{2}}$ of the ambient groups which is defined over $k$. By Theorem 2.14, this isomorphism is induced by a matrix $A \in \mathrm{GL}_{n+1}(k)$ such that $f_{2} \circ A=\lambda f_{1}$ for some $\lambda \in k$. In turn, the isometry $\mathbf{H}_{f_{1}} \rightarrow \mathbf{H}_{f_{2}}$ induced by $A$ coincides with $\phi$ (since they induce the same isomorphism $\mathbf{P O} \mathbf{f}_{f_{1}} \rightarrow \mathbf{P O}{\mathbf{f}_{2}}_{2}$.

\subsection{Computation in the arithmetic case}

This section is devoted to the computation of the trace field and ambient group of arithmetic groups. Let $\Gamma \subset \mathrm{PO}(n, 1)$ be an arithmetic lattice. By definition, there exists an admissible algebraic group $\mathbf{G}$ defined over a totally real number field $k \subset \mathbb{R}$ such that $\mathbf{G}(\mathbb{R}) \cong \mathrm{PO}(n, 1)$ and $\Gamma$ is commensurable with the image of $\mathbf{G}\left(\mathcal{O}_{k}\right)$ in $\mathrm{PO}(n, 1)$.

Theorem 3.13. Let $\Gamma$ be as above. Then the trace field of $\Gamma$ is $k$ and its ambient group is $\mathbf{G}$.

Proof. This follows from [PR09, Lemma 2.6], yet we will give an easier proof in our more specific case.

Identify $\mathbf{G}(\mathbb{R})$ with $\operatorname{PO}(n, 1)$. Since the trace field and ambient group are invariant under commensurability, we can assume that $\Gamma$ is in $\mathbf{G}(k)$. Thus it is sufficient to show that the trace field of $\Gamma$ coincides with $k$, since then it will follow from uniqueness of ambient groups that $\mathbf{G}$ is the ambient group of $\Gamma$.

Let $\ell$ be the trace field of $\Gamma$. Since the isomorphism $\mathbf{G}(\mathbb{R}) \cong \operatorname{PO}(n, 1)$ induces an isomorphism of the Lie algebras, the traces of the adjoint representations coincide and we have $\ell \subset k$. For the reverse inclusion we know from the previous section that there exists an algebraic group $\mathbf{F}$ defined over $\ell$ such that $\mathbf{F}(\mathbb{R}) \cong \mathrm{PO}(n, 1)$ and $\Gamma \subset \mathbf{F}(\ell)$ via this isomorphism. By Proposition 2.13, we see that $\mathbf{G} \cong \mathbf{F}_{k}$. Since $k$ is totally real, each embedding $\ell \hookrightarrow \mathbb{R}$ extends to exactly $[k: \ell]$ embeddings $k \hookrightarrow \mathbb{R}$. Moreover, as

$$
\mathbf{G}\left(k \otimes_{\mathbb{Q}} \mathbb{R}\right)=\mathbf{F}\left(k \otimes_{\mathbb{Q}} \mathbb{R}\right) \cong \mathbf{F}\left(\ell \otimes_{\mathbb{Q}} \mathbb{R}\right)^{[k: \ell]}
$$

the number of non-compact factors in $\mathbf{G}\left(k \otimes_{\mathbb{Q}} \mathbb{R}\right)$ is a multiple of $[k: \ell]$. Since $\mathbf{G}$ is admissible, there is only one such factor. This forces $[k: \ell]=1$, and the theorem is proven. 
Finally, we give a corollary about the trace field of arithmetic manifolds sharing a hypersurface.

Corollary 3.14. Let $M_{1}, M_{2}$ be arithmetic manifolds of type I, with hypersurfaces $N_{i} \subset M_{i}$. Assume $N_{1}$ and $N_{2}$ are commensurable. Then the trace fields of $M_{1}$ and $M_{2}$ coincide.

Proof. By Theorem 2.25, the hypersurfaces $N_{i}$ are arithmetic and commensurable to a common manifold $N_{0}=\Gamma_{0} \backslash \mathbf{H}_{f_{0}}$ with $\Gamma_{0}$ commensurable to $\mathbf{P O}_{f_{0}}\left(\mathcal{O}_{k}\right)$, for some admissible quadratic form $f_{0}$. Thus their trace field is $k$, and again by Theorem 2.25, this is the trace field of $M_{1}$ and $M_{2}$. 


\section{Chapter $4 \mid$ Gluings}

This chapter introduces gluing constructions and gives tools to compute their trace fields and ambient groups. In the first section, we start by defining gluings in a broad sense that encompasses all known constructions. We then review in Section 4.2 the classical gluing constructions of nonarithmetic manifolds using pieces of arithmetic manifolds (the method of Gromov and Piatetski-Shapiro and its generalizations).

In Section 4.3 we proceed to state and prove the main results of this thesis (concerning gluings). The idea is that an isometry used to glue pieces of manifolds together at a given hypersurface admits naturally a field of definition, which turns out to coincide with the trace field of the resulting gluing. This allows one to compute the trace field of gluings easily by choosing appropriate models for the pieces. We apply this technique in Section 4.4 to deduce general corollaries of the main theorem and compute the trace field of manifolds obtained using the previously described classical gluing constructions.

In Section 4.5, we use these constructions with pieces of commensurable arithmetic manifolds and show that one can realize an arbitrary large trace field. Finally, we give examples (in any even dimension $n \geq 4$ ) of pieces of arithmetic manifolds whose trace field increases under "closing up" (see Definition 4.1.2).

\section{$4.1 \mid$ General hyperbolic gluings}

4.1.1 Interbreeding and closing up. In this section we introduce the notion of gluing of hyperbolic manifolds in its most general form. Since all our manifolds will have hypersurfaces (or boundary hypersurfaces) we will restrict our attention on manifolds of type I (see Section 3.2.2). Recall that those are finitevolume hyperbolic manifolds with (possibly empty) boundary, all of whose components are totally geodesic and of finite volume. Moreover, these are required to have an ambient group of the form $\mathbf{P O} \mathbf{O}_{f}$ (if they have (boundary) hypersurfaces, this is always satisfied, see Theorem 3.8).

Let $M_{1}$ and $M_{2}$ be manifolds of type I, and assume there are boundary hypersurfaces $N_{1} \subset \partial M_{1}$ and $N_{2} \subset \partial M_{2}$ which are isometric. Topologically, 
we can glue $M_{1}$ with $M_{2}$ together along these boundary components. More precisely, let $\varphi: N_{1} \rightarrow N_{2}$ be an isometry, and let $M$ denote the adjunction space

$$
M=M_{1} \cup_{\varphi} M_{2}=M_{1} \sqcup M_{2} / \sim_{\varphi}
$$

where $\sim_{\varphi}$ is the equivalence relation induced by $x \sim \varphi(x)$ for all $x \in N_{1}$. This is a topological space, which is naturally endowed with a structure of hyperbolic manifold with boundary, as we will see below.

Definition 4.1.1. The manifold $M=M_{1} \cup_{\varphi} M_{2}$ is called an interbreeding of $M_{1}$ and $M_{2}$ along $\varphi$.

This construction can also be applied to glue a manifold $M_{1}$ to itself. Indeed, let $M_{1}$ be a manifold of type I and let $N_{1}, N_{2} \subset \partial M_{1}$ be two disjoint isometric boundary hypersurfaces. Then if $\varphi: N_{1} \rightarrow N_{2}$ is an isometry, we can form the quotient space

$$
M=M_{1} / \sim_{\varphi}
$$

where $\sim_{\varphi}$ is the equivalence relation induced by $x \sim \varphi(x)$ for all $x \in N_{1}$. As before, this space admits a natural structure of hyperbolic manifold with boundary, as we will see in the next theorem.

Definition 4.1.2. The manifold $M=M_{1} / \sim_{\varphi}$ is called the closing up of $M_{1}$ along $\varphi$.

The following theorem justifies the fact that we call these constructions manifolds.

Theorem 4.1. Interbreedings and closing ups have a natural complete structure of hyperbolic manifold around the glued hypersurface $N_{1}=N_{2} \subset M$.

Proof. The proof is the same as that of Theorem 2.10.B in [GPS87], which treats the special case in which $M_{1}$ and $M_{2}$ have only one or two boundary components. It is to be noted that the condition that $N_{1}$ and $N_{2}$ have finite volume is essential.

The common object that will play a central role in trace field computations is the following.

Definition 4.1.3. The isometry $\varphi: N_{1} \rightarrow N_{2}$ used in the construction of interbreedings and closing ups is called the gluing isometry.

In general, we want to look at gluings which have pre-defined pieces as building blocks, whence the next definition.

Definition 4.1.4. Let $\mathcal{M}$ be a set of hyperbolic $n$-manifolds of type I, of fixed dimension $n$. Then the gluings of $\mathcal{M}$ is the smallest set $\mathcal{M}^{\text {gl }}$ containing $\mathcal{M}$ and closed under interbreedings and closing ups.

Thus for each manifold $M \in \mathcal{M}^{g l}$ there exists a sequence $M_{0}, \ldots, M_{r}$ with $M_{0} \in \mathcal{M}, M_{r}=M$ and $M_{i+1}$ obtained either by closing up $M_{i}$ or interbreeding it with a manifold in $\mathcal{M}$. 
4.1.2 Arithmetic pieces. The main examples of gluings for this thesis will be constructed using arithmetic pieces:

Example 4.1.5. Let $\mathcal{A}$ denote the set of all pieces of arithmetic $n$-manifolds. Then the elements of the set $\mathcal{A}^{g l}$ are gluings of arithmetic pieces. As said in the introduction, as of today, the known examples of nonarithmetic manifolds arise either from hyperbolic Coxeter groups or from gluing constructions involving arithmetic pieces. Since finite covolume (resp. cocompact) Coxeter groups do not exist in dimensions $n \geq 996$ (resp. $n \geq 30$ ), see [Pr86, Vi81], we see that the set $\mathcal{A}^{g l}$ contains all known examples of nonarithmetic hyperbolic manifolds large dimension.

It was however recently shown by Fisher, Lafont, Miller and Stover [FLMS18] that there exists a hyperbolic manifold $M$ of dimension 5 which is not commensurable to a gluing of non-commensurable arithmetic pieces. Therefore $M \notin \mathcal{A}^{g l}$. However, it turns out that this manifold $M$ is still pseudo-arithmetic (see [EM18] and Chapter 5).

In Section 2.3.3 we explained how to construct arithmetic hyperbolic manifolds containing hypersurfaces. We will now use this process to produce arithmetic pieces with prescribed boundary (up to commensurability).

Let $k \subset \mathbb{R}$ be a totally real number field, and let $f=f_{0}+a x_{n}^{2}$ be an admissible quadratic form over $k$, with $f_{0}=f_{0}\left(x_{0}, \ldots, x_{n-1}\right)$ and $a \in k$ totally positive. Let $\Gamma \subset \mathbf{P O}_{f}(k)$ be the image of $\mathbf{O}\left(\mathcal{O}_{k}\right)$ in $\mathbf{P} \mathbf{O}_{f}$. It is an arithmetic lattice in $\mathbf{P O}_{f}(\mathbb{R})$ and it follows from Selberg's lemma (Theorem 2.19) that there exists a congruence subgroup $\Gamma_{1} \subset \Gamma$ which is torsion-free, i.e., such that $\Gamma_{1} \backslash \mathbf{H}_{f}$ is a hyperbolic manifold.

Let $R \subset \mathbf{H}_{f}$ be the hyperplane defined by the subspace $\left\{x_{n}=0\right\}$. As seen in Theorem 2.25, the subgroup $\Lambda=\operatorname{Stab}_{\Gamma}(R)$ is a lattice in the group $\operatorname{Stab}_{\mathbf{P O}_{f}(\mathbb{R})}(R) \cong \mathbf{O}_{f_{0}}(\mathbb{R})$. Therefore, by subgroup separability (Theorem 2.21) there exists a further finite-index subgroup $\Gamma_{2} \subset \Gamma_{1}$ such that the map $\Lambda_{2} \backslash R \hookrightarrow \Gamma_{2} \backslash \mathbf{H}_{f}$ is an embedding, where $\Lambda_{2}=\operatorname{Stab}_{\Gamma_{2}}(R)$. In fact, since we are dealing with arithmetic manifolds, we can use Theorem 2.26 with one hyperplane, which allows us to take $\Gamma_{2}$ as a congruence subgroup.

The hyperplane $R$ maps onto a hypersurface in $M$. The next proposition shows that by taking a further finite index subgroup, we can ensure that the collection of hyperplanes $\Gamma_{2} \cdot R$ projects down to as many copies of $N$ as we want:

Proposition 4.2. Let $M$ be an orientable arithmetic manifold and $N \subset M$ an orientable embedded hypersurface. Then for any $m \in \mathbb{N}$, there exists a finite normal covering $M^{\prime} \rightarrow M$ such that there are $m$ disjoint lifts $N_{1}, \ldots, N_{m} \subset M^{\prime}$ of $N$, all isometric to it, such that $M^{\prime} \backslash \bigcup N_{i}$ is connected.

Proof. Proposition 4.3 of [GL14]. 
It follows that for each $m$, there exists a (normal) finite-index subgroup $\Gamma_{3} \subset \Gamma_{2}$ such that $M_{3}=\Gamma_{3} \backslash \mathbf{H}_{f}$ contains $m$ disjoint copies $N_{1}, \ldots N_{m}$ of $N$, and such that $M_{3} \backslash \bigcup N_{i}$ is connected. Thus its completion $M$ is a piece of arithmetic manifold which has $2 m$ boundary components, all of which are isometric to $N$.

We sum this up in a proposition-definition that we will use later.

Proposition 4.3. Let $k \subset \mathbb{R}$ be a totally real number field, $f=f_{0}+a x_{n}^{2} a$ quadratic form over $k$, with $f_{0}=f_{0}\left(x_{0}, \ldots, x_{n-1}\right)$ admissible and $a \in k$ totally positive. Let $\Gamma \subset \mathbf{P} \mathbf{O}_{f}(k)$ denote the image of the group $\mathbf{O}_{f}\left(\mathcal{O}_{k}\right)$ in $\mathbf{P} \mathbf{O}_{f}$, and for an ideal $\mathfrak{a} \subset \mathcal{O}_{k}$ let $\Gamma(\mathfrak{a}) \subset \Gamma$ denote the congruence subgroup of $\Gamma$ of level $\mathfrak{a}$, i.e., the image of the group $\operatorname{ker}\left(\mathbf{O}_{f}\left(\mathcal{O}_{k}\right) \rightarrow \mathbf{O}_{f}\left(\mathcal{O}_{k} / \mathfrak{a}\right)\right)$ in $\mathbf{P O}_{f}$. Let $R \subset \mathbf{H}_{f}$ denote the hyperplane corresponding to the subspace $\left\{x_{n}=0\right\}$, let $\Lambda=\operatorname{Stab}_{\Gamma}(R)$ and set $\Lambda(\mathfrak{a})=\Lambda \cap \Gamma(\mathfrak{a})$. Then:

1. $\Lambda(\mathfrak{a})$ is the image of $\operatorname{ker}\left(\mathbf{O}_{f_{0}}\left(\mathcal{O}_{k}\right) \rightarrow \mathbf{O}_{f_{0}}\left(\mathcal{O}_{k} / \mathfrak{a}\right)\right)$ in $\mathbf{P O}_{f}$.

2. There exists an ideal $\mathfrak{a} \subset \mathcal{O}_{k}$ such that $\Gamma(\mathfrak{a})$ is torsion-free and the covering $\mathbf{H}_{f} \rightarrow M=\Gamma(\mathfrak{a}) \backslash \mathbf{H}_{f}$ induces an embedding

$$
N=\Lambda(\mathfrak{a}) \backslash \mathbf{H}_{f_{0}} \cong \Lambda(\mathfrak{a}) \backslash R \hookrightarrow M=\Gamma(\mathfrak{a}) \backslash \mathbf{H}_{f}
$$

with both $N$ and $M$ orientable.

3. For such an $\mathfrak{a}$ and each $m \in \mathbb{N}$, there exists a finite-index normal subgroup $\Gamma^{\prime} \subset \Gamma(\mathfrak{a})$ and $m$ elements $\gamma_{1}, \ldots, \gamma_{m} \in \Gamma(\mathfrak{a})$ such that the hyperplanes $\gamma_{i} R$ project to disjoint hypersurfaces $N_{i} \subset M^{\prime}=\Gamma^{\prime} \backslash \mathbf{H}_{f}$, all isometric to $N$ and such that $M^{\prime} \backslash \bigcup N_{i}$ is connected.

Definition 4.1.6. An arithmetic piece of type $\left(f=f_{0}+a x_{n}^{2}, \mathfrak{a} \subset \mathcal{O}_{k}\right)$ is a piece obtained as the completion of a connected component of $M \backslash N$, where $M, N, f, \mathfrak{a}$ are as in 2. of Proposition 4.3. An arithmetic piece of type $(f, \mathfrak{a}, m)$ is a piece obtained as the completion of $M^{\prime} \backslash \bigcup N_{i}$, where $M^{\prime}, N_{i}, f, \mathfrak{a}, m$ are as in 3. of Proposition 4.3.

We end this section by introducing for each boundary hypersurface a canonical choice of model for arithmetic pieces. Let $M$ be an arithmetic piece of type $(f, \mathfrak{a})$ or $(f, \mathfrak{a}, m)$ and let $N \subset \partial M$ be a hypersurface in the boundary. By definition, $M$ is a piece of $\Gamma_{1} \backslash \mathbf{H}_{f}$, where $\Gamma_{1}$ is a finite-index subgroup of the congruence subgroup $\Gamma(\mathfrak{a})$ and $\Gamma$ is the image of $\mathbf{O}_{f}\left(\mathcal{O}_{k}\right)$ in $\mathbf{P O}_{f}$. Let $\widetilde{M} \subset \mathbf{H}_{f}$ be a model for $M$ with monodromy representation $\Gamma^{+} \subset \Gamma_{1} \subset \mathbf{P O}_{f}(k)$ such that $\Gamma^{+}=\operatorname{Stab}_{\Gamma}(\widetilde{M})$, and let $R \subset \partial \widetilde{M}$ be a hyperplane lift of $N$. Then there exists an element $\gamma \in \Gamma(\mathfrak{a})$ such that $\gamma R$ is the hyperplane $R_{0}$ corresponding to $\left\{x_{n}=0\right\}$. Moreover, it is easy to see that such a $\gamma$ is unique up to multiplying with elements of $\operatorname{Stab}_{\Gamma^{+}}\left(R_{0}\right)$ on the left. 
Let $\pi: \widetilde{M} \rightarrow M$ denote the covering map, and let $\widetilde{M}_{N}=\gamma \widetilde{M}$. Then the composition $\pi \circ \gamma^{-1}: \widetilde{M}_{N} \rightarrow \widetilde{M} \rightarrow M$ is a universal covering of $M$, independent of the choice of $\gamma$. Moreover, since $\gamma \in \Gamma(\mathfrak{a}) \subset \mathbf{P O}_{f}(k)$, we see that $\widetilde{M}_{N} \subset \mathbf{H}_{f}$ is actually a model for $M$, with monodromy representation

$$
\gamma \Gamma^{+} \gamma^{-1} \subset \mathbf{P O}_{f}(k) \text {. }
$$

Definition 4.1.7. The model $\widetilde{M}_{N} \subset \mathbf{H}_{f}$ is called a canonical model for $M$ with respect to $N$.

This model will be useful when computing trace fields of gluings of arithmetic pieces.

\section{$4.2 \mid$ Nonarithmetic gluings}

This section surveys the existing gluing constructions for hyperbolic manifolds. These were originally introduced by Gromov and Piatetski-Shapiro as a way of obtaining nonarithmetic lattices in arbitrary dimension. We start by recalling their classical construction and then explore further generalizations.

4.2.1 Gromov and Piatetski-Shapiro's construction. We will consider the gluing construction of Gromov and Piatetski-Shapiro in the broadest sense. For $i=1,2$, let $M_{i}$ be an arithmetic piece of type $\left(f_{i}=f_{0}+a_{i} x_{n}^{2}, \mathfrak{a} \subset \mathcal{O}_{k}\right)$ (see Definition 4.1.6). Recall that this means that $f_{i}$ is an admissible quadratic form over a totally real number field $k \subset \mathbb{R}$, with (totally) positive $x_{n}^{2}$ coefficient, and that $\mathfrak{a} \subset \mathcal{O}_{k}$ is an ideal chosen such that the corresponding lattice $\Gamma(\mathfrak{a}) \subset \mathbf{P O}_{f_{i}}$ is torsion free and the hyperplane $R \subset \mathbf{H}_{f_{i}}$ corresponding to $\left\{x_{n}=0\right\}$ projects down to a hypersurface in the boundary $\partial M_{i}$ of $M_{i}$.

By construction, all boundary component(s) $M_{1}$ and $M_{2}$ are isometric to an arithmetic manifold $N$ corresponding to the quadratic form $f_{0}$, i.e.,

$$
N=\Lambda(\mathfrak{a}) \backslash \mathbf{H}_{f_{0}} \quad \text { where } \Lambda=\text { image of } \mathbf{O}_{f_{0}}\left(\mathcal{O}_{k}\right) \text { in } \mathbf{P} \mathbf{O}_{f_{0}} .
$$

Now assume further that the arithmetic manifolds corresponding to $f_{i}$ are non-commensurable (i.e., that the quadratic forms are not similar, see Theorem 2.24). Since $M_{1}$ and $M_{2}$ have isometric boundary component(s), we can glue them together, first interbreeding at one boundary component and closing up the (potentially) remaining boundary component; call $M$ the corresponding manifold. Observe that the gluing isometry is not specified. However this is not needed for the purpose of nonarithmeticity since we started with non-commensurable arithmetic groups:

Proposition 4.4. The gluing $M$ is nonarithmetic.

Proof. See [GPS87, §2.9].

There is however a canonical choice of gluing isometry, which we now define. 
Definition 4.2.1. For $i=1,2$, let $M_{i}$ be an arithmetic piece of type $\left(f_{i}, \mathfrak{a}\right)$ or $\left(f, \mathfrak{a}, m_{i}\right)$, for $f=f_{0}+a_{i} x_{n}^{2}, \mathfrak{a} \subset \mathcal{O}_{k}$ and $m_{i} \geq 1$. Let $N_{i} \subset \partial M_{i}$ be a hypersurface in the boundary, and let $\widetilde{M}_{i} \subset \mathbf{H}_{f_{i}}$ be the canonical model for $M_{i}$ with respect to $N_{i}$, such that the hyperplane $R_{i} \subset \mathbf{H}_{f_{i}}$ corresponding to $\left\{x_{n}=0\right\}$ is a hyperplane lift of $N_{i}$. Then the canonical gluing isometry $\varphi: N_{1} \rightarrow N_{2}$ is the map induced by the identification $R_{1}=\mathbf{H}_{f_{0}}=R_{2}$ in the universal covers of $N_{1}, N_{2}$.

We will see in further detail how the gluing isometry influences the trace field of the glued manifold (even when the pieces come from commensurable manifolds) in Sections 4.4 and 4.5.

4.2.2 Generalizations. The gluing construction of previous section can be generalized to construct many non-commensurable nonarithmetic manifolds, and give lower bounds on the number of commensurability classes of nonarithmetic manifolds of volume less than $v$, as $v \rightarrow \infty$. The idea is to glue many pieces together according to a specific colored graph, and to show that the resulting gluings are commensurable exactly when the graphs are isomorphic. This process was first used by Raimbault [Ra13] and generalized by Gelander and Levit [GL14]. We will briefly explain this latter construction.

Let $f_{0}\left(x_{0}, \ldots, x_{n-1}\right)$ be an admissible quadratic form over a totally real number field $k$, and for $i=1, \ldots, 6$, let $f_{i}=f_{0}+a_{i} x_{n}^{2}$ for some totally positive $a_{i} \in k$. Assume that the $f_{i}$ are pairwise non-similar (so that the arithmetic groups they define are pairwise non-commensurable) and let $M_{i}$ be an arithmetic piece of type $\left(f_{i}, \mathfrak{a} \subset \mathcal{O}_{k}, m_{i}\right)$, where $\mathfrak{a} \subset \mathcal{O}_{k}$ is a fixed ideal and

$$
m_{1}=m_{2}=2, \quad m_{3}=\cdots=m_{6}=1 .
$$

Since the pieces $M_{i}$ all have isometric boundary components, one can interbreed them together or close them up in any way we like (for example using the canonical gluing isometry of Definition 4.2.1). The idea of Gelander and Levit is to glue them along a graph $\Delta$ obtained as 2-coloring the quotient of the Cayley Graph of the free group $F$ on two generators $a$ and $b$ by a finite index subgroup $H \subset F$. Thus $\Delta$ is a 2-colored 4-regular oriented graph with edges labeled in $\left\{a, a^{-1}, b, b^{-1}\right\}$. Let $V_{1}=M_{1}$ and $V_{2}=M_{2}$, and define $A$ (resp. $A^{-1}$ ) to be the "oriented interbreeding" of $M_{3}$ with $M_{4}$ (resp. of $M_{4}$ with $M_{3}$ ). Let $B, B^{-1}$ be defined analogously with $M_{5}$ and $M_{6}$. Then to each graph $\Delta$ constructed above corresponds a glued manifold $M_{\Delta}$ constructed by taking a copy of $V_{1}$ or $V_{2}$ for each vertex (depending on the coloring) and by gluing the manifolds $A, A^{-1}, B, B^{-1}$ depending on the edges of $\Delta$. Again, the gluing isometries do not matter if one is interested in nonarithmeticity and non-commensurability:

Proposition 4.5. Let $\Delta, \Delta^{\prime}$ be two non-isomorphic graphs as above, each having a single vertex of color 1 . Then the two manifolds $M_{\Delta}$ and $M_{\Delta^{\prime}}$ are noncommensurable. 
Proof. See [GL14, Proposition 3.3].

By estimating the number of such colored graphs $\Delta$, they are able to construct many pairwise non-commensurable non-arithmetic manifolds.

Another important construction of nonarithmetic manifolds is due to Belolipetsky and Thomson [BT11] (following an idea of Agol [Ag06] in dimension 4). The idea is to construct, for each $\epsilon>0$, an arithmetic piece having two boundary components which are at distance $\leq \epsilon$ of each other (using Theorem 2.26). The double of such a piece then has systole $\leq 2 \epsilon$, and as $\epsilon \rightarrow 0$ such a manifold cannot be arithmetic.

It turns out that the manifolds of Agol-Belolipetsky-Thomson are all quasiarithmetic (see Thomson [Th16] for a proof, and Section 5.1 for a definition). It follows that the trace field of the gluing is the same as that of the original manifold. Alternatively, this is also a consequence of Proposition 4.11 below, which states that the trace field never increases when taking the double of a piece of a manifold (arithmetic or not). There is however a finer invariant called the trace ring which allows to distinguish between the commensurability classes of these manifolds, see [Mi18].

\subsection{Trace fields and ambient groups of gluings}

This section contains the main result of this thesis concerning gluings (Theorem 4.7). In the first part, we define extensions of gluing isometries, and show that they admit naturally a field of definition which depends only on the original gluing isometry. In the second part, we prove that it coincides with the trace field of the resulting gluing (interbreeding or closing up). This method will allow us to easily compute the trace field of gluings of manifolds of type I and derive interesting corollaries.

We will generally assume that the manifolds used in the gluing constructions are of dimension $n \geq 4$. The reason for this choice is that the trace field of hyperbolic surfaces and 3-manifolds is usually studied using the algebraic groups $\mathbf{P S L}_{2}(\mathbb{R})$ and $\mathbf{P S L}(\mathbb{C})$ instead of $\mathbf{P} \mathbf{O}_{(n, 1)}(\mathbb{R})$ for the isometries of $\mathbf{H}^{n}$, see Remark 3.1.4 and [MR03, Chapters 1 and 3]. For (invariant) trace field computations of gluings in dimension 3, see Neumann and Reid [NR91].

4.3.1 Field of definition of gluing isometries. In this section we will consider gluing isometries (see Definition 4.1.3), and attach a certain field to them. Let $M_{1}, M_{2}$ be manifolds of type I, and assume they have isometric boundary hypersurfaces $N_{1} \cong N_{2}$, where $N_{i} \subset \partial M_{i}$. Let $\varphi: N_{1} \rightarrow N_{2}$ be a gluing isometry (here we allow the closing up case by setting $M_{1}=M_{2}$ ). Let $\widetilde{M}_{i} \subset \mathbf{H}_{f}$ be a model for $M_{i}$ and let $R_{i} \subset \partial \widetilde{M}_{i}$ be a hyperplane lift of $N_{i}$. 
Definition 4.3.1. An extension of $\varphi$ is an isometry $\phi: \mathbf{H}_{f_{1}} \rightarrow \mathbf{H}_{f_{2}}$ sending $R_{1}$ to $R_{2}$ such that the following diagram commutes (where the vertical maps are the coverings):

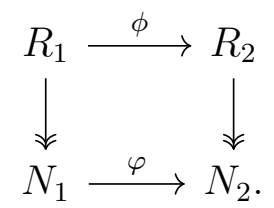

In terms of the monodromy representations $\Gamma_{i} \subset \mathbf{P O}_{f_{i}}\left(k_{i}\right)$ this implies that

$$
\phi \operatorname{Stab}_{\Gamma_{1}}\left(R_{1}\right) \phi^{-1}=\operatorname{Stab}_{\Gamma_{2}}\left(R_{2}\right) .
$$

Such a $\phi$ always exists. Indeed, the composition $R_{1} \rightarrow N_{1} \rightarrow N_{2}$ is a covering of $N_{2}$, and since $R_{1}$ is connected and simply connected, it is a universal covering of $N_{2}$. Thus $R_{1} \rightarrow N_{2}$ factors through $R_{2}$, and we get a map $\phi: R_{1} \rightarrow R_{2}$ which fits in a commutative diagram as above. Now it simply remains to arbitrarily extend $\phi$ to a map $\mathbf{H}_{f_{1}} \rightarrow \mathbf{H}_{f_{2}}$.

The isometry $\phi$ is induced via multiplication by a matrix $A \in \mathrm{GL}_{n+1}(\mathbb{R})$ such that $f_{1}=f_{2} \circ A$, and thus naturally induces (via conjugation) an isomorphism of algebraic groups

$$
\begin{aligned}
\Phi: \mathbf{P O}_{f_{1}, \mathbb{R}} & \longrightarrow \mathbf{P O}_{f_{2}, \mathbb{R}} \\
g & \longmapsto \phi g \phi^{-1} .
\end{aligned}
$$

This isomorphism is algebraic and hence admits a field of definition (see Section 2.1.2). The main lemma of this section is the following.

Lemma 4.6. The field of definition of $\Phi$ depends only on $\varphi$.

This motivates the following definition:

Definition 4.3.2. Let $\varphi$ be a gluing isometry. The field of definition of $\varphi$ is the field of definition of the induced isomorphism $\Phi$.

Proof of Lemma 4.6. We treat both the interbreeding and closing up case at once by setting $M_{1}=M_{2}$ in the latter. Let $\varphi: N_{1} \subset \partial M_{1} \rightarrow N_{2} \subset \partial M_{2}$ be a gluing isometry, let $\phi, \phi^{\prime}$ be two extensions and let $\Phi, \Phi^{\prime}$ be the corresponding isomorphisms on the ambient groups. By definition, $\phi$ and $\phi^{\prime}$ can be defined using arbitrary models for $M_{1}$ and $M_{2}$. The first step is to reduce the proof to the case where both $\phi$ and $\phi^{\prime}$ are defined using fixed models $\widetilde{M}_{1}$ for $M_{1}$ and $\widetilde{M}_{2}$ for $M_{2}$.

Let $\widetilde{M} \subset \mathbf{H}_{f}$ and $\widetilde{M}^{\prime} \subset \mathbf{H}_{f^{\prime}}$ be two models for $M_{1}$. By uniqueness of universal covers, there exists an isometry $\psi: \widetilde{M} \rightarrow \widetilde{M^{\prime}}$ such that the following diagram commutes, where the diagonal arrows are the covering maps:

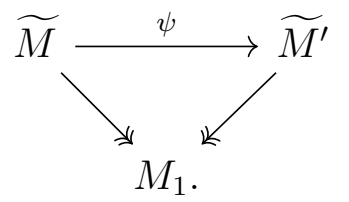


This isometry extends to an isometry $\psi: \mathbf{H}_{f} \rightarrow \mathbf{H}_{f^{\prime}}$, and if $\Gamma \subset \mathbf{P O}_{f}(k)$ and $\Gamma^{\prime} \subset \mathbf{P O}_{f^{\prime}}(k)$ are the monodromy representations corresponding to the above coverings, the commutative diagram implies that

$$
\psi \Gamma \psi^{-1}=\Gamma^{\prime}
$$

Since $\Gamma$ is (almost) Zariski-dense in $\mathbf{P O}_{f}$ and $\Gamma^{\prime} \subset \mathbf{P O} \mathbf{O}_{f}(k)$, it follows from Proposition 2.13 that the isomorphism $\Psi_{1}: \mathbf{P O}_{f, \mathbb{R}} \rightarrow \mathbf{P O}_{f^{\prime}, \mathbb{R}}$ induced by $\psi$ is defined over $k_{1}$, the trace field of $M_{1}$. Similarly, for $M_{2}$ any two choices of models give rise to such an isomorphism $\Psi_{2}$ of the ambient groups, which is defined over $k_{2}$, the trace field of $M_{2}$.

Now changing the models of $M_{1}$ and $M_{2}$ in an extension $\phi$ of $\varphi$ amounts to replacing the induced isomorphism $\Phi$ with $\Psi_{1} \circ \Phi \circ \Psi_{2}^{-1}$, for $\Psi_{1}, \Psi_{2}$ defined as above. Since $\Psi_{1}$ and $\Psi_{2}$ are defined over the trace fields $k_{1}$ and $k_{2}$ of $M_{1}$ and $M_{2}$ respectively, and since the field of definition of $\Phi$ contains (by definition) both $k_{1}$ and $k_{2}$, we see that it must equal the field of definition of $\Psi_{1} \circ \Phi \circ \Psi_{2}^{-1}$.

Therefore, for $i=1,2$, we can fix a model $\widetilde{M}_{i} \subset \mathbf{H}_{f_{i}}$ for $M_{i}$ with monodromy representation $\Gamma_{i} \subset \mathbf{P O}_{f_{i}}\left(k_{i}\right)$, and assume that both extensions $\phi$ and $\phi^{\prime}$ are maps $\mathbf{H}_{f_{1}} \rightarrow \mathbf{H}_{f_{2}}$. Moreover, since all hyperplane lifts $R_{i} \subset \partial \widetilde{M}_{i}$ of $N_{i} \subset \partial M_{i}$ are in the same $\Gamma_{i}$ orbit, we can assume (up to replacing $\phi^{\prime}$ by $\gamma_{2} \circ \phi^{\prime} \circ \gamma_{1}$ for some $\gamma_{1} \in \Gamma_{1}$ and $\gamma_{2} \in \Gamma_{2}$ ) that there are hyperplane lifts $R_{1} \subset \partial M_{1}$ of $N_{1}$ and $R_{2} \subset \partial M_{2}$ of $N_{2}$ such that both $\phi$ and $\phi^{\prime}$ send $R_{1}$ to $R_{2}$. Observe that since conjugation by $\gamma_{i} \in \Gamma_{i}$ is an operation defined over $k_{i}$, the same argument as before implies that we do not change the field of definition of $\Phi^{\prime}$.

Now since both $\phi$ and $\phi^{\prime}$ are extensions of the same $\varphi: N_{1} \rightarrow N_{2}$, the isometry $\phi^{-1} \circ \phi^{\prime}$ fixes $R_{1}$, and induces the identity isometry $N_{1} \rightarrow N_{1}$. By Proposition 2.17 we thus have that $\phi^{-1} \circ \phi^{\prime}=\gamma$ or $\rho_{1} \gamma$, where $\gamma \in \operatorname{Stab}_{\Gamma_{1}}\left(R_{1}\right)$ and $\rho_{1}$ is the reflection about $R_{1}$. Since $\gamma$ fixes $R_{1}$, we can again replace $\phi^{\prime}$ with $\phi^{\prime} \circ \gamma^{-1}$ (as before without changing the field of definition of $\Phi^{\prime}$ ) to obtain

$$
\phi^{-1} \circ \phi^{\prime} \in\left\{1, \rho_{1}\right\} .
$$

It follows that

$$
\Phi^{\prime}=\Phi \circ \Psi, \quad \Psi \in\left\{1, \text { conjugation by } \rho_{1}\right\} .
$$

Since $\rho_{1} \in \mathbf{P O}_{f_{1}}\left(k_{1}\right)$ by Proposition 3.9 we see that the fields of definition of $\Phi$ and $\Phi^{\prime}$ coincide, as desired.

4.3.2 The trace field of gluings. The goal of this section is to prove the main theorem of this thesis:

Theorem 4.7. Let $M$ be a manifold of type I constructed either by interbreeding $M_{1}$ and $M_{2}$, or closing up $M_{1}$. Let $\varphi$ denote the corresponding gluing isometry. Then the trace field of $M$ coincides with the field of definition of $\varphi$. 
In what follows we will often blur the distinction between a piece $M_{1}$ and its interior. In particular, we will see a piece of a manifold as embedded in it, although the boundary components may be identified. The following (easy) lemma allows us to adapt the model of a piece to that of the manifold which contains it, in a way that respects the hypersurfaces.

Lemma 4.8. Let $M$ be a manifold of type $I$, let $j: M_{1} \rightarrow M$ be an embedding of (the interior of) $M_{1}$ as a piece of $M$ and let $N \subset M_{1}$ be a hypersurface. Let $\widetilde{M}_{1} \subset \mathbf{H}_{f_{1}}$ be a model for $M_{1}$ with monodromy representation $\Gamma_{1} \subset \mathbf{P O}_{f_{1}}$, and let $\widetilde{M} \subset \mathbf{H}_{f}, \Gamma \subset \mathbf{P O}_{f}$ be defined similarly for $M$. Let $R_{1} \subset \widetilde{M}_{1}$ (resp. $R \subset \widetilde{M})$ be a hyperplane lift of $N$ (resp. $j(N))$. Then there exists an isometry $\phi: \mathbf{H}_{f_{1}} \rightarrow \mathbf{H}_{f}$ such that the diagram of Figure 4.1 commutes. In particular, if $\Phi: \mathbf{P O}_{f_{1}, \mathbb{R}} \rightarrow \mathbf{P O}_{f, \mathbb{R}}$ denotes the isomorphism induced by $\phi$, we have

$$
\Phi\left(\Gamma_{1}\right)=\operatorname{Stab}_{\Gamma}\left(\phi\left(\widetilde{M}_{1}\right)\right) .
$$

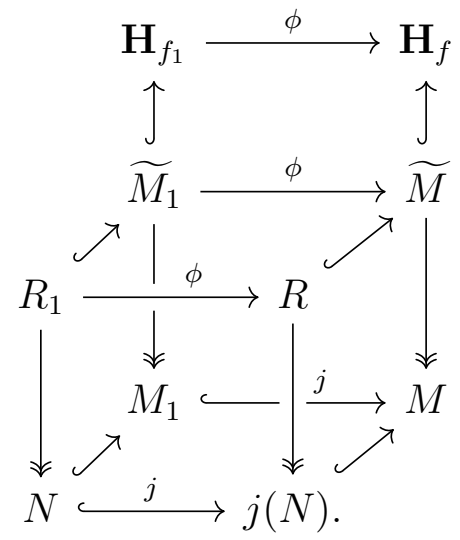

Figure 4.1: A "chair" diagram.

Proof. Let $\widetilde{M_{1}^{\prime}}$ be a connected component of the pre-image of $j\left(M_{1}\right) \subset M$ under the covering $\widetilde{M} \rightarrow M$. Then the induced map $\widetilde{M}_{1}^{\prime} \rightarrow M_{1}$ is a universal covering of (the interior of) $M_{1}$, and it follows that there exists an isometry $\phi: \widetilde{M}_{1} \rightarrow \widetilde{M_{1}^{\prime}} \subset \widetilde{M}$ which induces the embedding $j: M_{1} \rightarrow M$. Since these are intersection of half-spaces, $\phi$ is the restriction of an isometry $\phi: \mathbf{H}_{f_{1}} \rightarrow \mathbf{H}_{f}$. As the choice of $\widetilde{M}_{1}^{\prime}$ was arbitrary, we can assume that $R \subset \widetilde{M}_{1}^{\prime}$. Now $\phi^{-1}(R)$ is a hyperplane lift of $N$, and hence there exists an element $\gamma_{1} \in \Gamma_{1}$ such that $\gamma_{1}\left(R_{1}\right)=\phi^{-1}(R)$. Thus upon replacing $\phi$ with $\phi \circ \gamma_{1}$, we may assume that $\phi\left(R_{1}\right)=R$. Since $\phi$ induces $j$ on $M_{1}$, it also induces $j: N \rightarrow j(N)$.

Looking at the group of deck transformations of $\widetilde{M_{1}^{\prime}} \rightarrow j\left(M_{1}\right)$, we see that it equals $\operatorname{Stab}_{\Gamma}\left(\widetilde{M}_{1}^{\prime}\right)$. But since $\widetilde{M}_{1}^{\prime}=\phi\left(\widetilde{M}_{1}\right)$, it must also equal $\Phi\left(\Gamma_{1}\right)$, completing the proof. 
Corollary 4.9. Let $k_{1}$ (resp. $k$ ) denote the trace field of $M_{1}$ (resp. M). Then $k_{1} \subset k$ and the isomorphism $\Phi$ is defined over $k$, i.e., is an isomorphism of $k$-groups $\Phi: \mathbf{P O}_{f_{1}, k} \rightarrow \mathbf{P O}_{f}$.

Proof. We have $\Phi\left(\Gamma_{1}\right) \subset \Gamma \subset \mathbf{P O}_{f}(k)$. By minimality of the trace field, we obtain $k_{1} \subset k$. Moreover, since $\Gamma_{1} \subset \mathbf{P O}_{f_{1}}\left(k_{1}\right)$ is (almost) Zariski-dense, Proposition 2.13 implies that $\Phi$ is defined over $k$.

Remark 4.3.3. It follows that the ambient group of any gluing is always a base extension of the ambient group of any of its pieces.

We are ready for the:

Proof of Theorem 4.7. We first consider the interbreeding case. For $i=1,2$, let $\widetilde{M}_{i} \subset \mathbf{H}_{f_{i}}$ be a model for $M_{i}$, and let $\widetilde{M} \subset \mathbf{H}_{f}$ be one for $M$. Let $k_{i}$ (resp. $k$ ) denote the trace field of $M_{i}$ (resp. $M$ ) and let $\Gamma_{i} \subset \mathbf{P O}_{f_{i}}\left(k_{i}\right)$ (resp. $\left.\Gamma \subset \mathbf{P O}_{f}(k)\right)$ be a monodromy representation for $M_{i}(\operatorname{resp} . M)$.

As the (interior of the) manifold $M_{1}$ naturally embeds in $M$, Lemma 4.8 and its corollary imply that $k_{1} \subset k$ and that we can assume $f=f_{1}, \widetilde{M}_{1} \subset \widetilde{M}$, $\Gamma \subset \mathbf{P O}_{f_{1}}(k)$ and $\Gamma_{1}=\operatorname{Stab}_{\Gamma}\left(\widetilde{M}_{1}\right)$. Applying the same argument to the embedding $M_{2} \hookrightarrow M$ gives that $k_{2} \subset k$ and we have an isometry $\phi: \mathbf{H}_{f_{2}} \rightarrow \mathbf{H}_{f_{1}}$ such that $\Phi\left(\Gamma_{2}\right)=\operatorname{Stab}_{\Gamma}\left(\phi\left(\widetilde{M}_{2}\right)\right)$, where $\Phi$ is the isomorphism induced by $\phi$.

Let $N_{i} \subset M_{i}(i=1,2)$ denote the gluing hypersurface, and let $N \subset M$ denote the same hypersurface seen in $M$. Let $R_{1} \subset \widetilde{M}_{1} \subset \widetilde{M}$ be a hyperplane lift of $N_{1}$ (and of $N$ ), and let $R_{2} \subset \widetilde{M}_{2}$ be one of $N_{2}$. It follows from Lemma 4.8 that we may choose $\phi$ such that $\phi\left(R_{2}\right)=R_{1}$.

Let $\varphi: N_{2} \rightarrow N_{1}$ denote the gluing isometry. By construction, $\phi$ is an extension of $\varphi$. Let $\ell$ denote the field of definition of $\varphi$; it is the field of definition of the algebraic isomorphism $\Phi: \mathbf{P O}_{f_{2}, \mathbb{R}} \rightarrow \mathbf{P O}_{f_{1}, \mathbb{R}}$. Note that by definition, $\ell$ contains $k_{1}$ and $k_{2}$. Observe that since $\pi_{1}(M)$ is generated by the images of $\pi_{1}\left(M_{1}\right)$ and $\pi_{1}\left(M_{2}\right)$ under the natural maps induced via inclusion $M_{i} \hookrightarrow M$, it follows that $\Gamma=\left\langle\Gamma_{1}, \Phi\left(\Gamma_{2}\right)\right\rangle$. Since $\Gamma_{1} \subset \mathbf{P O}_{f_{1}}\left(k_{1}\right) \subset \mathbf{P O}_{f_{1}}(\ell)$ and $\Phi\left(\Gamma_{2}\right) \subset \Phi\left(\mathbf{P O}_{f_{2}}\left(k_{2}\right)\right) \subset \mathbf{P} \mathbf{O}_{f_{1}}(\ell)$, we see that the trace field $k$ of $\Gamma$ must be contained in $\ell$. On the other hand, since $\Phi$ sends $\Gamma_{2} \subset \mathbf{P O}_{f_{2}}\left(k_{2}\right) \subset \mathbf{P O}_{f_{2}}(k)$ into $\Gamma \subset \mathbf{P O}_{f_{1}}(k)$, it follows from Proposition 2.13 that $\Phi$ is defined over $k$, i.e., $\ell \subset k$. This completes the proof in the interbreeding case.

We now consider the closing up case; we will use the same notation for $M$, $M_{1}$ as in the interbreeding case. As before we have $k_{1} \subset k$ and we can assume (using Lemma 4.8) that $f=f_{1}, \widetilde{M}_{1} \subset \widetilde{M}, \Gamma \subset \mathbf{P O}_{f_{1}}(k)$ and $\Gamma_{1}=\operatorname{Stab}_{\Gamma}\left(\widetilde{M}_{1}\right)$. We let $N_{1}, N_{2}$ denote the two hypersurfaces in $\partial M_{1}, N$ the corresponding hypersurface in $M, R_{1}$ a hyperplane lift of both $N_{1}$ and $N$, and $R_{2}$ one of $N_{2}$. The fundamental group $\pi_{1}(M)$ is now generated by $\pi_{1}\left(M_{1}\right)$ and a loop $\lambda$ starting on one side of $N$ and ending on the other side. Let $\eta \in \Gamma$ be the isometry corresponding to $\lambda$; it follows that $\eta R_{1}$ is a hyperplane lift of $N_{2}$. 
Since those are conjugate under the action of $\Gamma_{1}$, there is a $\gamma_{1} \in \Gamma_{1}$ such that $\gamma_{1} \eta R_{1}=R_{2}$. Set $\phi=\gamma_{1} \eta$. We have $\Gamma=\left\langle\Gamma_{1}, \phi\right\rangle$.

Again as before, $\phi$ is an extension of $\varphi$. Let $\ell$ denote the field of definition of $\varphi$, i.e., the field of definition of the induced (algebraic) automorphism $\Phi: \mathbf{P O}_{f_{1}, \mathbb{R}} \rightarrow \mathbf{P} \mathbf{O}_{f_{1}, \mathbb{R}}$. Since $\Phi$ is conjugation by $\phi \in \Gamma \subset \mathbf{P} \mathbf{O}_{f_{1}}(k)$, we have $\ell \subset k$. Moreover, Theorem 2.11 implies that $\phi \in \mathbf{P O}_{f_{1}}(\ell)$ and since $k_{1} \subset \ell$ and $\Gamma \subset \mathbf{P O}_{f_{1}}\left(k_{1}\right)$ we have $k \subset \ell$ and the proof is complete.

\section{4 $\mid$ Corollaries and computations}

In this section we explore some by-products of Theorem 4.7 and compute the trace field of the known constructions of nonarithmetic manifolds.

4.4.1 Gluing and quadratic extensions. The first corollary of Theorem 4.7 we will see is the following.

Theorem 4.10. Let $M$ be obtained either by interbreeding the manifolds $M_{1}$ and $M_{2}$ or by closing up $M_{1}$. Let $k$ denote the composite of the trace fields of $M_{1}$ and $M_{2}$ in the interbreeding case, and the trace field of $M_{1}$ in the closing up case. Then the trace field of $M$ is an extension of $k$ that is at most quadratic.

Proof. We treat both cases at once by setting $M_{2}=M_{1}$ in the closing up case. Let $\widetilde{M}_{i} \subset \mathbf{H}_{f_{i}}$ be a model for $M_{i}$, and let $N_{i} \subset M_{i}$ denote the gluing hypersurface. Up to choosing a different orthogonal basis for $f_{i}$, we may assume that $f_{i}=q_{i}+a_{i} x_{n}^{2}$ for some quadratic form $q_{i}$ and that the hyperplane $R_{i} \subset \mathbf{H}_{f_{i}}$ corresponding to $\left\{x_{n}=0\right\}$ is a hyperplane lift of $N_{i} \subset M_{i}$. By Lemma 3.12, the isometry $\varphi: N_{1} \rightarrow N_{2}$ is induced by a matrix $A_{0} \in \mathrm{GL}_{n}(k)$ such that:

$$
q_{2} \circ A_{0}=\lambda q_{1}, \quad \text { for some } \lambda \in k .
$$

Thus up to replacing $f_{2}$ with $\lambda^{-1}\left(f_{2} \circ A\right)=q_{1}+\lambda^{-1} a_{2} x_{n}^{2}$, where

$$
A=\left(\begin{array}{cc}
A_{0} & 0 \\
0 & 1
\end{array}\right)
$$

and conjugating the monodromy representations accordingly, we can assume that $f_{2}=q_{1}+a_{2} x_{n}^{2}$. Now by construction, using these models, the matrix

$$
B=\left(\begin{array}{cc}
I & 0 \\
0 & \sqrt{a_{1} / a_{2}}
\end{array}\right)
$$

is such that $f_{2} \circ B=f_{1}$, and thus induces an isometry $\phi: \mathbf{H}_{f_{1}} \rightarrow \mathbf{H}_{f_{2}}$ which is an extension of the original gluing isometry $\varphi$. Therefore the trace field of $M$ is contained in $k\left(\sqrt{a_{1} / a_{2}}\right)=k\left(\sqrt{a_{1} a_{2}}\right)$, as desired. 
4.4.2 Doubling and trace field. The next result is about doubles of manifolds with boundary. Recall that if $M$ is a manifold of type I, its double is the manifold $M \times\{0,1\} / \sim$, where $(x, 0) \sim(x, 1)$ for $x \in \partial M$ (see Definition 2.2.7). If $N_{1}, \ldots, N_{r}$ are a subset of the boundary hypersurfaces of $M$, one can also consider the (restricted) double of $M$ at $N_{1}, \ldots, N_{r}$, which can be defined as $M \times\{0,1\} / \sim$ where $(x, 0) \sim(x, 1)$ only for $x \in N_{1} \cup \cdots \cup N_{r}$.

Proposition 4.11. The double of a manifold $M$ of type $I$ along any number of components in $\partial M$ has the same trace field as $M$.

Proof. Let $N_{1}, \ldots, N_{r} \subset \partial M$ be the boundary components at which we want to form the double $M^{\prime}$. Then $M^{\prime}$ can be constructed as follows:

1. Form the interbreeding of $M$ with a copy of itself at $N_{1}$ (with trivial gluing isometry); call the resulting manifold $M_{1}$.

2. If $M_{i-1}$ has been defined, construct $M_{i}$ by closing up $M_{i-1}$ at the two boundary components corresponding to $N_{i}$ (with trivial gluing isometry).

The manifold $M_{r}$ is the double of $M$ we wanted to construct. We will show that each step of this construction does not change the trace field.

Let $\widetilde{M} \subset \mathbf{H}_{f}$ be a model for $M$ with corresponding monodromy representation $\Gamma \subset \mathbf{P O}_{f}(k)$, where $k$ is the trace field of $M$. Choose the same model for the other copy of $M$ used to construct $M_{1}$. By considering the same hyperplane lifts in both cases, one sees that the identity isometry id $\in \mathbf{P O}_{f}(k)$ is an extension of the gluing isometry of $M_{1}$. Thus $M_{1}$ has the same trace field as $M$.

Assume $M_{i}$ has been constructed, and choose a model $\widetilde{M}_{i} \subset \mathbf{H}_{f}$ for $M_{i}$ with monodromy representation $\Gamma_{i} \subset \mathbf{P O}_{f}(k)$ such that $\widetilde{M} \subset \widetilde{M}_{i}$ and

$$
\Gamma=\operatorname{Stab}_{\Gamma_{i}}(\widetilde{M}) \text {. }
$$

Let $N_{i+1}, N_{i+1}^{\prime}$ denote the two copies of $N_{i+1}$ in $\partial M_{i}$.

By construction, reflection at $N_{1} \subset M_{i}$ induces an isometry of $M_{i}$; call it $\rho_{1}$. Observe that $\rho_{1} N_{i+1}=N_{i+1}^{\prime}$. Composing the covering map $\widetilde{M}_{i} \rightarrow M_{i}$ with $\rho_{1}$, we obtain another model $\widetilde{M}_{i}^{\prime} \subset \mathbf{H}_{f}$ with monodromy representation $\Gamma_{i}^{\prime} \subset \mathbf{P O}_{f}(k)$ such that $\widetilde{M}_{i}^{\prime}=\widetilde{M}_{i}$ and $\Gamma_{i}^{\prime}=\rho_{1} \Gamma_{i} \rho_{1} \neq \Gamma_{i}$. Since $\rho_{1} N_{i+1}=N_{i+1}^{\prime}$, we see that we can choose a hyperplane $R \subset \mathbf{H}_{f}$ such that $R \subset \widetilde{M}_{i}$ is a hyperplane lift of $N_{i+1}$ and $R \subset \widetilde{M}_{i}^{\prime}$ is a hyperplane lift of $N_{i+1}^{\prime}$. There again, id $\in \mathbf{P O}_{f}(k)$ is an extension of the gluing isometry, and so $M_{i+1}$ still has the same trace field as $M_{i}$.

Remark 4.4.1. Proposition 4.11 can also be deduced as a corollary of Proposition 3.9. Indeed, the orbifold defined by $M$ with "mirrors" at the boundary components has the same trace field as $M$ by Proposition 3.9 (since it has a monodromy representation given by $\left\langle\Gamma, \rho_{1}, \ldots, \rho_{r}\right\rangle$, where $\Gamma$ is one for $M$ and the $\rho_{i}$ are the reflections at hyperplane lifts of the $N_{i}$ ). The double of $M$ is then a double cover of this orbifold. 
4.4.3 Odd dimensional closing up. The third proposition of this section tells us that in odd dimension, closing up does not change the trace field.

Proposition 4.12. Let $M$ be an n-manifold of type I. If the dimension $n$ is odd, then closing up $M$ at any subset of its boundary components does not change the trace field.

Proof. Let $\widetilde{M} \subset \mathbf{H}_{f}$ be a model for $M$ with corresponding monodromy representation $\Gamma \subset \mathbf{P O}_{f}(k)$, where $k$ is the trace field of $M$. Let $N_{1}, N_{2}$ be two isometric boundary components of $M$ with hyperplane lifts $R_{1}, R_{2} \subset \partial \widetilde{M}$ and let $\varphi: N_{1} \rightarrow N_{2}$ be a gluing isometry. Let $\phi \in \mathbf{P O}_{f}(\mathbb{R})$ be an extension of $\varphi$ such that $\phi\left(R_{1}\right)=R_{2}$.

Let $\Phi: \mathbf{P O}_{f, \mathbb{R}} \rightarrow \mathbf{P} \mathbf{O}_{f, \mathbb{R}}$ be the map induced via conjugation by $\phi$ and let $\sigma$ be a $k$-automorphism of $\mathbb{C}$. Then ${ }^{\sigma} \Phi$ is induced via conjugation by $\sigma(\phi)$. Let $\mathbf{G}_{0}$ denote the Zariski-closure of $\operatorname{Stab}_{\Gamma}(R)$ in $\mathbf{P O}_{f}$. As $\operatorname{Stab}_{\Gamma}\left(R_{1}\right) \subset \mathbf{P O}_{f}(k)$, the maps $\Phi$ and ${ }^{\sigma} \Phi$ must agree on it. Therefore, $\Phi^{-1} \circ{ }^{\sigma} \Phi$ is the identity on $\operatorname{Stab}_{\Gamma}\left(R_{1}\right)$, and thus also on $\mathbf{G}_{0}$ by Zariski-density. It follows that $\phi^{-1} \sigma(\phi)$ commutes with every element of $\mathbf{G}_{0}$. By Lemma 3.10 (or more precisely the argument in the proof of Proposition 3.9), we have $\sigma(\phi)=\phi$ or $\sigma(\phi)=\phi \rho$ (where $\rho$ is the reflection at $R_{1}$ ). Moreover, since $\rho \in \mathbf{P O}_{f}(k)$ by Proposition 3.9, we have $\sigma(\phi \rho)=\sigma(\phi) \rho$ and thus $\sigma$ acts on the set $\{\phi, \phi \rho\}$.

Since $n$ is odd, the algebraic group $\mathbf{P} \mathbf{O}_{f}$ has two connected components, and $\mathbf{P O}_{f}^{\circ}(\mathbb{R})$ consists of the orientation-preserving isometries of $\mathbf{H}_{f}$. It follows that the set $\mathbf{P} \mathbf{O}_{f}^{\circ} \cap\{\phi, \phi \rho\}$ contains exactly one element. Now as $\mathbf{P O}_{f}^{\circ}$ is defined over $k$, it is stable under $\sigma$, and thus $\sigma$ fixes one, and hence both elements of $\{\phi, \phi \rho\}$, i.e., $\sigma(\phi)=\phi$. Since $\sigma$ was arbitrary, Lemma 2.1 implies that $\phi \in \mathbf{P O}_{f}(k)$. Since by construction, $\phi$ was an extension of the gluing isometry $\varphi$, the trace field of the closing up of $M$ is $k$ and the proof is complete.

4.4.4 Gluings of arithmetic pieces. As explained in Section 4.2.1, the gluing isometries in Gromov and Piatetski-Shapiro's constructions and their generalizations are usually not specified, and thus the trace field cannot be computed as such. However, there is always a canonical isometry one can use (see Definition 4.2.1). When gluing arithmetic pieces with this isometry, the trace field of the resulting gluing can be computed easily using Theorem 4.7.

Theorem 4.13. Let $M \in \mathcal{A}^{g l}$ be a gluing of arithmetic pieces. Assume the pieces used in the construction are arithmetic pieces of trace field $k$ and type $\left(f_{i}, \mathfrak{a}\right)$ or $\left(f_{i}, \mathfrak{a}, m_{i}\right)$ (see Definition 4.1.6), for $i=1, \ldots, r$, where $f_{i}=f_{0}+a_{i} x_{n}^{2}$, and that the gluing isometries are the canonical ones. Then the trace field of $M$ is

$$
k\left(\sqrt{a_{i} a_{j}} \mid 1 \leq i, j \leq r\right)=k\left(\sqrt{a_{1} a_{2}}, \ldots, \sqrt{a_{1} a_{r}}\right) .
$$

Proof. By definition, there exists a sequence $M_{0}, \ldots, M_{s}$ where $M_{0} \in \mathcal{A}$ is an arithmetic piece, $M_{s}=M$ and $M_{i+1}$ is constructed from $M_{i}$ by interbreeding 
$M_{i}$ with a piece in $\mathcal{A}$ or closing up $M_{i}$. Assume first that $M_{i+1}$ is constructed by interbreeding $M_{i}$ with an arithmetic piece $A_{1}$ of coming from the quadratic form $f_{1}=f_{0}+a_{1} x_{n}^{2}$. Let $k_{i}$ denote the trace field of $M_{i}$. Let $A_{2}$ be the arithmetic piece of $M_{i}$ containing the boundary hypersurface to which $A_{1}$ is glued, and assume $A_{2}$ comes from the quadratic form $f_{2}=f_{0}+a_{2} x_{n}^{2}$. For $j=1,2$, let $N_{j} \subset A_{j}$ be the gluing hypersurfaces in the boundary. By definition, there are models $\widetilde{A}_{j} \subset \mathbf{H}_{f_{j}}$ for $A_{j}$ with monodromy representation $\Lambda_{j} \subset \mathbf{P O}_{f_{j}}(k)$ such that the hyperplane defined by $\left\{x_{n}=0\right\}$ is a hyperplane lift of $N_{j} \subset A_{j}$. By Lemma 4.8 , there is a model $\widetilde{M}_{i} \subset \mathbf{H}_{f_{1}}$ with monodromy representation $\Gamma_{i} \subset \mathbf{P O}_{f_{1}}\left(k_{i}\right)$ such that

$$
\widetilde{A}_{1} \subset \widetilde{M}_{i} \text { and } \quad \Lambda_{1}=\operatorname{Stab}_{\Gamma_{i}}\left(\widetilde{A}_{1}\right)
$$

Now by hypothesis, the gluing isometry is the canonical one, and thus an extension is given by

$$
\begin{aligned}
\phi: \mathbf{H}_{f_{1}} & \longrightarrow \mathbf{H}_{f_{2}} \\
x & \longmapsto\left(\begin{array}{cc}
I & 0 \\
0 & \sqrt{a_{1} / a_{2}}
\end{array}\right) x .
\end{aligned}
$$

The field of definition of the induced isomorphism

$$
\Phi: \mathbf{P O}_{f_{1}, k_{i}} \longrightarrow \mathbf{P O}_{f_{2}, k_{i}}
$$

then is $k_{i}\left(\sqrt{a_{1} / a_{2}}\right)=k_{i}\left(\sqrt{a_{1} a_{2}}\right)$. Indeed, if $\sqrt{a_{1} a_{2}} \in k_{i}$ there is nothing to do, otherwise the unique non-trivial $k_{i}$-automorphism of $k_{i}\left(\sqrt{a_{1} a_{2}}\right)$ sends $\Phi$ to $\rho \circ \Phi$, where $\rho$ denotes the reflection at the hyperplane defined by $\left\{x_{n}=0\right\}$, and thus $\Phi$ is not defined over $k_{i}$, but over $k_{i}\left(\sqrt{a_{1} a_{2}}\right)$.

Assume now that $M_{i+1}$ is the closing up of $M_{i}$. As before, let $A_{1}, A_{2}$ denote the arithmetic pieces where the gluing happens, allowing the case $A_{1}=A_{2}$. Then the proof proceeds as in the interbreeding case, choosing models in $\mathbf{H}_{f_{j}}$ where hyperplane lifts of the gluing hypersurfaces are $\left\{x_{n}=0\right\}$, and using the above map as an extension. Observe that in case $A_{1}=A_{2}$, the hyperplane $\left\{x_{n}=0\right\}$ is a lift of both hypersurfaces and thus the identity is a valid extension.

By induction we have that the trace field of $M$ is

$$
k_{r}=k\left(\sqrt{a_{i_{1}} a_{i_{2}}}, \ldots, \sqrt{a_{i_{2 r-1}} a_{i_{2 r}}}\right),
$$

where the sequence $i_{1}, \ldots, i_{2 r}$ depends on the gluing ordering. Since all pieces are used, this sequence contains all indices between 1 and $r$, and thus $k_{r}$ is easily seen to be the field given in the statement of the theorem.

It is interesting to compare this result to Theorem 4.10, where the isometries are not specified and thus the quadratic extension is not explicit. Applying Theorem 4.13 to the case of the gluings of Gromov and Piatetski-Shapiro with canonical gluing isometries, we obtain: 
Corollary 4.14. Let $M$ be a gluing of Gromov and Piatetski-Shapiro (as described in Section 4.2.1). Assume the gluing isometry is the canonical one. Let $f_{i}=f_{0}+a_{i} x_{n}^{2},(i=1,2)$ be the two quadratic forms over $k$ used to define the pieces. Then the trace field of $M$ is $k\left(\sqrt{a_{1} / a_{2}}\right)=k\left(\sqrt{a_{1} a_{2}}\right)$.

Remark 4.4.2. Since the quadratic forms $f_{i}$ are chosen to be non-similar (so that the arithmetic groups are non-commensurable, c.f. Theorem 2.24), $a_{1} / a_{2}$ cannot be a square in $k$. Thus the trace field $K$ of the gluing is a quadratic extension of $k$. However, since its ambient group is $\mathbf{G}=\mathbf{P O}_{f_{1}, K}=\mathbf{P O}_{f_{2}, K}$ (see Remark 4.3.3) and $\mathbf{P} \mathbf{O}_{f_{1}}$ is admissible over $k, \mathbf{G}$ cannot be admissible over $K$ (see also Remark 5.1.2 below). This gives another proof that these gluings are nonarithmetic.

Similarly, the trace fields of the gluings of Gelander and Levit (assuming the canonical gluing isometry) is an easy consequence of Theorem 4.13:

Corollary 4.15. Let $M_{\Delta}$ be a gluing of Gelander and Levit (as described in Section 4.2.2). Assume the gluing isometries are the canonical ones. Let $f_{i}=f_{0}+a_{i} x_{n}^{2},(i=1, \ldots, 6)$ be the quadratic forms over $k$ used to define the pieces. Then the trace field of $M_{\Delta}$ is

$$
k\left(\sqrt{a_{i} / a_{j}} \mid i, j=1, \ldots, 6\right)=k\left(\sqrt{a_{1} a_{2}}, \ldots, \sqrt{a_{1} a_{6}}\right) .
$$

\subsection{Gluing commensurable manifolds}

In this section, we will see how we can use Theorem 4.13 to construct gluings of pieces of commensurable manifolds of trace field $k$, whose trace field is an arbitrary large extension of $k$. We will also give examples of arithmetic pieces whose trace field increases under closing up.

4.5.1 Gromov-Piatetski-Shapiro revisited. The goal of this section is to study what happens when the gluing construction of Gromov and Piatetski-Shapiro and its generalizations (see Section 4.2.1) are applied with pieces of commensurable arithmetic manifolds. We will need a small lemma about quadratic forms of a given shape.

Lemma 4.16. Let $f_{0}$ be a quadratic form over a number field field $k$ and for $i=1,2$ let $a_{i} \in k^{\times}$and $f_{i}=f_{0} \perp\left\langle a_{i}\right\rangle=f_{0}+a_{i} y^{2}$. Then the following are equivalent:

1. $f_{1}$ and $f_{2}$ are similar (i.e. $\exists \lambda \in k$ such that $f_{1}$ is isometric to $\lambda f_{2}$ ).

2. $a_{1} f_{0}$ is isometric to $a_{2} f_{0}$. 
Proof. If 2. holds, then $a_{2} f_{1}=a_{2} f_{0}+a_{1} a_{2} y^{2} \cong a_{1} f_{0}+a_{1} a_{2} y^{2}=a_{1} f_{2}$, which implies similarity with factor $a_{1} / a_{2}$. If 1 . holds, let $\lambda \in k^{\times}$be such that $f_{1} \cong \lambda f_{2}$. If the dimension of the $f_{i}$ is even, the discriminant of $f_{2}$ equals that of $\lambda f_{2}$. Therefore $f_{1} \cong \lambda f_{2}$ implies that $a_{1} \equiv a_{2} \bmod \left(k^{\times}\right)^{2}$, i.e., $a_{1} / a_{2}$ is a square in $k$. It follows that $a_{1} f_{0} \cong a_{2} f_{0}$. In that case, $f_{1}$ and $f_{2}$ are even isometric.

If the dimension is odd, again equating the discriminants gives $\lambda=a_{1} / a_{2}$ $\bmod \left(k^{\times}\right)^{2}$. Therefore $f_{0}+a_{1} y^{2}=f_{1} \cong\left(a_{1} / a_{2}\right) f_{2}=\left(a_{1} / a_{2}\right) f_{0}+a_{1} y^{2}$, and by Witt cancellation we have $f_{0} \cong\left(a_{1} / a_{2}\right) f_{0}$, i.e., $a_{2} f_{0} \cong a_{1} f_{0}$, as desired.

Using this lemma, one can apply the Gromov-Piatetski-Shapiro construction with pieces of arithmetic groups coming from similar (but not isomorphic) quadratic forms over $\mathbb{Q}$ and obtain interesting examples of gluings of pieces of commensurable manifolds. We can then use Theorem 4.13 to show that the trace fields we obtain can be arbitrary large extensions of $\mathbb{Q}$.

Corollary 4.17. Let $F$ be a totally real multiquadratic number field. Then for $n \equiv 2(\bmod 4)$ there exists an $n$-manifold $M$ which trace field $F$ which is obtained as a gluing of pieces of pairwise commensurable arithmetic manifolds with trace field $\mathbb{Q}$.

Proof. By hypothesis, there are positive elements $a_{1}, \ldots, a_{r} \in \mathbb{Q}^{\times}$such that $F=\mathbb{Q}\left(\sqrt{a_{1}}, \ldots, \sqrt{a_{r}}\right)$. Consider the quadratic forms

$$
f_{0}=-x_{0}^{2}+\cdots+x_{n-1}^{2} \quad \text { and } \quad f_{i}=f_{0}+a_{i} x_{n}^{2} .
$$

Theorem 4.13 implies the existence of a gluing $M$ with trace field $F$ constructed using pieces of arithmetic manifolds corresponding to the quadratic forms $f_{i}$. Thus we only need to show that all these arithmetic groups are commensurable, i.e., that all the forms $f_{i}$ are similar.

By Lemma 4.16, it suffices to show that $a f_{0} \cong f_{0}$ for any positive $a \in \mathbb{Q}$. The discriminants of these forms coincide, since the number of variables is even. Thus by the Hasse-Minkowski principle (see [Se77, IV.3.3]), it is enough to show that their Hasse invariants $\epsilon_{p}$ coincide for each prime $p$. Setting $\delta_{n}=-1$ and $\delta_{i}=1$ otherwise, we have, for any prime $p$ :

$$
\epsilon_{p}\left(a f_{0}\right)=\prod_{i<j}\left(a \delta_{i}, a \delta_{j}\right)_{p}=(a, a)_{p}^{\frac{(n-1)(n-2)}{2}} \cdot(a,-a)_{p}^{n-1}
$$

where $(\cdot, \cdot)_{p}$ denotes the Hilbert symbol at $p$. The term $(a,-a)_{p}$ equals one by standard properties (see [Se77, III.1.1]), and since $n \equiv 2(\bmod 4)$, the quantity $\frac{(n-1)(n-2)}{2}$ is even. Therefore $\epsilon_{p}\left(a f_{0}\right)=1=\epsilon_{p}\left(f_{0}\right)$ for any prime $p$ and any $a$.

If one starts with an admissible quadratic form $f_{0}$ over a totally real number field $k$ such that $a_{i} f_{0} \cong f_{0}$ for totally positive elements $a_{i} \in k, i=1, \ldots, r$, then the same construction will give a manifold with trace field $k\left(\sqrt{a_{1}}, \ldots, \sqrt{a_{r}}\right)$. 
4.5.2 Closing up arithmetic pieces. As shown in Proposition 4.12 the operation of closing up a manifold does not change the trace field provided its dimension is odd. The goal of this section is to give examples of arithmetic pieces of even dimension, where the trace field increases under closing up. We will give first a (relatively) general lemma about how to construct such examples and illustrate the method in the particular case of $k=\mathbb{Q}$.

Lemma 4.18. Let $f_{0}\left(x_{0}, \ldots, x_{n-1}\right)$ be an admissible quadratic form over a totally real number field $k$, and let $a \in k$ be totally positive and such that $f_{0} \cong a f_{0}$. Assume there exists a matrix $A_{0} \in \mathrm{GL}_{n}(k)$ such that

$$
\begin{aligned}
& \text { 1. } f_{0} \circ A_{0}=a f_{0}, \\
& \text { 2. } \frac{1}{a} A_{0}^{2} \in \mathbf{O}_{f_{0}}\left(\mathcal{O}_{k}\right) .
\end{aligned}
$$

Then there is a piece $M$ of the arithmetic manifold corresponding to $f=f_{0}+x_{n}^{2}$ and a gluing isometry $\varphi$ such that the closing up of $M$ under $\varphi$ has trace field $k(\sqrt{a})$.

Proof. We can assume that $a$ is not a square in $k$ (otherwise it is trivial). Let $\Gamma$ denote an arithmetic lattice corresponding to an arithmetic piece of type $(f, \mathfrak{a}, 1)$. This means that $\Gamma$ is a finite index subgroup of the image of $\mathbf{O}_{f}\left(\mathcal{O}_{k}\right)$ in $\mathbf{P O}_{f}$, chosen such that it is torsion-free and the hyperplane $R \subset \mathbf{H}_{f}$ corresponding to $\left\{x_{n}=0\right\}$ projects down to a non-separating hypersurface $N$ in $\Gamma \backslash \mathbf{H}_{f}$. Let $\Lambda=\operatorname{Stab}_{\Gamma}(R)$, so that $N=\Lambda \backslash R \cong \Lambda \backslash \mathbf{H}_{f_{0}}$.

The group $\Lambda$ is an arithmetic subgroup contained in the image of $\mathbf{O}_{f_{0}}\left(\mathcal{O}_{k}\right)$ in $\mathbf{P O}_{f}$. Let $\widetilde{\Lambda}$ be its pre-image in $\mathbf{O}_{f_{0}}\left(\mathcal{O}_{k}\right)$, and consider the matrix

$$
g_{0}=\frac{1}{\sqrt{a}} A_{0} .
$$

We have $g_{0}^{2} \in \mathbf{O}_{f_{0}}\left(\mathcal{O}_{k}\right)$ by hypothesis, and thus $g_{0}^{N} \in \widetilde{\Lambda}$ for a suitable power $N$ (since $\widetilde{\Lambda}$ is of finite index in $\mathbf{O}_{f_{0}}\left(\mathcal{O}_{k}\right)$ ). It follows that the group

$$
\widetilde{\Lambda}_{1}=\widetilde{\Lambda} \cap\left(g_{0} \widetilde{\Lambda} g_{0}^{-1}\right) \cap \cdots \cap\left(g_{0}^{N-1} \widetilde{\Lambda} g_{0}^{-N+1}\right)
$$

is a finite index subgroup of $\widetilde{\Lambda}$ that is normalized by $g_{0}$. Hence the image $\bar{g}_{0}$ of $g_{0}$ in $\mathbf{P O}_{f_{0}}$ induces an isometry of $N_{1}=\widetilde{\Lambda}_{1} \backslash \mathbf{H}_{f_{0}}$. Observe as a side note that $\bar{g}_{0} \in \mathbf{P O}_{f_{0}}(k)$, since $\sigma\left(g_{0}\right)=-g_{0}$ for the unique non trivial $k$-automorphism $\sigma$ of $k(\sqrt{a})$.

Let $\Lambda_{1}$ denote the image of $\widetilde{\Lambda}_{1}$ in $\mathbf{P O}$. Since $\Lambda$ is geometrically finite, so is $\Lambda_{1}$, hence it is separable in $\Gamma$ (Theorem 2.28). It follows that there exists a finite index subgroup $\Gamma_{1} \subset \Gamma$ such that $\operatorname{Stab}_{\Gamma_{1}}(R)=\Lambda \cap \Gamma_{1}=\Lambda_{1}$.

Consider now the matrix

$$
g=\left(\begin{array}{cc}
g_{0} & 0 \\
0 & 1
\end{array}\right)
$$


It preserves $f$, and thus defines an element $\bar{g}$ in $\mathbf{P} \mathbf{O}_{f}(k(\sqrt{a}))$. However, if as before $\sigma$ is the non-trivial $k$-automorphism of $k(\sqrt{a})$, we have $\sigma(\bar{g})=\bar{g} \rho$, where $\rho$ is the reflection at the hyperplane $R$, and thus $\bar{g} \notin \mathbf{P O}_{f}(k)$.

We are ready to complete the proof. Write $M_{1}=\Gamma_{1} \backslash \mathbf{H}_{f}, N_{1}=\Lambda_{1} \backslash R \subset M_{1}$ and let $M$ denote the completion of $M_{1} \backslash N_{1}$. Since $N_{1}$ is non-separating in $M_{1}, M$ is connected. Let $N_{2}, N_{3}$ denote the two boundary components of $M$ isometric to $N$. Let $M^{\prime}$ be obtained by closing up $M$ along the isometry $N_{2} \cong N_{3}$ induced by $\bar{g}_{0}$.

In order to compute the trace field of $M^{\prime}$, we consider the canonical models $\widetilde{M}_{2}, \widetilde{M}_{3} \subset \mathbf{H}_{f}$ for $M$ associated to the hypersurfaces $N_{2}$ and $N_{3}$ respectively. Recall that for such models, we have that $R \subset \partial \widetilde{M}_{i}$ is a hyperplane lift of $N_{i} \subset M$. It follows that, by construction, the element $\bar{g}$ is an extension of the gluing isometry. Since its field of definition is $k(\sqrt{a})$, the trace field of $M^{\prime}$ is also $k(\sqrt{a})$, as desired.

Remark 4.5.1. This construction can be seen as a refinement of the GromovPiatetski-Shapiro construction with commensurable pieces (as seen in the previous section). Indeed, the quadratic form $f$ from the lemma is similar to $f^{\prime}=f_{0}+a x_{n}^{2}$ by Lemma 4.16. Thus if one chooses two different models for $M$, one in $\mathbf{H}_{f}$ and one in $\mathbf{H}_{f^{\prime}}$, the following matrix can be chosen as an extension of the gluing isometry:

$$
g=\left(\begin{array}{cc}
1 & 0 \\
0 & \sqrt{a}
\end{array}\right)
$$

This gives directly that the gluing $M$ has trace field $k(\sqrt{a})$.

Note however that more efforts are needed to construct the piece used for closing up, since we have only this piece at our disposal and are not allowed to use ones coming from commensurable manifolds as in Theorem 4.13. In particular, finding a similitude $A_{0}$ satisfying condition 2 . from the lemma is crucial for our purpose (in order to have an arithmetic group normalized by the $g_{0}$ from above).

We end this section by giving explicit examples of pieces of manifolds whose closing up increases the trace field by a quadratic extension.

Example 4.5.2. We will use the notation $\left\langle a_{1}, \ldots, a_{m}\right\rangle$ for the quadratic form $a_{1} x_{1}^{2}+\cdots+a_{m} x_{m}^{2}$ and $\perp$ for orthogonal sum. Consider the following quadratic forms over $\mathbb{Q}$ :

$$
q_{1}=\langle-1,1,1,2\rangle \quad \text { and } \quad q_{2}=\langle 1,2\rangle .
$$

For $m \geq 0$, set

$$
f_{0}=q_{1} \perp \underbrace{q_{2} \perp \cdots \perp q_{2}}_{m \text { times }}, \quad \text { and } \quad f=f_{0} \perp\langle 1\rangle \text {. }
$$


Thus $f_{0}=\langle-1,1,1,2,1,2, \ldots, 1,2\rangle$ is an admissible quadratic form over $\mathbb{Q}$ of dimension $2 m+4$. Define the matrices

$$
A_{1}=\left(\begin{array}{cccc}
2 & 0 & 1 & 0 \\
0 & 1 & 0 & 2 \\
-1 & 0 & -2 & 0 \\
0 & 1 & 0 & -1
\end{array}\right) \quad \text { and } \quad A_{2}=\left(\begin{array}{cc}
1 & 2 \\
1 & -1
\end{array}\right)
$$

We have $q_{1} \circ A_{1}=3 \cdot q_{1}$ and $q_{2} \circ A_{2}=3 \cdot q_{2}$, and thus the matrix

$$
A_{0}=\left(\begin{array}{llll}
A_{1} & & & \\
& A_{2} & & \\
& & \ddots & \\
& & & A_{2}
\end{array}\right)
$$

is such that $f_{0} \circ A_{0}=3 \cdot f_{0}$. Computing the squares gives that both $\frac{1}{3} A_{1}^{2}$ and $\frac{1}{3} A_{2}^{2}$ have coefficients in $\mathbb{Z}$, and hence $\frac{1}{3} A_{0}^{2} \in \mathbf{O}_{f_{0}}(\mathbb{Z})$. Therefore by Lemma 4.18 , there exists a piece of the arithmetic manifold corresponding to $\mathbf{O}_{f}(\mathbb{Z})$ whose trace field increases to $\mathbb{Q}(\sqrt{3})$ under closing up. This procedure gives examples in any even dimension $n \geq 4$.

Table 4.1 below gives quadratic forms and matrices which can be used as in the example above to produce all quadratic extensions $\mathbb{Q}(\sqrt{d})$ for $d$ square-free, $2 \leq d \leq 42$. These examples were computed using the mathematical software Sage [Sage]. It follows from their construction that the volume of all these manifolds coincides with the volumes of the original arithmetic manifolds. 


\begin{tabular}{|c|c|c|c|}
\hline Field & Ouad & & \\
\hline$=\mathbb{Q}(\sqrt{2})$ & \rangle & $\left.\begin{array}{cccc}2 & 0 & 1 & 1 \\
1 & 0 & 1 & 1 \\
1 & 1 & 1 & 1 \\
1 & -1 & 1 & 1\end{array}\right)$ & $\left(\begin{array}{cc}1 & 1 \\
1 & -1\end{array}\right)$ \\
\hline$=\mathbb{Q}(\sqrt{3})$ & 11 & $\left(\begin{array}{cccc}2 & 0 & 1 & 0 \\
0 & 1 & 0 & 2 \\
-1 & 0 & -2 & 0 \\
0 & 1 & 0 & -1\end{array}\right)$ & $A_{2}=\left(\begin{array}{cc}1 & 2 \\
1 & -1\end{array}\right)$ \\
\hline$=\mathbb{Q}(\sqrt{5})$ & $\begin{array}{l}q_{1}= \\
q_{2}=\end{array}$ & $A_{1}=\left(\begin{array}{cccc}3 & 0 & 0 & 2 \\
0 & 1 & 2 & 0 \\
0 & 2 & -1 & 0 \\
-2 & 0 & 0 & -3\end{array}\right)$ & $A_{2}=\left(\begin{array}{cc}1 & 2 \\
2 & -1\end{array}\right)$ \\
\hline$k=\mathbb{Q}(\sqrt{6})$ & $\begin{array}{l}q_{1}=\langle- \\
q_{2}=\langle 1,\end{array}$ & $\left.\begin{array}{cccc}3 & 0 & 1 & 2 \\
0 & 0 & -2 & 2 \\
1 & 2 & 1 & 2 \\
1 & -1 & 1 & 2\end{array}\right)$ & $A_{2}=\left(\begin{array}{cc}2 & 2 \\
1 & -2\end{array}\right)$ \\
\hline$k=\mathbb{Q}(\sqrt{7})$ & $1,1,3\rangle$ & $\left(\begin{array}{cccc}3 & 1 & 1 & 0 \\
-1 & -1 & -2 & 3 \\
-1 & -2 & -1 & -3 \\
0 & 1 & -1 & -1\end{array}\right)$ & $A_{2}=\left(\begin{array}{cc}2 & 3 \\
1 & -2\end{array}\right)$ \\
\hline$k=\mathbb{Q}(\sqrt{10})$ & $\begin{array}{l}q_{1}= \\
q_{2}=\end{array}$ & $\left(\begin{array}{cccc}4 & 1 & 1 & 2 \\
1 & 3 & -1 & 1 \\
-1 & 1 & 1 & -3 \\
2 & 1 & 3 & 2\end{array}\right)$ & $A_{2}=\left(\begin{array}{cc}1 & 3 \\
3 & -1\end{array}\right)$ \\
\hline$k=\mathbb{Q}(\sqrt{11})$ & $\begin{array}{l}q_{1}= \\
q_{2}=\end{array}$ & $\left(\begin{array}{cccc}4 & 1 & 2 & 0 \\
-1 & 0 & -2 & 4 \\
-2 & -2 & -3 & -2 \\
0 & 2 & -1 & -1\end{array}\right)$ & $A_{2}=\left(\begin{array}{cc}3 & 2 \\
1 & -3\end{array}\right)$ \\
\hline$k=\mathbb{Q}(\sqrt{13})$ & $\begin{array}{l}q_{1}= \\
q_{2}=\end{array}$ & $\left(\begin{array}{cccc}4 & 1 & 1 & 1 \\
-1 & 1 & -2 & -3 \\
-1 & -2 & -3 & 1 \\
-1 & -3 & 1 & -2\end{array}\right)$ & $A_{2}=\left(\begin{array}{cc}2 & 3 \\
3 & -2\end{array}\right)$ \\
\hline$k=\mathbb{Q}(\sqrt{14})$ & $\begin{array}{l}q_{1}=\langle-1,1,1,5\rangle \\
q_{2}=\langle 1,5\rangle\end{array}$ & $A_{1}=\left(\begin{array}{cccc}4 & 1 & 1 & 0 \\
-1 & -1 & -3 & 5 \\
-1 & -3 & -1 & -5 \\
0 & 1 & -1 & -2\end{array}\right)$ & $A_{2}=\left(\begin{array}{cc}3 & 5 \\
1 & -3\end{array}\right)$ \\
\hline$c=\mathbb{Q}(\sqrt{15})$ & $\begin{array}{l}q_{1}=\langle-1,1,1,6\rangle \\
q_{2}=\langle 1,6\rangle\end{array}$ & $\left(\begin{array}{cccc}4 & 0 & 1 & 0 \\
0 & 3 & 0 & 6 \\
-1 & 0 & -4 & 0 \\
0 & 1 & 0 & -3\end{array}\right)$ & $A_{2}=\left(\begin{array}{cc}3 & 6 \\
1 & -3\end{array}\right)$ \\
\hline$k=\mathbb{Q}(\sqrt{17})$ & $\begin{array}{l}q_{1}= \\
q_{2}=\end{array}$ & $\left.\begin{array}{cccc}5 & 0 & 2 & 2 \\
0 & 3 & 2 & -2 \\
-2 & 2 & -4 & -1 \\
-2 & -2 & -1 & -4\end{array}\right)$ & $A_{2}=\left(\begin{array}{cc}1 & 4 \\
4 & -1\end{array}\right)$ \\
\hline$k=\mathbb{Q}(\sqrt{19})$ & $q_{2}=$ & $\left(\begin{array}{cccc}5 & 0 & 2 & 2 \\
0 & -4 & 1 & -2 \\
-2 & 1 & -2 & -6 \\
-1 & -1 & -3 & 1\end{array}\right)$ & $A_{2}=\left(\begin{array}{cc}1 & 6 \\
3 & -1\end{array}\right)$ \\
\hline$=\mathbb{Q}(\sqrt{21})$ & $\begin{array}{l}q_{1}=\langle-1,1,1,3\rangle \\
q_{2}=\langle 1,3\rangle\end{array}$ & $\left(\begin{array}{cccc}5 & 0 & 1 & 3 \\
0 & -3 & 3 & -3 \\
-1 & 3 & 1 & -6 \\
-1 & -1 & -2 & -3\end{array}\right)$ & $A_{2}=\left(\begin{array}{cc}3 & 6 \\
2 & -3\end{array}\right)$ \\
\hline$k=\mathbb{Q}(\sqrt{22})$ & $\begin{array}{l}q_{1}=\langle-1,1,1,2\rangle \\
q_{2}=\langle 1,2\rangle\end{array}$ & $\left(\begin{array}{cccc}5 & 0 & 1 & 2 \\
0 & -4 & 2 & -2 \\
-1 & 2 & 1 & -6 \\
-1 & -1 & -3 & -2\end{array}\right)$ & $A_{2}=\left(\begin{array}{cc}2 & 6 \\
3 & -2\end{array}\right)$ \\
\hline$k=\mathbb{Q}(\sqrt{23})$ & $\begin{array}{l}q_{1}=\langle-1,1,1,7\rangle \\
q_{2}=\langle 1,7\rangle\end{array}$ & $\left(\begin{array}{cccc}5 & 1 & 1 & 0 \\
-1 & -1 & -4 & 7 \\
-1 & -4 & -1 & -7 \\
0 & 1 & -1 & -3\end{array}\right)$ & $A_{2}=\left(\begin{array}{cc}4 & 7 \\
1 & -4\end{array}\right)$ \\
\hline$k=\mathbb{Q}(\sqrt{26})$ & $\begin{array}{l}q_{1}=\langle-1,1,1,1\rangle \\
q_{2}=\langle 1,1\rangle\end{array}$ & $\left(\begin{array}{cccc}6 & 0 & 1 & 3 \\
0 & -4 & 3 & -1 \\
-1 & 3 & 3 & -3\end{array}\right)$ & $A_{2}=\left(\begin{array}{cc}1 & 5 \\
5 & -1\end{array}\right)$ \\
\hline$k=\mathbb{Q}(\sqrt{29})$ & $\begin{array}{l}q_{1}=\langle-1,1,1,1\rangle \\
q_{2}=\langle 1,1\rangle\end{array}$ & $A_{1}=\left(\begin{array}{cccc}7 & 0 & 2 & 4 \\
0 & -3 & 4 & -2 \\
-2 & 4 & 1 & -4\end{array}\right)$ & $A_{2}=\left(\begin{array}{cc}2 & 5 \\
5 & -\end{array}\right.$ \\
\hline
\end{tabular}




$$
\begin{aligned}
& k=\mathbb{Q}(\sqrt{30}) \quad \begin{array}{l}
q_{1}=\langle-1,1,1,5\rangle \\
q_{2}=\langle 1,5\rangle
\end{array} \quad A_{1}=\left(\begin{array}{cccc}
6 & 0 & 1 & 5 \\
0 & 0 & 5 & -5 \\
-1 & 5 & -1 & -5 \\
-1 & -1 & -1 & -5
\end{array}\right) \quad A_{2}=\left(\begin{array}{cc}
5 & 5 \\
1 & -5
\end{array}\right) \\
& k=\mathbb{Q}(\sqrt{31}) \quad \begin{array}{l}
q_{1}=\langle-1,1,1,3\rangle \\
q_{2}=\langle 1,3\rangle
\end{array} \quad A_{1}=\left(\begin{array}{cccc}
6 & 1 & 1 & 3 \\
-1 & 1 & 2 & -9 \\
-1 & 2 & -5 & -3 \\
-1 & -3 & -1 & -2
\end{array}\right) \quad A_{2}=\left(\begin{array}{cc}
2 & 9 \\
3 & -2
\end{array}\right)
\end{aligned}
$$

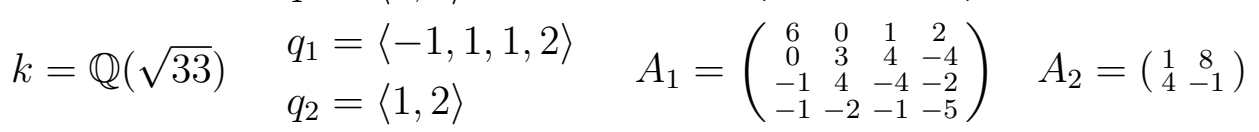

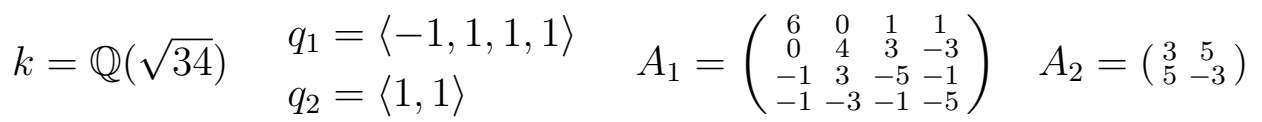

$$
\begin{aligned}
& k=\mathbb{Q}(\sqrt{35}) \quad \begin{array}{l}
q_{1}=\langle-1,1,2,5\rangle \\
q_{2}=\langle 2,5\rangle
\end{array} \quad A_{1}=\left(\begin{array}{cccc}
6 & 1 & 0 & 0 \\
-1 & -6 & 0 & 0 \\
0 & 0 & 5 & 5 \\
0 & 0 & 2 & -5
\end{array}\right) \quad A_{2}=\left(\begin{array}{cc}
5 & 5 \\
2 & -5
\end{array}\right) \\
& k=\mathbb{Q}(\sqrt{37}) \quad \begin{array}{l}
q_{1}=\langle-1,1,1,1\rangle \\
q_{2}=\langle 1,1\rangle
\end{array} \quad A_{1}=\left(\begin{array}{cccc}
7 & 2 & 2 & 2 \\
-2 & 1 & -2 & -6 \\
-2 & -2 & -6 & 1 \\
-2 & -6 & 1 & -2
\end{array}\right) \quad A_{2}=\left(\begin{array}{cc}
1 & 6 \\
6 & -1
\end{array}\right) \\
& k=\mathbb{Q}(\sqrt{38}) \quad \begin{array}{l}
q_{1}=\langle-1,1,1,2\rangle \\
q_{2}=\langle 1,2\rangle
\end{array} \quad A_{1}=\left(\begin{array}{cccc}
7 & 0 & 3 & 2 \\
0 & -4 & 2 & -6 \\
-3 & 2 & -5 & -6 \\
-1 & -3 & -3 & 2
\end{array}\right) \quad A_{2}=\left(\begin{array}{cc}
6 & 2 \\
1 & -6
\end{array}\right) \\
& k=\mathbb{Q}(\sqrt{39}) \quad \begin{array}{l}
q_{1}=\langle-1,1,1,3\rangle \\
q_{2}=\langle 1,3\rangle
\end{array} \quad A_{1}=\left(\begin{array}{cccc}
7 & 1 & 3 & 0 \\
-1 & 2 & -3 & 9 \\
-3 & -3 & -6 & -3 \\
0 & 3 & -1 & -3
\end{array}\right) \quad A_{2}=\left(\begin{array}{cc}
6 & 3 \\
1 & -6
\end{array}\right) \\
& k=\mathbb{Q}(\sqrt{41}) \quad \begin{array}{l}
q_{1}=\langle-1,1,1,1\rangle \\
q_{2}=\langle 1,1\rangle
\end{array} \quad A_{1}=\left(\begin{array}{cccc}
7 & 0 & 2 & 2 \\
0 & 3 & 4 & -4 \\
-2 & 4 & -5 & -2 \\
-2 & -4 & -2 & -5
\end{array}\right) \quad A_{2}=\left(\begin{array}{cc}
4 & 5 \\
5 & -4
\end{array}\right) \\
& k=\mathbb{Q}(\sqrt{42}) \quad \begin{array}{l}
q_{1} \\
q_{2}=\langle-1,1,1,6\rangle
\end{array} \quad A_{1}=\left(\begin{array}{cccc}
7 & 0 & 1 & 6 \\
0 & 0 & 6 & -6 \\
-1 & 6 & -1 & -6 \\
-1 & -1 & -1 & -6
\end{array}\right) \quad A_{2}=\left(\begin{array}{cc}
6 & 6 \\
1 & -6
\end{array}\right)
\end{aligned}
$$

Table 4.1: Quadratic forms and matrices producing nontrivial trace fields under closing up, as in Example 4.5.2. 


\section{Chapter $5 \mid$ Pseudo-arithmeticity and Volumes}

The goal of this final chapter is to present the notion of pseudo-arithmeticity and motivate it using the trace field computations from the previous sections. The content of this chapter is a more detailed version of a joint work with Vincent Emery [EM18]. In the first section, we start by introducing pseudoarithmetic manifolds and deduce from the results established in the previous chapters that all gluings of arithmetic pieces are pseudo-arithmetic. In Section 5.2 we give the necessary background in the various homology theories needed for Section 5.3, where we prove (under an additional assumption on the ambient group) that the volume of a pseudo-arithmetic manifold is always a rational linear combination of volumes of arithmetic manifolds (in all dimensions $n \geq 4$ ).

\section{$5.1 \mid$ Pseudo-arithmeticity and gluings}

This section introduces and motivates the notion of pseudo-arithmeticity of lattices (and manifolds).

5.1.1 Pseudo-arithmeticity. Let $\Gamma \subset \mathrm{PO}(n, 1)$ be an arithmetic lattice. Recall that by definition (see Definition 2.3.2), there exists a totally real number field $k \subset \mathbb{R}$ and an algebraic $k$-group $\mathbf{G}$ such that:

1. $\mathbf{G}$ is admissible, that is, $\mathbf{G}(\mathbb{R}) \cong \mathrm{PO}(n, 1)$ and for each non-trivial embedding $\sigma: k \hookrightarrow \mathbb{R}$, the conjugate group ${ }^{\sigma} \mathbf{G}(\mathbb{R})$ is compact. In other words, $\mathbf{G}\left(k \otimes_{\mathbb{Q}} \mathbb{R}\right) \cong \mathrm{PO}(n, 1) \times$ compact (and the factor $\mathrm{PO}(n, 1)$ comes from the trivial embedding $k \subset \mathbb{R})$.

2. $\Gamma$ is commensurable with the image of $\mathbf{G}\left(\mathcal{O}_{k}\right) \hookrightarrow \mathbf{G}(\mathbb{R}) \stackrel{\sim}{\longrightarrow} \mathrm{PO}(n, 1)$.

By Theorem 3.13, $k$ is the trace field of $\Gamma$ and $\mathbf{G}$ is its ambient group. This allows one to generalize the notion of arithmeticity to any lattice $\Gamma \subset \mathrm{PO}(n, 1)$, by requiring that its ambient group mimics that of an arithmetic lattice. A 
first idea is to relax the two hypotheses on $\Gamma, \mathbf{G}$ above by requiring that only the first be satisfied.

Definition 5.1.1. A lattice $\Gamma \subset \operatorname{PO}(n, 1)$ with trace field $k$ and ambient group $\mathbf{G}$ is quasi-arithmetic if $\mathbf{G}$ is admissible (over $k$ ). A hyperbolic manifold $M=\Gamma \backslash \mathbf{H}^{n}$ is quasi-arithmetic if $\Gamma$ is.

Remark 5.1.2. As seen previously, any isomorphism $\mathbf{G}(\mathbb{R}) \cong \operatorname{PO}(n, 1)$ is induced by an $\mathbb{R}$-isomorphism of algebraic groups $\mathbf{G} \cong \mathbf{P O}_{(n, 1)}$ (see Remark 2.3.3). Therefore if $\sigma: k \hookrightarrow \mathbb{C}$ is a complex embedding the group ${ }^{\sigma} \mathbf{G}$ is isomorphic to $\mathbf{P O}_{(n, 1), \mathbb{C}}$ and thus ${ }^{\sigma} \mathbf{G}(\mathbb{C})$ is non-compact. It follows that for an algebraic $k$-group $\mathbf{G}$ to be admissible, $k$ must be totally real.

Moreover, for any non-trivial extension $K / k$, the group $\mathbf{G}_{K}$ cannot be admissible (over $K$ ). If $K$ has a complex place, this is implied by the previous argument. If $K$ is totally real, each embedding $k \hookrightarrow \mathbb{R}$ extends to $[K: k]$ embeddings $K \hookrightarrow \mathbb{R}$, and thus

$$
\mathbf{G}_{K}\left(K \otimes_{\mathbb{Q}} \mathbb{R}\right) \cong \mathbf{G}(k \otimes \mathbb{R})^{[K: k]}
$$

since ${ }^{\tau} \mathbf{G}_{K}={ }^{\sigma} \mathbf{G}$ for any embedding $\tau: K \hookrightarrow \mathbb{R}$ extending $\sigma: k \hookrightarrow \mathbb{R}$. Hence having only one compact factor on the left implies $[K: k]=1$.

It follows from the remark that the trace field of a quasi-arithmetic lattice $\Gamma$ must be totally real. Comparing with the definition of arithmeticity, one sees that a lattice $\Gamma$ is quasi-arithmetic if instead of requiring $\Gamma$ to be commensurable with $\mathbf{G}\left(\mathcal{O}_{k}\right)$ for $\mathbf{G}$ admissible, one just asks for $\Gamma \subset \mathbf{G}(k)$.

There are examples of quasi-arithmetic nonarithmetic lattices in any dimension given by the construction of Belolipetsky and Thomson (see Section 4.2.2). However, in the context of gluings, it turns out that this definition is not general enough to encompass all arithmetic gluings (that is, elements of $\mathcal{A}^{g l}$, see Example 4.1.5). The reason is that the ambient group of a gluing coincides with the ambient group of any of the piece used in its construction (see Remark 4.3.3). Thus as soon as a gluing procedure increases the trace field, the ambient group cannot be admissible, by Remark 5.1.2. However, it turns out that the trace field of a gluing of arithmetic pieces is not arbitrary, as the next theorem shows.

Theorem 5.1. Let $M \in \mathcal{A}^{\text {gl }}$ be a gluing of arithmetic pieces. Then:

1. All its arithmetic pieces have the same trace field, call it $k$.

2. The trace field $K$ of $M$ is a multiquadratic extension of $k$, that is, $K=k\left(\sqrt{a_{1}}, \ldots, \sqrt{a_{r}}\right)$ for some $a_{i} \in k$.

3. The ambient group of $M$ is $\mathbf{G}_{K}$ where $\mathbf{G}$ is the ambient group of any of the pieces used in its construction. 
This is Theorem 2.7 in [EM18]. We will give a proof in the next section.

Remark 5.1.3. When the gluing isometries are specified, one can actually compute the trace field explicitly using Theorem 4.13.

This motivates taking the following weakening of quasi-arithmeticity as definition of pseudo-arithmeticity.

Definition 5.1.4. A lattice $\Gamma \subset \mathrm{PO}(n, 1)$ with trace field $K$ and ambient group $\mathbf{G}$ is pseudo-arithmetic if there exists a subfield $k \subset K$ such that:

1. $\mathbf{G} \cong \mathbf{G}_{0, K}$ for some $k$-group $\mathbf{G}_{0}$ that is admissible (over $k$ ),

2. the extension $K / k$ is multiquadratic.

A hyperbolic manifold $M=\Gamma \backslash \mathbf{H}^{n}$ is pseudo-arithmetic if $\Gamma$ is.

Therefore, pseudo-arithmetic lattices are those whose ambient group mimics the properties of the ambient group of a gluing. It turns out that, surprisingly, all known examples of hyperbolic Coxeter lattices in dimension $n \geq 4$ are pseudo-arithmetic (see [EM18, Section 4]). Therefore, all known constructions of hyperbolic lattices in dimension $n \geq 4$ lead to pseudo-arithmetic manifolds.

Pseudo-arithmeticity also has important implications for volumes:

Theorem 5.2. Let $M$ be a pseudo-arithmetic manifold with trace field $K$ (a multiquadratic extension of $k$ ). Assume its ambient group is of the form $\mathbf{P O}_{f, K}$, for some quadratic form $f$ defined over $k$. Then there exist arithmetic manifolds $M_{1}, \ldots, M_{N}$ and rationals $\beta_{1}, \ldots, \beta_{N} \in \mathbb{Q}$ such that

$$
\operatorname{Vol}(M)=\beta_{1} \operatorname{Vol}\left(M_{1}\right)+\cdots+\beta_{N} \operatorname{Vol}\left(M_{N}\right)
$$

The integer $N$ from the theorem corresponds to the degree of the multiquadratic extension $K / k$, and the arithmetic manifolds come from quadratic forms obtained via a substitution $x \mapsto \alpha_{i} x$ in one of the variables of $f$, where $\left\{\alpha_{i}\right\}_{i}$ is a basis of $K$ as a $k$-vector space. This theorem will be proven in Section 5.3.

Remark 5.1.5. In even dimension, the theorem follows directly from the (generalized) Gauss-Bonnet Theorem. Indeed, for any (complete) hyperbolic manifold of finite volume and dimension $n=2 m$ we have

$$
\operatorname{Vol}(M)=\frac{(-1)^{m} 4^{m} m !}{n !} \cdot \pi^{m} \cdot \chi(M)
$$

where $\chi(M)$ is the Euler characteristic of $M$. Therefore the set of volumes of these manifolds is in $\pi^{m} \cdot \mathbb{Q}$, and they are all $\mathbb{Q}$-linearly dependent. 
5.1.2 Gluing of arithmetic pieces. This short section is devoted to the:

Proof of Theorem 5.1. Part 1. follows from Corollary 3.14 and part 3. from Remark 4.3.3. The proof of part 2. uses a repeated application of Theorem 4.10. Indeed, by definition, there is a sequence $M_{0}, \ldots, M_{s}$ where $M_{0} \in \mathcal{A}$ is an arithmetic piece, $M_{s}=M$, and $M_{i+1}$ is constructed from $M_{i}$ by interbreeding $M_{i}$ with a piece in $\mathcal{A}$ or closing up $M_{i}$.

We will prove the theorem by induction on the number $s$ of gluing operations (either interbreeding or closing up). At the base case $s=0$, we have an arithmetic piece, so the theorem holds. For the inductive case, we will handle both interbreeding and closing up at once. If $M_{i+1}$ is an interbreeding of $M_{i}$, we set $A_{2} \in \mathcal{A}$ to be the arithmetic piece to which we glue $M_{i}$, and we set $A_{1}$ to be the arithmetic piece in $M_{i}$ containing the boundary hypersurface to which $A_{2}$ is glued. If $M_{i+1}$ is the closing up of $M_{i}$, we set $A_{1}, A_{2}$ to be the two arithmetic pieces containing the boundary hypersurfaces of $M_{i}$ at which the closing up occurs. Observe that we allow the case $A_{1}=A_{2}$. Let $k_{i}$ denote the trace field of $M_{i}$.

For $j=1,2$, let $\widetilde{A}_{j} \subset \mathbf{H}_{f_{j}}$ be a model for $A_{j}$ with monodromy representation $\Lambda_{j} \subset \mathbf{P O}_{f_{j}}(k)$. Using Lemma 4.8 , we find a model $\widetilde{M}_{i}$ for $M_{i}$ contained in $\mathbf{H}_{f_{1}}$ with monodromy representation $\Gamma \subset \mathbf{P O}_{f_{1}}\left(k_{i}\right)$ such that

$$
\Lambda_{1}=\operatorname{Stab}_{\Gamma}\left(\widetilde{A}_{1}\right)
$$

Now by Theorem 4.10 (or rather, by its proof), the $\widetilde{A}_{j}$ and $f_{j}$ can be chosen such that the gluing isometry $\varphi$ admits an extension $\phi: \mathbf{H}_{f_{1}} \rightarrow \mathbf{H}_{f_{2}}$, such that the induced isomorphism $\Phi: \mathbf{P O}_{f_{1}, \ell} \rightarrow \mathbf{P} \mathbf{O}_{f_{2}, \ell}$ is defined over a quadratic extension $\ell=k(\sqrt{a})$ of $k$. Therefore, the induced isomorphism $\Phi$ seen from the point of view of the ambient group of $M_{i}$ has $k_{i}(\sqrt{a})$ as a field of definition. Since $a \in k$, the resulting field is indeed a multiquadratic extension of $k$.

\section{$5.2 \mid$ Homology}

This section recalls the basic facts about homology needed to prove Theorem 5.2 and defines the relative homology of $\mathrm{PO}(n, 1)$ with respect to its action on the boundary $\partial \mathbf{H}^{n}$.

5.2.1 Classical homology. Here we regroup fundamentals of homology and cohomology of groups, and their singular counterparts. The main references are Weibel [We94] and Brown [Br82].

Definition 5.2.1. Let $G$ be a group. The group ring $\mathbb{Z} G$ is the ring of all formal sums $\sum_{g \in G} a_{g} g$ where $a_{g} \in \mathbb{Z}$ and almost all are zero. A $\mathbb{Z} G$-module is 
a module over the ring $\mathbb{Z} G$, that is, a $\mathbb{Z}$-module with a linear action of $G$. A $\mathbb{Z} G$-module $A$ is free if it is isomorphic to a direct sum of copies of $\mathbb{Z} G$. It is projective if there exists a $\mathbb{Z} G$-module $B$ such that $A \oplus B$ is free. A (chain) complex of $\mathbb{Z} G$-modules is a family of $\mathbb{Z} G$-modules $A=\left\{A_{m}\right\}_{m \in \mathbb{Z}}$ together with homomorphisms $d: A_{m} \rightarrow A_{m-1}$ such that $d \circ d=0$. It is exact or acyclic if $\operatorname{ker} d=\operatorname{im} d$. The homology of a complex $A$ is the family of $\mathbb{Z}$-modules $H_{m}(A)_{m \in \mathbb{Z}}$ where

$$
H_{m}(A)=\operatorname{ker}\left(A_{m} \rightarrow A_{m-1}\right) / \operatorname{im}\left(A_{m+1} \rightarrow A_{m}\right)
$$

A projective resolution $P \rightarrow A$ of a $\mathbb{Z} G$-module $A$ is an exact chain complex

$$
\cdots \rightarrow P_{m+1} \rightarrow P_{m} \rightarrow P_{m-1} \rightarrow \cdots \rightarrow P_{1} \rightarrow P_{0} \rightarrow A \rightarrow 0 \rightarrow 0 \rightarrow \cdots
$$

such that all $P_{m}$ are projective.

It follows from the universal property of projective modules that projective resolutions exist for any $\mathbb{Z} G$-module.

We are ready to define homology of groups. Let $G$ be a group and $A, B$ be $\mathbb{Z} G$-modules.

Definition 5.2.2. For each $m$, the $\mathbb{Z}$-module $\operatorname{Tor}_{m}^{\mathbb{Z} G}(A, B)$ is, equivalently:

1. The $m$-th homology of the complex $P \otimes_{\mathbb{Z} G} B$, where $P$ is a projective resolution of $A$.

2. The $m$-th homology of the complex $A \otimes_{\mathbb{Z} G} Q$, where $Q$ is a projective resolution of $B$.

One can show that $\operatorname{Tor}_{m}^{\mathbb{Z G}}(A, B)$ is well defined and independent (up to canonical isomorphism) of the chosen projective resolutions $P$ or $Q$.

Definition 5.2.3. The group homology $H_{m}(G, A)$ is defined as

$$
H_{m}(G, A)=\operatorname{Tor}_{m}^{\mathbb{Z} G}(\mathbb{Z}, A) .
$$

In the special case where $A=\mathbb{Z}$ we write $H_{m}(G)=H_{m}(G, \mathbb{Z})$.

Any short exact sequence $0 \rightarrow A \rightarrow B \rightarrow C \rightarrow 0$ induces a long exact sequence

$$
\cdots \rightarrow H_{m}(G, A) \rightarrow H_{m}(G, B) \rightarrow H_{m}(G, C) \stackrel{\delta}{\rightarrow} H_{m-1}(G, A) \rightarrow \cdots
$$

of the corresponding homology groups. The homomorphism $\delta$ is called the connecting homomorphism. 
5.2.2 Simplicial homology. In this section we briefly recall how to compute simplicial homology of a triangulated space.

Definition 5.2.4. A simplicial complex is a set $\mathcal{K}$ of simplices which intersect at faces and such that every face of a simplex in $\mathcal{K}$ is also in $\mathcal{K}$. For a topological space $X$, a triangulation is a simplicial complex $\mathcal{K}$ that is homeomorphic to $X$.

It is to be noted that every smooth manifold can be triangulated (see [Mu16]). If $\mathcal{K}$ is a simplicial complex, it defines a chain complex $C=C(\mathcal{K})$ as follows:

- $C_{m}$ is the free $\mathbb{Z}$-module on the set of oriented $m$-simplices in $\mathcal{K}$, quotiented by the submodule generated by elements of the form $\sigma+\sigma^{\prime}$, where $\sigma^{\prime}$ is the $m$-simplex $\sigma$ with reverse orientation.

- If $\left(v_{0}, \ldots, v_{m}\right)$ is an oriented $m$-simplex, then define

$$
d\left(v_{0}, \ldots, v_{m}\right)=\sum_{i=0}^{m}(-1)^{i}\left(v_{0}, \ldots, \widehat{v}_{i}, \ldots, v_{m}\right)
$$

where $\left(v_{0}, \ldots, \widehat{v}_{i}, \ldots, v_{m}\right)$ is the oriented simplex obtained by removing vertex $v_{i}$. The map $d$ then induces a homomorphism $d: C_{m} \rightarrow C_{m-1}$ such that $d^{2}=0$.

Definition 5.2.5. The homology $H_{m}(\mathcal{K})$ of a simplicial complex $\mathcal{K}$ is the homology of $C(\mathcal{K})$. The (simplicial) homology $H_{m}(X)$ of a topological space $X$ is the homology of a triangulation of $X$. For a $\mathbb{Z}$-module $R$, the homology $H_{m}(X ; R)$ of $X$ with coefficients in $R$ is the homology of the complex $C(\mathcal{K}) \otimes_{\mathbb{Z}}$ $R$.

It can be shown that simplicial homology is independent of the triangulation and isomorphic to the standard singular homology (see [Ha02, Theorem 2.27]).

5.2.3 Relative homology. In this section we set $G=\operatorname{PO}(n, 1)^{\circ}$ (the orientation preserving isometries of $\mathbf{H}^{n}$ ) and denote by $\Omega=\partial \mathbf{H}^{n}$ the geometric boundary of $\mathbf{H}^{n}$. The group $G$ acts naturally on the set $\Omega$, and the diagonal action induces an action of $G$ on $\Omega^{m}$. Let $\mathbb{Z} \Omega^{m}$ denote the set of formal sums $\sum_{x \in \Omega^{m}} a_{x} x$ with $a_{x} \in \mathbb{Z}$ and almost all are zero; it is naturally a $\mathbb{Z} G$-module.

Let $S \subset G$ be a subgroup of $G$. The inclusion $S \hookrightarrow G$ induces an inclusion of rings $\mathbb{Z} S \hookrightarrow \mathbb{Z} G$ which allows us to see $\mathbb{Z} \Omega^{m}$ as a $\mathbb{Z} S$-module. Let $J \Omega$ be the kernel of the augmentation map

$$
\begin{aligned}
\epsilon: \mathbb{Z} \Omega & \longrightarrow \mathbb{Z} \\
\sum_{x \in \Omega} a_{x} x & \longmapsto \sum_{x \in \Omega} a_{x} .
\end{aligned}
$$


Definition 5.2.6. The relative homology of $S \subset G$ (or homology of $S$ relative to its action on $\Omega$ ) is

$$
H_{m}(S, \Omega)=H_{m-1}(S, J \Omega)=\operatorname{Tor}_{m-1}^{\mathbb{Z} S}(\mathbb{Z}, J \Omega) .
$$

For a $\mathbb{Z}$-module $R$, the relative homology with coefficients $R$ of $S \subset G$ is

$$
H_{m}(S, \Omega ; R)=H_{m-1}\left(S, J \Omega \otimes_{\mathbb{Z}} R\right) .
$$

We will be mainly interested in the case where $S=G$ or $S$ is a lattice in $G$. Observe that the short exact sequence of $\mathbb{Z} S$-modules

$$
0 \rightarrow J \Omega \rightarrow \mathbb{Z} \Omega \rightarrow \mathbb{Z} \rightarrow 0
$$

induces a long exact sequence in homology with a connecting homomorphism

$$
\delta: H_{m}(S)=H_{m}(S, \mathbb{Z}) \rightarrow H_{m-1}(S, J \Omega)=H_{m}(S, \Omega) .
$$

If $S$ acts freely on $\Omega$ (that is, $s x=x$ implies $s=1$ for any $s \in S, x \in \Omega$ ), then for any point $x \in \Omega$ the $\mathbb{Z} S$-submodule $\mathbb{Z} S \cdot x$ of $\mathbb{Z} \Omega$ is isomorphic to $\mathbb{Z} S$ and thus free. It follows (since it is the direct sum of those modules) that $\mathbb{Z} \Omega$ is a free $\mathbb{Z} S$-module, and by looking at the long exact sequence above one sees that $\delta$ is an isomorphism in that case.

5.2.4 Relative homology of lattices. This section treats the special case where $S=\Gamma$ is a lattice in $G=\operatorname{PO}(n, 1)^{\circ}$. We will assume that $\Gamma$ is torsion-free. Let $M=\Gamma \backslash \mathbf{H}^{n}$ denote the corresponding (orientable) manifold.

Consider first the end-compactification $\widehat{M}$ of $M$, that is, the compact topological space obtained by adjoining one point to each cusp. Its universal cover $X$ consists of $\mathbf{H}^{n}$ together with the set $\mathcal{C} \subset \partial \mathbf{H}^{n}$ of points fixed by parabolic elements of $\Gamma$. If $M$ is compact, then $\widehat{M}=M$ and thus $H_{n}(\widehat{M}) \cong \mathbb{Z}$. If $M$ is non-compact, let $M_{0} \subset M$ be a (large enough) compact subset such that $A=M \backslash M_{0}$ is a disjoint union of cusps. Then the closure $\widehat{A}$ of $A$ in $\widehat{M}$ is contractible, and thus $H_{n}(\widehat{M}) \cong H_{n}(\widehat{M}, \widehat{A})$ (see [Ha02, Section 2.1]). By excision, the latter is isomorphic to $H_{n}\left(M_{0}, \partial M_{0}\right) \cong \mathbb{Z}$. Thus in both cases, we have that $H_{n}(\widehat{M})=\mathbb{Z}$.

Fix a triangulation of $\widehat{M}$ such that the cusp points are contained in its vertices (one can do this by adding one vertex for each cusp to a triangulation of $\left.M_{0}\right)$. Let $C(\widehat{M})$ be the corresponding chain complex of $\mathbb{Z}$-modules. By lifting the triangulation of $\widehat{M}$ to a triangulation of its universal cover $X$, we obtain a chain complex $C(X)$, invariant under the action of $\Gamma$. It is therefore a $\mathbb{Z} \Gamma$-module, and we have $C(X) \otimes_{\mathbb{Z} \Gamma} \mathbb{Z}=C(X)_{\mathbb{Z} \Gamma} \cong C(\widehat{M})$. This implies that $H_{n}(\widehat{M})=H_{n}\left(C(X) \otimes_{\mathbb{Z} \Gamma} \mathbb{Z}\right)$.

Proposition 5.3. We have an isomorphism $H_{n}(\Gamma, \Omega) \cong H_{n}\left(C(X) \otimes_{\mathbb{Z} \Gamma} \mathbb{Z}\right)$. Thus

$$
H_{n}(\Gamma, \Omega) \cong H_{n}(\widehat{M}) \cong \mathbb{Z}
$$

Proof. See for instance [Em17, Section 3]. 
5.2.5 Relative homology and volumes. We use the notation from the previous section. In particular, $G=\mathrm{PO}(n, 1)^{\circ}, \Omega=\partial \mathbf{H}^{n}$ and $\Gamma \subset G$ is a torsion-free lattice. We begin with a definition.

Definition 5.2.7. The fundamental class $[\Gamma] \in H_{n}(G, \Omega)$ is the image of the generator of $H_{n}(\Gamma, \Omega) \cong \mathbb{Z}$ corresponding to the positive orientation in $H_{n}(\widehat{M})$, under the natural map induced by the inclusion $\Gamma \hookrightarrow G$.

As the next theorem shows, this gives a homological way to compute the volume of the corresponding manifold which will be one of the main ingredients in the proof of Theorem 5.2.

Proposition 5.4. There exists a linear map $v: H_{n}(G, \Omega) \rightarrow \mathbb{R}$ such that for any torsion-free lattice $\Gamma \subset G$,

$$
v([\Gamma])=\operatorname{Vol}\left(\Gamma \backslash \mathbf{H}^{n}\right) .
$$

Proof. See [NY99, Section 3,4] and [Em17, Section 3]. The main idea of the proof is the following. Consider the complex $A=\left(\mathbb{Z} \Omega^{m}\right)_{m \in \mathbb{N}}$ where $A_{0}=\mathbb{Z}$ and the boundary maps are given by

$$
\begin{aligned}
d: \mathbb{Z} \Omega^{m+1} & \longrightarrow \mathbb{Z} \Omega^{m} \\
\left(x_{0}, \ldots, x_{m}\right) & \longmapsto \sum_{i=0}^{m}(-1)^{i}\left(x_{0}, \ldots, \widehat{x}_{i}, \ldots, x_{m}\right) .
\end{aligned}
$$

This complex is acyclic, and contracting the last maps $\mathbb{Z} \Omega^{2} \rightarrow \mathbb{Z} \Omega \rightarrow \mathbb{Z} \rightarrow 0$ into $\mathbb{Z} \Omega^{2} \rightarrow J \Omega \rightarrow 0$ gives an acyclic resolution of $J \Omega$. Let $B$ denote the contracted complex (so that $B_{0}=\mathbb{Z} \Omega^{2}$ and $B_{-1}=J \Omega$ ). The orientation of $\mathbf{H}^{n}$ induces an orientation on each ideal simplex. Hence we have a function $v: \Omega^{n+1} \rightarrow \mathbb{R}$ which assigns to a point $\left(x_{0}, \ldots, x_{n}\right)$ the (signed) volume of the oriented ideal simplex it defines. Since volume is invariant under (orientationpreserving) isometries, $v$ actually induces a map

$$
v:\left(\mathbb{Z} \Omega^{n+1}\right)_{\mathbb{Z} G}=\mathbb{Z} \Omega^{n+1} \otimes_{\mathbb{Z} G} \mathbb{Z} \longrightarrow \mathbb{R} .
$$

Moreover, it is not hard to see that it is zero on the image of $d$. Thus, $v$ induces a linear map

$$
v: H_{n-1}\left(B \otimes_{\mathbb{Z} G} \mathbb{Z}\right) \rightarrow \mathbb{R}
$$

Finally, let $P$ be a projective $\mathbb{Z} G$-resolution of $J \Omega$. Since the complex $B$ is acyclic, it follows from [Br82, Lemma I.74] that there exists a chain map $P \rightarrow B$ (unique up to homotopy) extending the identity $J \Omega \rightarrow J \Omega$. Thus, it induces a map in homology

$$
v: H_{n}(G, \Omega)=H_{n-1}\left(P \otimes_{\mathbb{Z} G} \mathbb{Z}\right) \longrightarrow H_{n-1}\left(B \otimes_{\mathbb{Z} G} \mathbb{Z}\right) \longrightarrow \mathbb{R} .
$$


By construction, the fundamental class $[\Gamma] \in H_{n}(G, \Omega)$ corresponds to a finite sum of oriented simplices in $H_{n}\left(C(X) \otimes_{\mathbb{Z} \Gamma} \mathbb{Z}\right)$. It turns out that modulo boundaries in $C(X)$, each of these simplices can be expressed as a sum of ideal simplices, whose signed volume equals that of the original simplex. The class in $H_{n}(G, \Omega)$ of this sum of oriented ideal simplices then coincides with $[\Gamma]$. Therefore by construction, $v([\Gamma])$ equals the (signed) volume of this sum, which equals the volume of the triangulation of $\widehat{M}$, i.e., $\operatorname{Vol}(M)$.

\subsection{Volumes of pseudo-arithmetic manifolds}

The goal of this section is to prove Theorem 5.2. We will start by computing the homology of the $k$-points $\mathbf{P} \mathbf{O}_{f}^{\circ}(k)$ of the algebraic groups $\mathbf{P} \mathbf{O}_{f}^{\circ}$, and deduce the theorem using results of [Em17].

5.3.1 Homology of ambient groups. Let $k \subset \mathbb{R}$ be a totally real number field, $K / k$ a totally real multiquadratic extension, and fix totally positive elements $a_{i} \in k$ such that $K=k\left(\sqrt{a_{1}}, \ldots, \sqrt{a_{r}}\right)$. Let $f_{0}=f_{0}\left(x_{0}, \ldots, x_{n-1}\right)$ be a quadratic form over $k$ that is admissible. Assume that $f_{0}$ is diagonal, with negative $x_{0}$ coefficient. Define the quadratic forms

$$
f_{i}=f_{0}+a_{i} x_{n}^{2}, \quad i \in\{0,1\}^{r}, \quad a_{i}=a_{1}^{i_{1}} \cdots a_{r}^{i_{r}} .
$$

Set $\alpha_{i}=\sqrt{a_{i}}$, so that the $\left\{\alpha_{i}\right\}$ form a basis of $K$ as $k$-vector space. Observe that

$$
f_{i}=f\left(x_{0}, \ldots, x_{n-1}, \alpha_{i} x_{n}\right), \quad \text { where } f=f_{0}+x_{n}^{2},
$$

so that the matrix

is such that $f_{i}=f \circ D_{i}$.

$$
D_{i}=\left(\begin{array}{cccc}
1 & & & \\
& \ddots & & \\
& & 1 & \\
& & & \alpha_{i}
\end{array}\right)
$$

Let $\mathbf{G}_{i}=\mathbf{P} \mathbf{O}_{f_{i}}^{\circ}$ and $\mathbf{G}=\mathbf{P} \mathbf{O}_{f, K}^{\circ}$ (the group $\mathbf{P} \mathbf{O}_{f}^{\circ}$ seen over $\left.K\right)$. It follows from the above definition that conjugation by $D_{i}$ induces a $K$-isomorphism of algebraic groups

$$
\varphi_{i}: \mathbf{G}_{i, K} \longrightarrow \mathbf{G} \text {. }
$$

In particular it induces an inclusion $\mathbf{G}_{i}(k) \hookrightarrow \mathbf{G}(K)$. The goal of this section is to prove Proposition 3.4 in [EM18]:

Proposition 5.5. The map induced by the inclusions $\mathbf{G}_{i}(k) \hookrightarrow \mathbf{G}(K)$

$$
\bigoplus_{i \in\{0,1\}^{r}} H_{n}\left(\mathbf{G}_{i}(k), \mathbb{Q}\right) \longrightarrow H_{n}(\mathbf{G}(K), \mathbb{Q})
$$

is surjective. 
5.3.2 Lemmas. Recall the following definition.

Definition 5.3.1. A morphism $\varphi: \mathbf{G}^{\prime} \rightarrow \mathbf{G}$ between two algebraic $k$-groups is an isogeny if it is surjective with finite kernel. An algebraic group $\mathbf{G}$ is simply connected if it is connected and if every isogeny $\mathbf{G}^{\prime} \rightarrow \mathbf{G}$ with $\mathbf{G}^{\prime}$ connected is an isomorphism.

Every semisimple connected algebraic group $\mathbf{G}$ has a simply connected cover, usually denoted $\widetilde{\mathbf{G}}$, which is unique up to isomorphism (see [PR94, Theorem 2.6]). Moreover, if $\mathbf{G}$ is adjoint, there is an exact sequence

$$
1 \rightarrow \mathbf{Z} \rightarrow \widetilde{\mathbf{G}} \rightarrow \mathbf{G} \rightarrow 1
$$

where $\mathbf{Z}$ denotes the center of $\widetilde{\mathbf{G}}$.

We will need three lemmas. The first one relates the homologies of a group and its simply-connected cover.

Lemma 5.6. Let $\mathbf{G}$ be a connected semi-simple adjoint algebraic group defined over a field $K$, and let $\widetilde{\mathbf{G}}$ denote its simply-connected cover. The natural map $\widetilde{\mathbf{G}}(K) \rightarrow \mathbf{G}(K)$ induces a surjective map (for any $m \geq 0$ ):

$$
H_{m}(\widetilde{\mathbf{G}}(K), \mathbb{Q}) \rightarrow H_{m}(\mathbf{G}(K), \mathbb{Q}) .
$$

Proof. The proof is a generalization of the proof of [Em17, Proposition 4.2]. Observe that the exact sequence (5.1) induces an exact sequence

$$
1 \rightarrow \mathbf{Z}(K) \rightarrow \widetilde{\mathbf{G}}(K) \rightarrow \mathbf{G}(K) \rightarrow H^{1}(K, \mathbf{Z}) \rightarrow H^{1}(K, \widetilde{\mathbf{G}})
$$

in Galois cohomology (see [Se68, Proposition 1, p. 133]). Contracting this exact sequence we obtain a short exact sequence

$$
1 \rightarrow \widetilde{\mathbf{G}}(K) / \mathbf{Z}(K) \rightarrow \mathbf{G}(K) \rightarrow A \rightarrow 1,
$$

where $A=\operatorname{ker}\left(H^{1}(K, \mathbf{Z}) \rightarrow H^{1}(K, \widetilde{\mathbf{G}})\right)$.

Consider the following composition of maps

$$
\begin{aligned}
H_{m}(\widetilde{\mathbf{G}}(K), \mathbb{Q}) \longrightarrow & H_{m}(\widetilde{\mathbf{G}}(K) / \mathbf{Z}(K), \mathbb{Q}) \\
\downarrow & H_{m}(\widetilde{\mathbf{G}}(K) / \mathbf{Z}(K), \mathbb{Q})_{A} \longrightarrow H_{m}(\mathbf{G}(K), \mathbb{Q})
\end{aligned}
$$

where the horizontal maps are induced by the quotient $\widetilde{\mathbf{G}}(K) \rightarrow \widetilde{\mathbf{G}}(K) / \mathbf{Z}(K)$ and the inclusion $\widetilde{\mathbf{G}}(K) / \mathbf{Z}(K) \hookrightarrow \mathbf{G}(K)$ coming from the above exact sequence, respectively. 
The horizontal maps in (5.3) coincide with the edge homomorphisms of the two Lyndon-Hochschild-Serre spectral sequences associated with (5.2) (see [We94, 6.8.2]):

$$
\begin{aligned}
E_{p q}^{2} & =H_{p}\left(A, H_{q}(\widetilde{\mathbf{G}}(K) / \mathbf{Z}(K), \mathbb{Q})\right) \Rightarrow H_{p+q}(\mathbf{G}(K), \mathbb{Q}), \text { and } \\
E_{p q}^{2} & =H_{p}\left(\widetilde{\mathbf{G}}(K) / \mathbf{Z}(K), H_{q}(\mathbf{Z}(K), \mathbb{Q})\right) \Rightarrow H_{p+q}(\widetilde{\mathbf{G}}(K), \mathbb{Q}),
\end{aligned}
$$

respectively. Since both sequences collapse at $E^{2}$, the horizontal maps of (5.3) are isomorphisms. The vertical map is obviously surjective, and it is not hard to see (looking at the definition of edge homomorphisms) that the composition of the three maps in (5.3) is induced by $\widetilde{\mathbf{G}}(K) \rightarrow \mathbf{G}(K)$.

The second lemma relates the continuous cohomology of a Lie group to the cohomology of the "compact dual" of its associated symmetric space. We first fix some notation.

Let $\mathbf{G}$ be a real semisimple algebraic group such that $\mathbf{G}(\mathbb{R})$ is connected as a Lie group. Let $K$ be a maximal compact Lie subgroup of $\mathbf{G}(\mathbb{R})$, and $H$ a maximal compact real Lie subgroup of $\mathbf{G}(\mathbb{C})$ which contains $K$. Then the space $H / K$ is a smooth manifold called the compact dual of the symmetric space $\mathbf{G}(\mathbb{R}) / K$. If $\mathbf{G}^{\prime}$ is another real semisimple algebraic group with $\mathbf{G}^{\prime}(\mathbb{R})$ connected, and if $K^{\prime}, H^{\prime}$ are defined similarly as for $\mathbf{G}$, then for any $\mathbb{R}$-morphism of algebraic groups $f: \mathbf{G} \rightarrow \mathbf{G}^{\prime}$ we have $f(K) \subset K^{\prime}$ and $f(H) \subset H^{\prime}$. Therefore, $f$ induces a smooth map

$$
f: H / K \longrightarrow H^{\prime} / K^{\prime}
$$

between the compact duals (which we still denote by $f$ ).

Finally, we will write $H^{m}$ for the cohomology of a topological space, and $H_{\mathrm{ct}}^{m}$ for the continuous cohomology, that is, the cohomology restricted to the continuous cocycles (see [BW00, IX.1.1]).

Lemma 5.7. Let $\mathbf{G}$ be a real semisimple algebraic group such that $\mathbf{G}(\mathbb{R})$ is connected as a Lie group. Let $K$ be a maximal compact Lie subgroup of $\mathbf{G}(\mathbb{R})$, and $H$ a maximal compact real Lie subgroup of $\mathbf{G}(\mathbb{C})$ which contains $K$. Then for any $m \geq 0$, there is an isomorphism:

$$
H_{\mathrm{ct}}^{m}(\mathbf{G}(\mathbb{R}), \mathbb{R}) \stackrel{\sim}{\longrightarrow} H^{m}(H / K, \mathbb{R}) .
$$

Moreover, this isomorphism is functorial in the following sense: if $\mathbf{G}^{\prime}$ is another real semisimple algebraic group with $\mathbf{G}(\mathbb{R})$ connected, $K^{\prime}, H^{\prime}$ defined as for $\mathbf{G}$ and $f: \mathbf{G} \rightarrow \mathbf{G}^{\prime}$ an $\mathbb{R}$-morphism of algebraic groups, then the following diagram commutes:

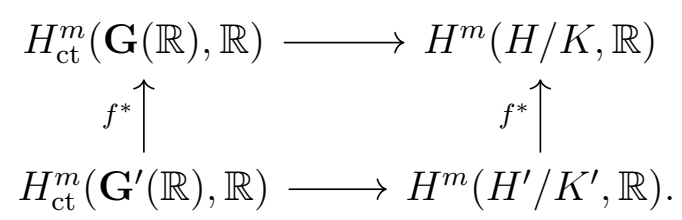


Proof. By [Bu02, Section 6.3], there are isomorphisms

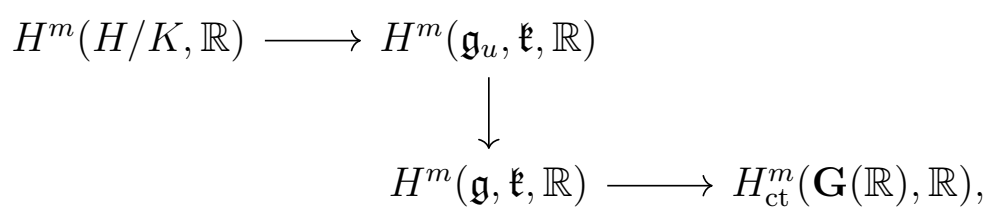

where:

- $\mathfrak{g}$ is the Lie algebra of $\mathbf{G}(\mathbb{R})$,

- $\mathfrak{k}$ is the Lie subalgebra of $\mathfrak{g}$ corresponding to $K$,

- $\mathfrak{g}_{u}$ is the Lie algebra of $H$.

Now it suffices to verify that all maps are functorial in the above sense. For both horizontal maps, this follows from the fact that the correspondence between a semisimple Lie group and its Lie algebra is a functor. Moreover, since the vertical map is induced by multiplication with a scalar on the level of Lie algebras, it is functorial as well (see the definition of $\iota$ in [Bu02, Section 6.3, Equation (6.2)]).

For the rest of this section, we will use the same notation as in the previous section. In particular, $k$ is a totally real number field, $K / k$ is a multiquadratic extension with $k$-basis $\alpha_{i}, i \in\{0,1\}^{r}, \mathbf{G}_{i}$ are algebraic groups corresponding to quadratic forms $f_{i}$ and $\varphi_{i}$ are the $K$-isomorphisms induced via conjugation by the diagonal matrices $D_{i}$ with entries $1, \ldots, 1, \alpha_{i}$.

The final lemma of this section studies the behaviour of the isomorphisms $\varphi_{i}$ on the compact duals corresponding to the groups $\mathbf{G}_{i}$. Recall that for $i \in\{0,1\}^{r}$, we assume that $f_{i}=f_{0}+a_{i} x_{n}^{2}$ is an admissible quadratic form with $f_{0}$ diagonal and negative $x_{0}$ coefficient. Let $\widehat{f}_{i}$ be the quadratic form $f_{i}$ with positive $x_{0}$ coefficient (the positive definite version of $f_{i}$ ), i.e.,

$$
\widehat{f_{i}}=f_{i}\left(\sqrt{-1} x_{0}, x_{1}, \ldots x_{n}\right) .
$$

Set $q_{i}=f_{i}\left(0, x_{1}, \ldots, x_{n}\right)$, and let $S_{i}=\mathbf{O}_{\widehat{f_{i}}}(\mathbb{R}) / \mathbf{O}_{q_{i}}(\mathbb{R})$, so that $S_{i}$ is homeomorphic to an $n$-sphere. Write $S=S_{(0, \ldots, 0)}$ and observe that

$$
\widehat{f}_{i}=\widehat{f}_{(0, \ldots, 0)} \circ D_{i}
$$

Hence, as before, conjugation by $D_{i}$ induces an isomorphism $\varphi_{i}: S_{i} \rightarrow S$ (which we still denote $\left.\varphi_{i}\right)$. Write $\operatorname{Gal}(K / k)=\left\{\sigma_{j}\right\}_{j \in\{0,1\}^{r}}$ and let $\left({ }^{\sigma_{j}} \varphi_{i}\right): S_{i} \rightarrow S$ be induced via conjugation by the diagonal matrix $\sigma_{j}\left(D_{i}\right)$. Finally, let $S^{\{0,1\}^{r}}$ denote the product $\prod_{\{0,1\}^{r}} S$ and observe that its homology is isomorphic to $\bigoplus_{\{0,1\}^{r}} H_{n}(S)$ by the Künneth formula. 
Lemma 5.8. The following map is injective:

$$
\begin{aligned}
\bigoplus_{i} H_{n}\left(S_{i}\right) & \longrightarrow H_{n}\left(S^{\{0,1\}^{r}}\right) \cong \bigoplus_{j} H_{n}(S) \\
x_{i} & \longmapsto\left(\left({ }^{\sigma_{j}} \varphi_{i}\right)\left(x_{i}\right)\right)_{j}
\end{aligned}
$$

where $i$ and $j$ range over $\{0,1\}^{r}$ and $x_{i}$ is a generator of $H_{n}\left(S_{i}\right)$. It is an isomorphism when the scalars are extended to $\mathbb{Q}($ or $\mathbb{R})$.

Proof. We have ${ }^{\sigma_{j}} \varphi_{i}=\eta_{j i} \circ \varphi_{i}$ where $\eta_{j i}$ is induced via conjugation by the diagonal matrix

$$
\left(\begin{array}{cccc}
1 & & & \\
& \ddots & & \\
& & 1 & \\
& & & \beta_{j i}
\end{array}\right) \text { where } \beta_{j i}=\sigma_{j}\left(\alpha_{i}\right) / \alpha_{i}
$$

Observe that since $\sigma_{j}\left(\alpha_{i}\right)= \pm \alpha_{i}$, we have $\beta_{j i} \in\{ \pm 1\}$. Let us identify $S_{i}$ with $S$ by means of $\varphi_{i}$. The $\eta_{j i}$ are automorphisms of $S$, so they induce maps $\eta_{j i, *}$ of degree 1 in homology. More precisely, if $H_{n}(S)$ is identified with $\mathbb{Z}$, then $\eta_{j i, *}$ sends 1 to $\beta_{j i} \in\{ \pm 1\}$. With our identifications, the map in the statement of the lemma is simply

$$
\begin{aligned}
& \mathbb{Z}^{2^{r}} \longrightarrow \mathbb{Z}^{2^{r}} \\
& x=\left(x_{i}\right)_{i} \longmapsto\left(\sum_{i} \eta_{j i, *}\left(x_{i}\right)\right)_{j}=B x
\end{aligned}
$$

where $B$ is the matrix $B=\left(\beta_{j i}\right)_{i j}$ (here again, $i$ and $j$ range over $\{0,1\}^{r}$ ).

We claim that $B$ is invertible. Indeed, let $C$ be obtained from $B$ by multiplying its $i$ 'th row with $\alpha_{i}$. Then $C$ is the matrix $\left(\sigma_{j}\left(\alpha_{i}\right)\right)_{i j}$. Hence by definition,

$$
\operatorname{det}(C)=\text { discriminant in } K / k \text { of the basis }\left\{\alpha_{i}\right\} \text {. }
$$

It follows that $\operatorname{det}(B) \neq 0$, and the proof is complete.

5.3.3 Proofs. In this section we will prove Proposition 5.5 and use it (as well as results from [Em17]) to deduce Theorem 5.2.

Proof of Proposition 5.5. The isomorphisms $\varphi_{i}: \mathbf{G}_{i, K} \rightarrow \mathbf{G}$ induce isomorphisms $\varphi_{i}: \widetilde{\mathbf{G}}_{i, K} \rightarrow \widetilde{\mathbf{G}}$ (that we also denote by $\varphi_{i}$ ) of the simply connected covers. These are actually coming from conjugation by the matrix $D_{i}$ on the level of Spin groups. Hence by Lemma 5.6, it is enough to prove the proposition with $\mathbf{G}_{i}$ and $\mathbf{G}$ replaced with their universal covers. Dualizing to cohomology and extending scalars to $\mathbb{R}$ implies that we only need to show that the map

$$
H^{n}(\widetilde{\mathbf{G}}(K), \mathbb{R}) \longrightarrow \bigoplus_{i} H^{n}\left(\widetilde{\mathbf{G}}_{i}(k), \mathbb{R}\right), \quad x \longmapsto\left(\varphi_{i}^{*}(x)\right)_{i}
$$


is injective, where $\varphi_{i}^{*}$ is induced by $\varphi_{i}: \widetilde{\mathbf{G}}_{i, K} \rightarrow \widetilde{\mathbf{G}}$. We will actually show that it is an isomorphism.

Consider the following diagram, where the horizontal maps are induced by $k \hookrightarrow k \otimes_{\mathbb{Q}} \mathbb{R}$ and $K \hookrightarrow K \otimes_{\mathbb{Q}} \mathbb{R}$, and where $\psi_{i}^{*}$ still has to be defined:

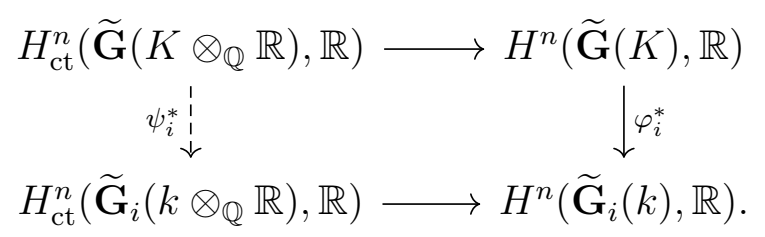

Since the $\widetilde{\mathbf{G}}_{i}$ and $\widetilde{\mathbf{G}}$ are simply-connected, their real points form a connected Lie group (see [Ma91, I.2.3, Remark 2]). By [Em17, Theorem 4.1] (see also the references therein) the horizontal maps are isomorphisms. Hence, to show that the map of (5.5) is an isomorphism, it is enough to construct $\psi_{i}^{*}$ such that the above diagram commutes and the following map is an isomorphism:

$$
H_{\mathrm{ct}}^{n}\left(\widetilde{\mathbf{G}}\left(K \otimes_{\mathbb{Q}} \mathbb{R}\right), \mathbb{R}\right) \longrightarrow \bigoplus_{i} H_{\mathrm{ct}}^{n}\left(\widetilde{\mathbf{G}}_{i}\left(k \otimes_{\mathbb{Q}} \mathbb{R}\right), \mathbb{R}\right), \quad x \longmapsto\left(\psi_{i}^{*}(x)\right)_{i}
$$

For each embedding $\tau: k \hookrightarrow \mathbb{R}$ let $\widehat{\tau}: K \hookrightarrow \mathbb{R}$ be a fixed embedding of $K$ extending $\tau$. Then there are isomorphisms

$$
\widetilde{\mathbf{G}}_{i}\left(k \otimes_{\mathbb{Q}} \mathbb{R}\right)=\prod_{\tau: k \hookrightarrow \mathbb{R}}{ }^{\tau} \widetilde{\mathbf{G}}_{i}(\mathbb{R}) \text { and } \widetilde{\mathbf{G}}\left(K \otimes_{\mathbb{Q}} \mathbb{R}\right)=\prod_{\substack{\tau: k \hookrightarrow \mathbb{R} \\ \sigma \in \operatorname{Gal}(K / k)}} \widehat{\tau} \circ \sigma \widetilde{\mathbf{G}}(\mathbb{R})
$$

and it is not hard to check that (5.6) commutes if $\psi_{i}^{*}$ is induced by the following map:

$$
\psi_{i}: \widetilde{\mathbf{G}}_{i}\left(k \otimes_{\mathbb{Q}} \mathbb{R}\right) \longrightarrow \widetilde{\mathbf{G}}\left(K \otimes_{\mathbb{Q}} \mathbb{R}\right), \quad x=\left(x_{\tau}\right)_{\tau} \longmapsto\left(\left({ }^{(\widehat{T} \circ \sigma} \varphi_{i}\right)\left(x_{\tau}\right)\right)_{\tau, \sigma}
$$

where we understand $\left(\widehat{\tau} \circ \sigma \varphi_{i}\right)$ to be induced via conjugation by the diagonal matrix with entries $1, \ldots, 1,(\widehat{\tau} \circ \sigma)\left(\alpha_{i}\right)$.

Now that we have reduced the problem to a statement involving continuous cohomology, the final step is to pass to the compact dual using Lemma 5.7. First note that the compact dual of a Lie group is invariant under isogeny (since we mod out by a maximal compact subgroup). Therefore, as ${ }^{\tau} \widetilde{\mathbf{G}}_{i}$ is the simply connected cover of ${ }^{\tau} \mathbf{G}_{i}$, which is itself isogenous to ${ }^{\tau} \mathbf{O}_{f_{i}}=\mathbf{O}_{\left(\tau f_{i}\right)}$, we see that the compact dual of ${ }^{\tau} \widetilde{\mathbf{G}}_{i}(\mathbb{R})$ is homeomorphic to $\mathbf{O}_{\tau} f_{i}(\mathbb{R})$ quotiented by a maximal compact subgroup. Since ${ }^{\tau} f_{i}$ is positive definite when $\tau \neq$ id (by admissibility of $\left.f_{0}\right), \mathbf{O}_{\tau_{f_{i}}}(\mathbb{R})$ is compact, and thus its compact dual is trivial in that case. Finally if $\tau=\mathrm{id}$, the compact dual of $\mathbf{O}_{\tau_{i}}(\mathbb{R})=\mathbf{O}_{f_{i}}(\mathbb{R})$ is the sphere $S_{i}$ defined just before Lemma 5.8.

From this it follows that the compact dual of $\widetilde{\mathbf{G}}_{i}\left(k \otimes_{\mathbb{Q}} \mathbb{R}\right)$ is $S_{i}$, and a similar argument shows that the compact dual of $\widetilde{\mathbf{G}}\left(K \otimes_{\mathbb{Q}} \mathbb{R}\right)$ is $S^{\{0,1\}^{r}}=\prod_{\{0,1\}^{r}} S$. 
We dualize again, and it is routine to verify (using the functoriality of the isomorphism in Lemma 5.7) that the map induced by the $\psi_{i}$ on the compact duals coincides with the map in homology described in Lemma 5.8, which is an isomorphism since we are working with coefficients in $\mathbb{R}$. This concludes the proof of Proposition 5.5.

Theorem 5.2 is now a consequence of Proposition 5.5 and results from [Em17].

Proof of Theorem 5.2. We can assume that $n$ is odd (see Remark 5.1.5), and that $M$ is orientable (since the conclusion of the theorem is invariant under commensurability). Let $\Omega=\partial \mathbf{H}^{n}$. Write $K=k\left(\sqrt{a_{1}}, \ldots, \sqrt{a_{r}}\right)$ for some totally positive elements $a_{i} \in k$. Let $\mathbf{G}=\mathbf{P O}_{f, K}^{\circ}$ be the ambient group of $M$ and $\Gamma \subset \mathbf{G}(K)$ a monodromy representation. For $i \in\{0,1\}^{r}$, let $\mathbf{G}_{i}$ be defined as in Section 5.3.1 and let $\Gamma_{i} \subset \mathbf{G}_{i}(k)$ be torsion-free arithmetic lattices commensurable to $\mathbf{G}_{i}\left(\mathcal{O}_{k}\right)$. The proof can be summarized in the following diagram (where the homologies are taken with coefficients in $\mathbb{Q}$ ):

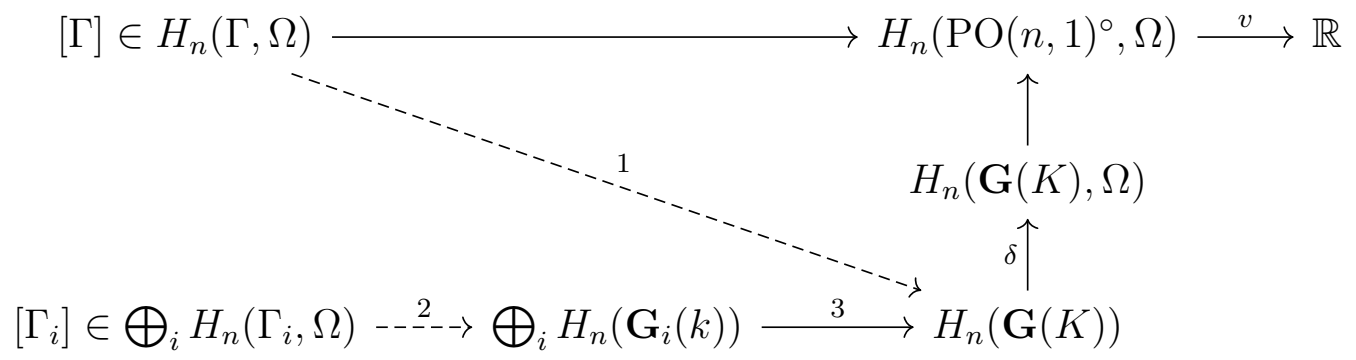

Here the unnamed maps are the natural ones, $\delta$ is the map defined in Section 5.2.3, $v$ comes from Section 5.2.5 and:

- The maps 1 and 2 exist when coefficients are extended to $\mathbb{Q}$ (this is Lemma 3.2 in [EM18]; see also [Em17, Proposition 6.1]).

- The map 2 is surjective (over $\mathbb{Q}): H_{n}\left(\mathbf{G}_{i}(k), \mathbb{Q}\right)$ has dimension one by [Em17, Proposition 4.2], and since $v\left(\left[\Gamma_{i}\right]\right) \neq 0$, the image of $\left[\Gamma_{i}\right]$ generates $H_{n}\left(\mathbf{G}_{i}(k), \mathbb{Q}\right)$.

- The map 3 is surjective (over $\mathbb{Q}$ ), by Proposition 5.5.

Since the top horizontal map factors through $H_{n}(\mathbf{G}(K)$ ), and since (over $\mathbb{Q})$ this space is generated by the images of the $\left[\Gamma_{i}\right]$, there are scalars $\beta_{i} \in \mathbb{Q}$, $i \in\{0,1\}^{r}$ such that:

$$
v([\Gamma])=\sum_{i} \beta_{i} v\left(\left[\Gamma_{i}\right]\right) .
$$

This completes the proof of Theorem 5.2. 


\section{Bibliography}

[Ag06] Ian Agol, Systoles of hyperbolic 4-manifolds, arXiv:math/0612290, 2006.

[BT11] Mikhail Belolipetsky and Scott Thomson, Systoles of hyperbolic manifolds, Algebr. Geom. Topol. 11 (2011), no. 3, 1455-1469.

[Be00] Nicolas Bergeron, Premier nombre de Betti et spectre du laplacien de certaines variétés hyperboliques, Enseign. Math. (2) 46 (2000), no. 1-2, 109-137.

[BG04] Nicolas Bergeron and Tsachik Gelander, A note on local rigidity, Geometriae Dedicata 107 (2004), no. 1, 111-131.

[BHW11] Nicolas Bergeron, Frédéric Haglund, and Daniel T. Wise, Hyperplane sections in arithmetic hyperbolic manifolds, J. Lond. Math. Soc. (2) 83 (2011), no. 2, 431-448.

[Bo69] Armand Borel, Introduction aux groupes arithmétiques, Actualités scientifiques et industrielles, vol. 1341, Hermann, 1969.

[Bo91] _ Linear algebraic groups (2nd enlarged ed.), Graduate texts in mathematics, vol. 126, Springer, 1991.

[BHC62] Armand Borel and Harish-Chandra, Arithmetic subgroups of algebraic groups, Ann. of Math. (2) 75 (1962), no. 3, 485-535.

[BP89] Armand Borel and Gopal Prasad, Finiteness theorems for discrete subgroups of bounded covolume in semi-simple groups, Inst. Hautes Études Sci. Publ. Math. (1989), no. 69, 119-171.

[BW00] Armand Borel and Nolan Wallach, Continuous cohomology, discrete subgroups, and representations of reductive groups, Math. Surveys Monogr., vol. 67, American Mathematical Society, 2000.

[Br10] Michel Brion, Introduction to actions of algebraic groups, Les cours du CIRM 1 (2010), no. 1, 1-22. 
[Br82] Kenneth S. Brown, Cohomology of groups, Graduate texts in mathematics, vol. 87, Springer, 1982.

[Bu02] José I. Burgos Gil, The regulators of Beilinson and Borel, CRM Monograph Series, vol. 15, American Mathematical Society, Providence, RI, 2002.

[Co14] Brian Conrad, Reductive group schemes, Autour des schémas en groupes. Vol. I, Panor. Synthèses, vol. 42/43, Soc. Math. France, Paris, 2014, pp. 93-444.

[CGP15] Brian Conrad, Ofer Gabber, and Gopal Prasad, Pseudo-reductive groups, 2 ed., New Mathematical Monographs, Cambridge University Press, 2015.

[DM86] Pierre Deligne and George D. Mostow, Monodromy of hypergeometric functions and non-lattice integral monodromy, Inst. Hautes Études Sci. Publ. Math. 63 (1986), 5-89.

[Di80] Jean Dieudonné, On the automorphisms of the classical groups, Memoirs of the American Mathematical Society, vol. 2, American Mathematical Society, Providence, R.I., 1980.

[Em17] Vincent Emery, On volumes of quasi-arithmetic hyperbolic lattices, Selecta Math. (N.S.) 23 (2017), 2849-2862.

[EM18] Vincent Emery and Olivier Mila, Hyperbolic manifolds and pseudoarithmeticity, arXiv:1810.12837, 2018.

[FLMS18] David Fisher, Jean-François Lafont, Nicholas Miller, and Matthew Stover, Finiteness of maximal geodesic submanifolds in hyperbolic hybrids, preprint arXiv:1802.04619v2, 2018.

[GL14] Tsachik Gelander and Arie Levit, Counting commensurability classes of hyperbolic manifolds, Geom. Funct. Anal. 24 (2014), no. 5, 1431-1447.

[GPS87] Mikhail Gromov and Ilya Piatetski-Shapiro, Nonarithmetic groups in Lobachevsky spaces, Inst. Hautes Études Sci. Publ. Math. (1987), no. $66,93-103$.

[EGA4] Alexander Grothendieck, Éléments de géométrie algébrique. IV. Étude locale des schémas et des morphismes de schémas. II, Inst. Hautes Études Sci. Publ. Math. (1965), no. 24, 231.

[Ha77] Robin Hartshorne, Algebraic geometry, Springer-Verlag, New YorkHeidelberg, 1977, Graduate Texts in Mathematics, No. 52. 
[Ha02] Allen Hatcher, Algebraic topology, Cambridge University Press, Cambridge, 2002.

[INV98] Max-Albert Knus, Alexander S. Merkurjev, Markus H. Rost, and Jean-Pierre Tignol, The book of involutions, AMS Colloquium Publications, vol. 44, American Mathematical Society, 1998.

[KRS18] Alexander Kolpakov, Alan W. Reid, and Leone Slavich, Embedding arithmetic hyperbolic manifolds, Math. Res. Lett. 25 (2018), no. 4, $1305-1328$.

[Ku85] Ernst Kunz, Introduction to commutative algebra and algebraic geometry, Birkhäuser Boston, Inc., Boston, MA, 1985.

[LM93] Jian-Shu Li and John J. Millson, On the first Betti number of a hyperbolic manifold with an arithmetic fundamental group, Duke Math. J. 71 (1993), no. 2, 365-401.

[MR03] Colin Maclachlan and Alan W. Reid, The arithmetic of hyperbolic 3-manifolds, Graduate texts in mathematics, vol. 219, Springer, 2003.

[Ma66] Vitalii S. Makarov, A certain class of discrete Lobacevskii space groups with an infinite fundamental region of finite measure, Soviet Mathematics, Doklady 167 (1966), no. 1, 328-331.

[Mu16] Ciprian Manolescu, Lectures on the triangulation conjecture, Proceedings of the Gökova Geometry-Topology Conference 2015, Gökova Geometry/Topology Conference (GGT), Gökova, 2016, pp. 1-38.

[Ma91] Grigori A. Margulis, Discrete subgroups of semisimple Lie groups, Ergeb. Math. Grenzgeb. (3), vol. 17, Springer, 1991.

[Ma16] Bruno Martelli, An introduction to geometric topology, CreateSpace Independent Publishing Platform, 2016.

[Me17] Jeffrey S. Meyer, Totally geodesic spectra of arithmetic hyperbolic spaces, Trans. Amer. Math. Soc. 369 (2017), no. 11, 7549-7588.

[Mi18] Olivier Mila, Nonarithmetic hyperbolic manifolds and trace rings, Algebr. Geom. Topol. 18 (2018), no. 7, 4359-4373.

[Mi13] James S. Milne, Lie Algebras, Algebraic Groups, and Lie Groups, 2013, Available at www.jmilne.org/math/.

[Mo15] Dave Witte Morris, Introduction to arithmetic groups, Deductive Press, 2015. 
[NY99] Walter Neumann and Jun Yang, Bloch invariants of hyperbolic 3manifolds, Duke Math. J. 96 (1999), no. 1, 29-59.

[NR91] Walter D. Neumann and Alan W. Reid, Amalgamation and the invariant trace field of a Kleinian group, Math. Proc. Cambridge Philos. Soc. 109 (1991), no. 3, 509-515. MR 1094749

[OV00] Arkadij L. Onishchik and Ernest B. Vinberg (eds.), Lie groups and Lie algebras II: I. Discrete subgroups of Lie groups, Encyclopaedia of mathematical sciences, vol. 21, Springer, 2000.

[PR94] Vladimir Platonov and Andrei S. Rapinchuck, Algebraic groups and number theory (engl. transl.), Pure and applied mathematics, vol. 139, Academic Press, 1994.

[PR09] Gopal Prasad and Andrei S. Rapinchuk, Weakly commensurable arithmetic groups and isospectral locally symmetric spaces, Publ. Math. Inst. Hautes Études Sci. 109 (2009), 113-184.

[Pr86] M. N. Prokhorov, Absence of discrete groups of reflections with a noncompact fundamental polyhedron of finite volume in a Lobachevskiu space of high dimension, Izv. Akad. Nauk SSSR Ser. Mat. 50 (1986), no. 2, 413-424.

[Ra72] M. S. Raghunathan, Discrete subgroups of Lie groups, SpringerVerlag, New York-Heidelberg, 1972, Ergebnisse der Mathematik und ihrer Grenzgebiete, Band 68.

[Ra13] Jean Raimbault, A note on maximal lattice growth in $\mathrm{SO}(1, n)$, Int. Math. Res. Not. IMRN (2013), no. 16, 3722-3731.

[Ra06] John G. Ratcliffe, Foundations of hyperbolic manifolds, second ed., Graduate Texts in Mathematics, vol. 149, Springer, New York, 2006.

[Se68] Jean-Pierre Serre, Corps locaux, Hermann, Paris, 1968, Deuxième édition, Publications de l'Université de Nancago, No. VIII.

[Se77] _ Cours d'arithmétique, Presses Universitaires de France, Paris, 1977, Deuxième édition revue et corrigée, Le Mathématicien, No. 2.

[Sage] The Sage Developers, Sagemath, the Sage Mathematics Software System (Version 8.6), 2019, http://www.sagemath.org.

[Th16] Scott Thomson, Quasi-arithmeticity of lattices in $\mathrm{PO}(n, 1)$, Geom. Dedicata 180 (2016), 85-94. 
[Ti66] Jacques Tits, Classification of algebraic semisimple groups, Proc. Sympos. Pure Math., vol. 9, 1966, pp. 33-62.

[Vi67] Ernest B. Vinberg, Discrete groups generated by reflections in Lobacevskii spaces, Sb. Math. 1 (1967), no. 3, 429-444.

[Vi71] _ Rings of definition of dense subgroups of semisimple linear groups, Math. USSR Izvestija 5 (1971), no. 1, 45-55.

[Vi81] _ The nonexistence of crystallographic reflection groups in Lobachevskiu spaces of large dimension, Funktsional. Anal. i Prilozhen. 15 (1981), no. 2, 67-68.

[Wa91] Bartel. L. van der Waerden, Algebra. Vol. II, Springer-Verlag, New York, 1991.

[We94] Charles A. Weibel, An introduction to homological algebra, Cambridge Studies in Advanced Mathematics, vol. 38, Cambridge University Press, Cambridge, 1994. 


\section{Erklärung}

gemäss Art. 30 RSL Phil.-nat. 18

Name / Vorname: Mila / Olivier

Matrikelnummer: 09-810-979

Studiengang: Mathematik, Dissertation

Titel der Arbeit: The trace field of hyperbolic gluings

Leiter der Arbeit: Prof. Dr. Vincent Emery

Ich erkläre hiermit, dass ich diese Arbeit selbständig verfasst und keine anderen als die angegebenen Quellen benutzt habe. Alle Stellen, die wörtlich oder sinngemäss aus Quellen entnommen wurden, habe ich als solche gekennzeichnet. Mir ist bekannt, dass andernfalls der Senat gemäss Artikel 28 RSL Phil.-nat. 05 zum Entzug des auf Grund dieser Arbeit verliehenen Titels berechtigt ist. Für die Zwecke der Begutachtung und der Überprüfung der Einhaltung der Selbständigkeitserklärung bzw. der Reglemente betreffend Plagiate erteile ich der Universität Bern das Recht, die dazu erforderlichen Personendaten zu bearbeiten und Nutzungshandlungen vorzunehmen, insbesondere die schriftliche Arbeit zu vervielfältigen und dauerhaft in einer Datenbank zu speichern sowie diese zur Überprüfung von Arbeiten Dritter zu verwenden oder hierzu zur Verfügung zu stellen.

Bern, 23. August 2019

Olivier Mila 


\title{
Lebenslauf
}

\author{
1991 Geboren in Zürich \\ 1997 - 2003 Primarschule in Castanet-Tolosan (Frankreich), Penthaz \\ und Denges \\ 2003 - 2006 Sekundarschule in Morges \\ 2006 - 2009 Gymnasium in Morges \\ 2009 - 2013 Bachelor B.Sc. in Communication Systems an der EPFL, \\ Lausanne \\ 2013 - 2015 Master M.Sc. in Mathematics an der EPFL, Lausanne \\ 2015 - 2019 Doktorat and der Universität Bern
}

